Supporting Information:

\title{
Shape-persistent $\pi$-conjugated macrocycles with aggregation-induced emission property: synthesis, mechanofluorochromism, and mercury(II) detection
}

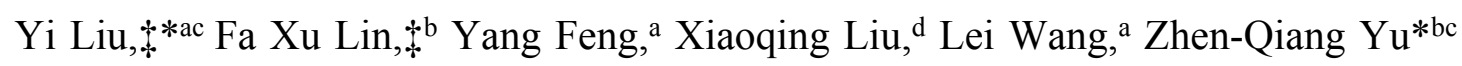
and Benzhong Tang*e

${ }^{a}$ Shenzhen Key Laboratory of Polymer Science and Technology, Guangdong Research Center for Interfacial Engineering of Functional Materials, College of Materials Science and Engineering, Shenzhen University, Shenzhen 518060, China.E-mail: liuyiacee@szu.edu.cn

${ }^{b}$ School of Chemistry and Environmental Engineering, Shenzhen University, Shenzhen 518060,

China.E-mail:zqyu@szu.edu.cn

${ }^{c}$ Centre for AIE Research, School of Material Science and Engineering, Shenzhen University, Shenzhen 518060, China.

${ }^{d}$ Shenzhen Grubbs Institute, Southern University of Science and Technology, Shenzhen 518005, China.

${ }^{e} H K U S T$ Shenzhen Research Institute, No. 9 Yuexing 1st RD, South Area, Hitech Park Nanshan, Shenzhen 518057, China.E-mail: tangbenz@ust.hk

$\$$ These authors contributed equally to this work. 


\section{Table of contents}

1. Synthetic procedures and structural characterization $S-3$

2. Figures and charts $\quad S-11$

3. NMR spectra of synthetic intermediates and products $\quad S-24$

4. Density functional theory (DFT) calculation $S-31$ 


\section{Synthetic procedures and structural characterization}

\section{4,4'-(2,2-bis(4-bromophenyl)ethene-1,1-diyl)bis(methoxybenzene) (3)}

A two-necked $250 \mathrm{~mL}$ round-bottom flask containing 4,4'-dibromobenzophenone (1.0 eq, $7.02 \mathrm{~g}$, 20.6mmol), 4,4'-dimethoxybenzophenone (1.0 eq, $5.00 \mathrm{~g}, 20.6 \mathrm{mmol})$, and Zinc dust (10.0 eq, $13.49 \mathrm{~g}, 206 \mathrm{mmol}$ ) was evacuated and refilled with argon gas for three times. Then, $80 \mathrm{~mL}$ of anhydrous THF was added into the flask, and the mixture was cooled down to $0^{\circ} \mathrm{C}$ in ice bath. TiCl4 (5.0 eq, $13.3 \mathrm{ml}, 103 \mathrm{mmol})$ was added into the mixture drop-wise, and the reaction mixture was then heated to $80^{\circ} \mathrm{C}$ and kept refluxing with stirring overnight. After evaporating the solvent, the crude product was purified via silica gel column chromatography with $\mathrm{DCM} / \mathrm{hexane}=1 / 6$ as eluent to obtain $4.50 \mathrm{~g}$ of the title compound as a light yellow solid (39.6\% yield). ${ }^{1} \mathrm{H}$ NMR (400 MHz, $\left.\mathrm{CDCl}_{3}\right) \delta / \mathrm{ppm}: 7.22(\mathrm{~d}, 4 \mathrm{H}, J=8.4 \mathrm{~Hz}), 6.90(\mathrm{~d}, 4 \mathrm{H}, J=8.5 \mathrm{~Hz}), 6.86(\mathrm{~d}, 4 \mathrm{H}, J=8.5 \mathrm{~Hz})$, $6.65(\mathrm{~d}, 4 \mathrm{H}, J=8.7 \mathrm{~Hz}), 3.74(\mathrm{~s}, 6 \mathrm{H}) .{ }^{13} \mathrm{C}$ NMR $\left(150 \mathrm{MHz}, \mathrm{CDCl}_{3}\right) \delta / \mathrm{ppm}: 158.26,143.93$, $142.87,137.10,136.14,132.63,131.99,130.19,130.00,126.31,125.44,122.86,113.13,55.12$.

\section{4',4'"-(2,2-bis(4-methoxyphenyl)ethene-1,1-diyl)bis(3-bromo-1,1'-biphenyl) (4)}

A two-necked $250 \mathrm{~mL}$ round-bottom flask containing compound 3 (1.0eq, $3.00 \mathrm{~g}, 5.45 \mathrm{mmol}$ ), bis(pinacolato)diboron (6.0eq, 8.31g, $32.7 \mathrm{mmol}), \mathrm{KAc}(6.0 \mathrm{eq}, 3.21 \mathrm{~g}, 32.7 \mathrm{mmol}), \mathrm{Pd}(\mathrm{dppf}) \mathrm{Cl}_{2}$ (0.1 eq, $398.9 \mathrm{mg}, 0.545 \mathrm{mmol})$, and 1,1'-bis(diphenylphosphino)ferrocene (0.1eq, $302.2 \mathrm{mg}, 0.55$ mmol) was evacuated and refilled with argon gas for three times. Then, $100 \mathrm{~mL}$ of anhydrous dioxane was added into the flask, and the mixture was then heated to $100{ }^{\circ} \mathrm{C}$ and kept refluxing with stirring overnight. After cooling to room temperature, the reaction mixture was extracted with ethyl acetate $(3 \times 100 \mathrm{~mL})$, and the combined organic layers were dried in vacuo. The crude product was then transferred to a two-necked $250 \mathrm{~mL}$ round-bottom flask, into which $\mathrm{K}_{2} \mathrm{CO}_{3}$ (6.0eq, 3.73g, 27.0mmol), 3-bromoiodobenzene (6.0eq, 3.47ml, $27.0 \mathrm{mmol})$, and $\mathrm{Pd}\left(\mathrm{PPh}_{3}\right)_{4}$ (0.06eq, $312 \mathrm{mg}, 0.27 \mathrm{mmol})$ were then added. The flask was evacuated and refilled with argon gas for three times, followed by the addition of degassed THF/EtOH/ $\mathrm{H}_{2} \mathrm{O}$ mixture $(80 \mathrm{~mL} / 10 \mathrm{~mL} / 10$ $\mathrm{mL}$ ). The mixture was then heated to $80{ }^{\circ} \mathrm{C}$ and kept refluxing with stirring overnight. After cooling to room temperature, the reaction mixture was extracted with dichloromethane $(3 \times 100$ $\mathrm{mL}$ ), and the combined organic layers were dried in vacuo. After evaporating the solvent, the crude product was purified via silica gel column chromatography with DCM/petroleum ether = 
$1 / 2.5$ as eluent to obtain $2.0 \mathrm{~g}$ of the title compound as a white solid (63.3\% yield). ${ }^{1} \mathrm{H}$ NMR (400

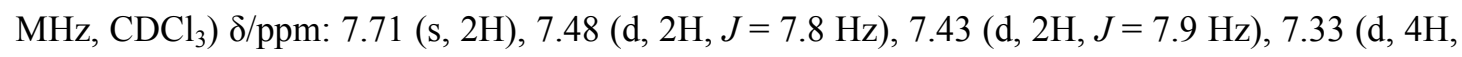
$J=8.4 \mathrm{~Hz}), 7.26(\mathrm{t}, 2 \mathrm{H}, J=7.9 \mathrm{~Hz}), 7.11(\mathrm{~d}, 4 \mathrm{H}, J=8.3 \mathrm{~Hz}), 6.98(\mathrm{~d}, 4 \mathrm{H}, J=8.7 \mathrm{~Hz}), 6.66(\mathrm{~d}$, $4 \mathrm{H}, J=8.6 \mathrm{~Hz}), 3.75(\mathrm{~s}, 6 \mathrm{H}) .{ }^{13} \mathrm{C} \mathrm{NMR}\left(150 \mathrm{MHz}, \mathrm{CDCl}_{3}\right) \delta / \mathrm{ppm}: 157.23,142.91,141.84$, $140.03,136.83,136.07,135.11,131.60,130.96,129.16,128.97,128.89,125.29,124.41,121.83$, 112.11, 54.09.

\section{4',4"'-(2,2-bis(4-(hexyloxy)phenyl)ethene-1,1-diyl)bis(3-bromo-1,1'-biphenyl) (5)}

A two-necked $250 \mathrm{~mL}$ round-bottom flask containing compound $\mathbf{4}(1.0 \mathrm{eq}, 1.00 \mathrm{~g}, 1.42 \mathrm{mmol})$ was evacuated and refilled with argon gas for three times. Then, $40 \mathrm{~mL}$ of anhydrous dichloromethane was added into the flask, and the mixture was cooled down to $0^{\circ} \mathrm{C}$ in ice bath. $\mathrm{BBr}_{3}$ (3.0 eq, 0.7 $\mathrm{ml}, 7.12 \mathrm{mmol}$ ) was added into the mixture drop-wise, and the reaction mixture was recovered to room temperature and kept stirring overnight. The reaction mixture was extracted with dichloromethane $(3 \times 40 \mathrm{~mL})$, and the combined organic layers were dried in vacuo. The crude product was then dissolved in acetone in a $250 \mathrm{~mL}$ round-bottom flask, in which $\mathrm{C}_{6} \mathrm{H}_{13} \mathrm{Br}$ (6.0 eq, $1.2 \mathrm{ml}, 8.54 \mathrm{mmol})$, and $\mathrm{Cs}_{2} \mathrm{CO}_{3}(4.0 \mathrm{eq}, 1.86 \mathrm{~g}, 5.69 \mathrm{mmol})$ was added into the mixture. The mixture was then heated to $80^{\circ} \mathrm{C}$ and kept refluxing with stirring overnight. After evaporating the solvent, the crude product was purified via silica gel column chromatography with $\mathrm{DCM} /$ petroleum ether $=1 / 5$ as eluent to obtain $1.12 \mathrm{~g}$ of the title compound as a light yellow solid (93.4\% yield). ${ }^{1} \mathrm{H}$ NMR (400 MHz, $\mathrm{CDCl}_{3}$ ) $\delta / \mathrm{ppm}: 7.68$ (s, 2H), 7.45 (d, 2H, J = $7.8 \mathrm{~Hz}$ ), 7.40 (d, $2 \mathrm{H}, \mathrm{J}=8.0 \mathrm{~Hz}), 7.30(\mathrm{~d}, 4 \mathrm{H}, J=8.3 \mathrm{~Hz}), 7.20(\mathrm{t}, 2 \mathrm{H}, J=7.7 \mathrm{~Hz}), 7.10(\mathrm{~d}, 4 \mathrm{H}, J=8.3 \mathrm{~Hz}), 6.96$ (d, 4H, $J=8.7 \mathrm{~Hz}), 6.64$ (d, 4H, $J=8.7 \mathrm{~Hz}), 3.86$ (t, 4H, $J=6.6 \mathrm{~Hz}), 1.73(\mathrm{~m}, 4 \mathrm{H}), 1.43(\mathrm{~m}, 4 \mathrm{H})$, $1.31(\mathrm{~m}, 8 \mathrm{H}), 0.89$ (d, 6H, $J=6.6 \mathrm{~Hz}) .{ }^{13} \mathrm{C}$ NMR (150 MHz, $\left.\mathrm{CDCl}_{3}\right) \delta / \mathrm{ppm}: 157.85,144.03$, $142.87,141.24,137.60,137.00,135.91,132.61,132.00,130.16,129.89,126.28,125.41,122.84$, $113.62,67.83,31.61,29.26,25.73,22.59,14.03$.

(((2,2-bis(4-(hexyloxy)phenyl)ethene-1,1-diyl)bis([1,1'-biphenyl]-4',3-diyl))bis(ethyne-2,1-diyl ))bis(trimethylsilane) (6)

A two-necked $250 \mathrm{~mL}$ round-bottom flask containing compound 5 (1.0 eq, $4.50 \mathrm{~g}, 5.37 \mathrm{mmol})$, $\mathrm{CuI}(0.1$ eq, $102 \mathrm{mg}, 0.54 \mathrm{mmol}), \operatorname{Pd}\left(\mathrm{PPh}_{3}\right)_{4}(0.1 \mathrm{eq}, 620 \mathrm{mg}, 0.54 \mathrm{mmol})$, and $\mathrm{PPh}_{3}(0.2$ eq, 281 $\mathrm{mg}, 1.07 \mathrm{mmol}$ )was evacuated and refilled with argon gas for three times. Then, diisopropylamine 
$(1 \mathrm{~mL})$, trimethylsilylacetylene $(6.0 \mathrm{eq}, 4.6 \mathrm{~mL}, 32 \mathrm{mmol})$ and $80 \mathrm{~mL}$ of anhydrous toluene was added into the flask, and the mixture was then heated to $120{ }^{\circ} \mathrm{C}$ and kept refluxing with stirring overnight. After evaporating the solvent, the crude product was purified via silica gel column chromatography with $\mathrm{DCM} /$ petroleum ether $=1 / 5$ as eluent to obtain $3.6 \mathrm{~g}$ of the title compound as yellow green solid (76.8\% yield). ${ }^{1} \mathrm{H}$ NMR $\left(400 \mathrm{MHz}, \mathrm{CDCl}_{3}\right) \delta 7.68(\mathrm{~s}, 2 \mathrm{H}), 7.51(\mathrm{~d}, 2 \mathrm{H}, J=$ $7.9 \mathrm{~Hz}$ ), 7.40 (d, 2H, $J=7.7 \mathrm{~Hz}), 7.37-7.30$ (m, 6H), 7.11 (d, 4H, $J=8.3 \mathrm{~Hz}), 6.96$ (d, 4H, $J=8.5$ $\mathrm{Hz}), 6.65(\mathrm{~d}, 4 \mathrm{H}, J=8.7 \mathrm{~Hz}), 3.88(\mathrm{t}, 4 \mathrm{H}, J=6.6 \mathrm{~Hz}), 1.74(\mathrm{~m}, 4 \mathrm{H}), 1.43(\mathrm{~m}, 4 \mathrm{H}), 1.36-1.30(\mathrm{~m}$, $8 \mathrm{H}), 0.89$ (t, 6H, J = 6.7 Hz), 0.26 (s, 18H). $\left.{ }^{13} \mathrm{C} \mathrm{NMR} \mathrm{(150} \mathrm{MHz,} \mathrm{CDCl}_{3}\right) \delta / \mathrm{ppm}: 157.81,143.78$, $140.94,140.83,137.88,137.60,136.07,132.64,131.94,131.89,130.51,130.49,128.59,127.03$, $126.83,126.34,126.27,126.22,123.45,113.62,105.12,94.18,67.85,67.81,31.64,31.61,29.30$, $25.76,22.61,14.06,2.001$.

\section{4',4'"-(2,2-bis(4-(hexyloxy)phenyl)ethene-1,1-diyl)bis(3-ethynyl-1,1'-biphenyl) (7)}

A two-necked $250 \mathrm{~mL}$ round-bottom flask containing compound 6 (1.0 eq, $2.4 \mathrm{~g}, 2.74 \mathrm{mmol})$, $\mathrm{CuI}(0.1 \mathrm{eq}, 102 \mathrm{mg}, 0.54 \mathrm{mmol})$ was evacuated and refilled with argon gas for three times. Then, $80 \mathrm{~mL}$ of anhydrous THF was added into the flask followed by the addition of the solution (1.0 M in THF) of TBAF (3.0eq, $8.2 \mathrm{~mL}, 8.21 \mathrm{mmol}$ ). The mixture was then kept stirring for 2 hours. After quenching with water, the reaction mixture was extracted with dichloromethane $(3 \times 40 \mathrm{~mL})$, and the combined organic layers were dried in vacuo. The crude product was purified via silica gel column chromatography with DCM/petroleum ether $=2 / 5$ as eluent to obtain $0.94 \mathrm{~g}$ of the title compound as yellow green solid (47.0\% yield). ${ }^{1} \mathrm{H}$ NMR (400 MHz, $\mathrm{CDCl}_{3}$ ) $\delta / \mathrm{ppm}: 7.70$ (s, 2H), $7.55(\mathrm{~d}, 2 \mathrm{H}, J=8.1 \mathrm{~Hz}), 7.43(\mathrm{~d}, 2 \mathrm{H}, \mathrm{J}=7.9 \mathrm{~Hz}), 7.38-7.33(\mathrm{~m}, 6 \mathrm{H}), 7.12(\mathrm{~d}, 4 \mathrm{H}, \mathrm{J}=8.2 \mathrm{~Hz})$, $6.96(\mathrm{~d}, 4 \mathrm{H}, \mathrm{J}=8.5 \mathrm{~Hz}), 6.64(\mathrm{~d}, \mathrm{~J}=8.5 \mathrm{~Hz}, 4 \mathrm{H}), 3.88$ (t, 4H, J = $6.3 \mathrm{~Hz}), 3.08$ (s, 2H), 1.74 (q, 4H, J = 7.2 Hz), 1.42 (m, 4H),1.36-1.27 (m, 8H), 0.89 (t, 6H, J = 6.7 Hz). ${ }^{13} \mathrm{C}$ NMR (150 MHz, $\mathrm{CDCl}_{3}$ ) $\delta /$ ppm: 157.82, 143.85, 140.94, 141.06, 137.46, 136.01, 132.63, 131.98, 130.66, 130.60, $128.70,127.33,126.25,122.44,113.62,83.67,67.98,67.84,31.62,29.28,26.92,25.74,22.60$, 14.04 .

\section{Macrocyclic luminogen TPEMC}

Into a $1000 \mathrm{~mL}$ round-bottom flask was added compound $7(1.0 \mathrm{eq}, 400 \mathrm{mg}, 0.545 \mathrm{mmol})$, CuI (0.03eq, 3.1mg, 0.016mmol), $\mathrm{Pd}\left(\mathrm{PPh}_{3}\right) \mathrm{Cl}_{2}(0.025 \mathrm{eq}, 9.6 \mathrm{mg}, 0.014 \mathrm{mmol}), 300 \mathrm{~mL}$ of THF, and 
$300 \mathrm{~mL}$ of triethylamine. The mixture was kept stirring under room temperature for 7 days. After evaporating the solvent, the crude product was purified via silica gel column chromatography with $\mathrm{DCM} /$ petroleum ether $=1 / 5$ as eluent to obtain $150 \mathrm{mg}$ of the title compound as white solid $(32.1 \%$ yield). HRMS (MALDI-TOF, DCTB as matrix) m/z: [M]+calcd. for $\mathrm{C}_{108} \mathrm{H}_{100} \mathrm{O}_{4}$ : 1461.7655. Found: $1461.7659 .{ }^{1} \mathrm{H}$ NMR (600 MHz, $\left.\mathrm{CDCl}_{3}\right) \delta / \mathrm{ppm}: 7.74(\mathrm{~s}, 4 \mathrm{H}), 7.53(\mathrm{~d}, 4 \mathrm{H}, J=7.8 \mathrm{~Hz})$, $7.48(\mathrm{~d}, 4 \mathrm{H}, J=7.7 \mathrm{~Hz}), 7.37$ (t, 4H, $J=7.6 \mathrm{~Hz}), 7.33(\mathrm{~d}, 8 \mathrm{H}, J=8.4 \mathrm{~Hz}), 7.12(\mathrm{~d}, 8 \mathrm{H}, J=8.1 \mathrm{~Hz})$, $6.98(\mathrm{~d}, 8 \mathrm{H}, J=8.7 \mathrm{~Hz}), 6.67(\mathrm{~d}, 8 \mathrm{H}, J=8.7 \mathrm{~Hz}), 3.91(\mathrm{t}, 8 \mathrm{H}, J=6.7 \mathrm{~Hz}), 1.76(\mathrm{q}, 8 \mathrm{H}, J=7.2 \mathrm{~Hz})$, $1.45(\mathrm{~m}, 8 \mathrm{H}), 1.34(\mathrm{~m}, 16 \mathrm{H}), 0.91(\mathrm{t}, 12 \mathrm{H}, J=6.7 \mathrm{~Hz}) .{ }^{13} \mathrm{C}$ NMR $\left(150 \mathrm{MHz}, \mathrm{CDCl}_{3}\right) \delta / \mathrm{ppm}$ : $157.85,143.85,141.32,137.53,136.20,132.72,132.13,131.14,130.95,128.78,128.03,126.38$ $122.12,113.67,81.54,73.91,67.89,31.65,29.72,29.31,25.78,22.63,14.07$.

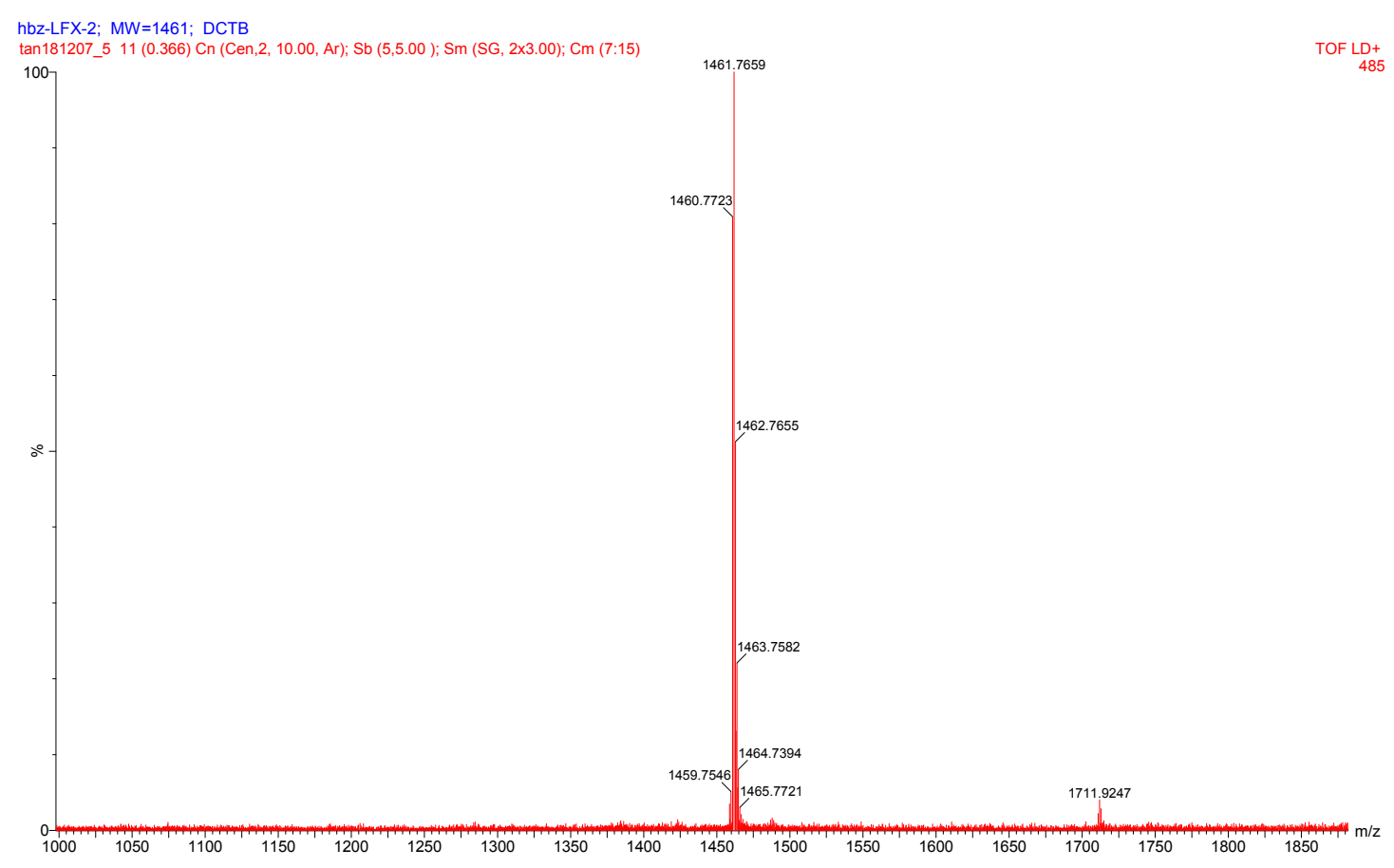

Figure S1. MALDI-TOF mass spectrum of macrocylic luminogen TPEMC.

\section{Macrocyclic luminogen TPEMCS}

A two-necked $100 \mathrm{~mL}$ round-bottom flask containing compound TPEMC (1.0eq, 140mg, $0.095 \mathrm{mmol}), \mathrm{Na}_{2} \mathrm{~S} .9 \mathrm{H}_{2} \mathrm{O}(6.0 \mathrm{eq}, 138 \mathrm{mg}, 0.57 \mathrm{mmol})$ was evacuated and refilled with argon gas for three times. Then, $20 \mathrm{~mL}$ of anhydrous THF and $20 \mathrm{~mL}$ of ethylene glycol monomethylether was added into the flask. The mixture was thenheated to $150{ }^{\circ} \mathrm{C}$ and kept stirring overnight.After evaporating the solvent, the crude product was purified via silica gel column chromatography with $\mathrm{DCM} /$ petroleum ether $=1 / 5$ as eluent to obtain $119 \mathrm{mg}$ of the title compound as white solid 
(81.2\% yield).HRMS (MALDI-TOF, DCTB as matrix) m/z: [M]+ calcd. $\operatorname{forC}_{108} \mathrm{H}_{104} \mathrm{O}_{4} \mathrm{~S}_{2}$ : 1529.7410. Found: 1529.7452. ${ }^{1} \mathrm{H}$ NMR (600 MHz, $\left.\mathrm{CDCl}_{3}\right) \delta / \mathrm{ppm}: 7.79(\mathrm{~s}, 4 \mathrm{H}), 7.61$ (d, 4H, $J=$ $7.7 \mathrm{~Hz}), 7.48$ (d, 4H, $J=7.8 \mathrm{~Hz}), 7.42$ (t, 4H, $J=7.7 \mathrm{~Hz}), 7.39$ (m, 8H, $J=8.4 \mathrm{~Hz}), 7.35$ (s, 4H), $7.14(\mathrm{~d}, 8 \mathrm{H}, J=8.4 \mathrm{~Hz}), 7.01(\mathrm{~d}, 8 \mathrm{H}, J=8.8 \mathrm{~Hz}), 6.66(\mathrm{~m}, 8 \mathrm{H}, J=8.8 \mathrm{~Hz}), 3.87(\mathrm{t}, 8 \mathrm{H}, J=6.7$ $\mathrm{Hz}), 1.73(\mathrm{q}, 8 \mathrm{H}, J=7.2 \mathrm{~Hz}), 1.41(\mathrm{~m}, 8 \mathrm{H}), 1.32-1.29(\mathrm{~m}, 16 \mathrm{H}), 0.88(\mathrm{t}, 12 \mathrm{H}, J=7.2 \mathrm{~Hz}) .{ }^{13} \mathrm{C}$ NMR (151 MHz, $\left.\mathrm{CDCl}_{3}\right) \delta 156.76,142.82,142.59,140.83,137.18,135.01,133.58,131.62$, $130.85,128.16,125.32,124.88,124.15,122.99,122.50,112.58,66.80,30.91,30.62,28.68,28.28$, $24.75,21.68,13.11$.

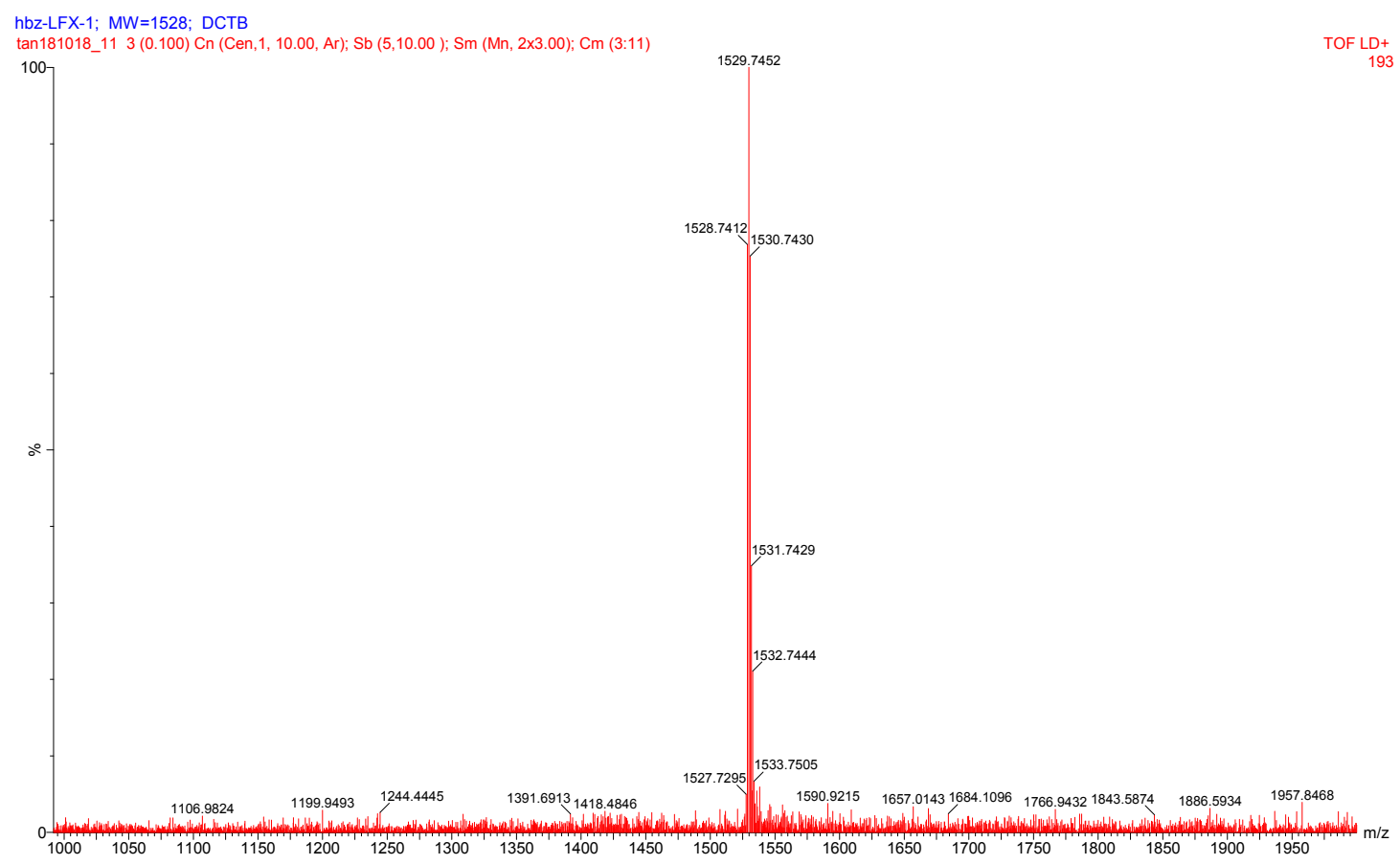

Figure S2. MALDI-TOF mass spectrum of macrocylic luminogen TPEMCS. 


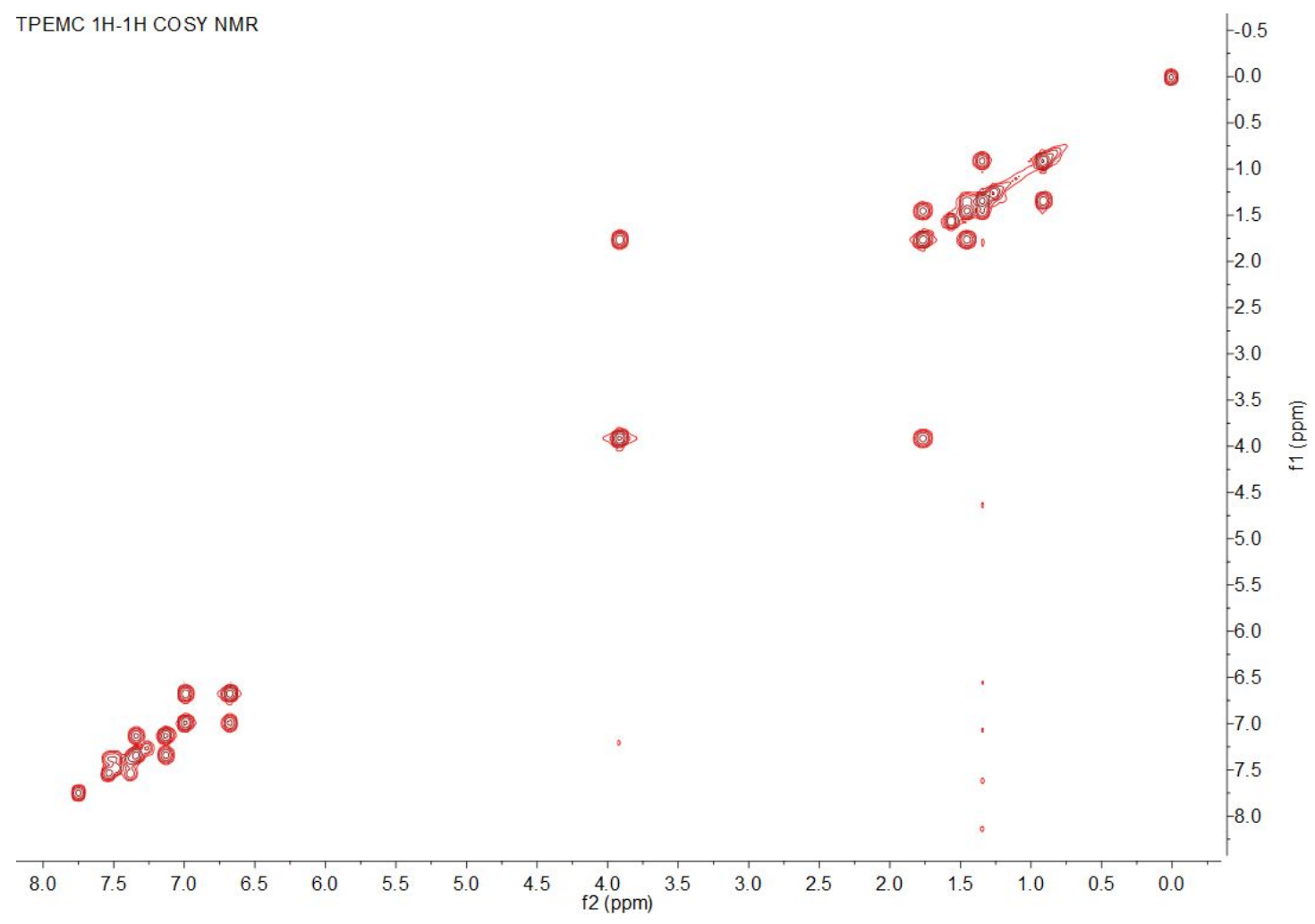

Figure S3. $2 \mathrm{D}{ }^{1} \mathrm{H}-{ }^{-1} \mathrm{H}$ COSY spectrum of TPEMC in $\mathrm{CDCl}_{3}$ at $298 \mathrm{~K}$.

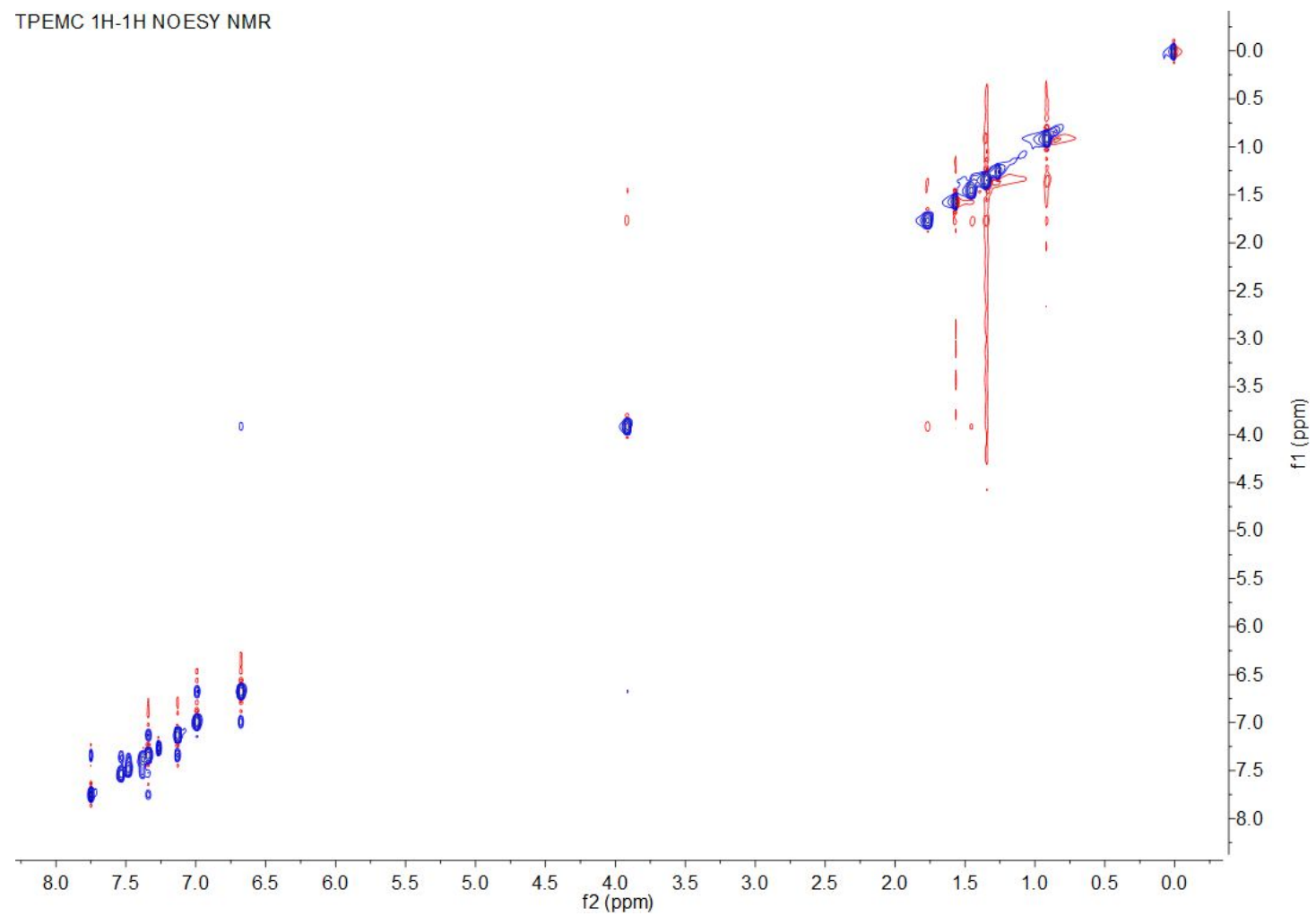

Figure S4. 2D ${ }^{1} \mathrm{H}-{ }^{1} \mathrm{H}$ NOESY spectrum of TPEMC in $\mathrm{CDCl}_{3}$ at $298 \mathrm{~K}$. 


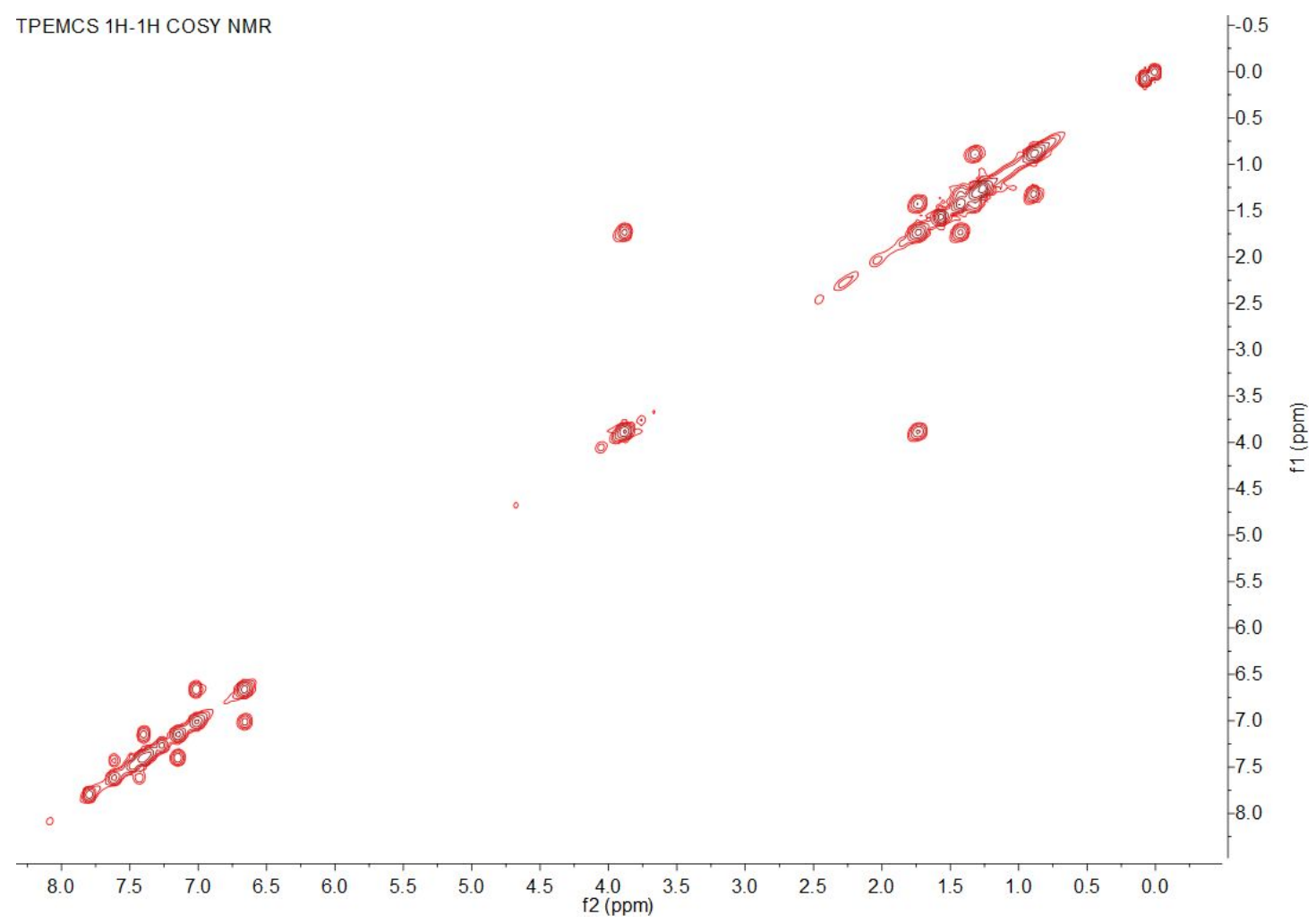

Figure S5. 2D ${ }^{1} \mathrm{H}-{ }^{1} \mathrm{H}$ COSY spectrum of TPEMCS in $\mathrm{CDCl}_{3}$ at $298 \mathrm{~K}$.

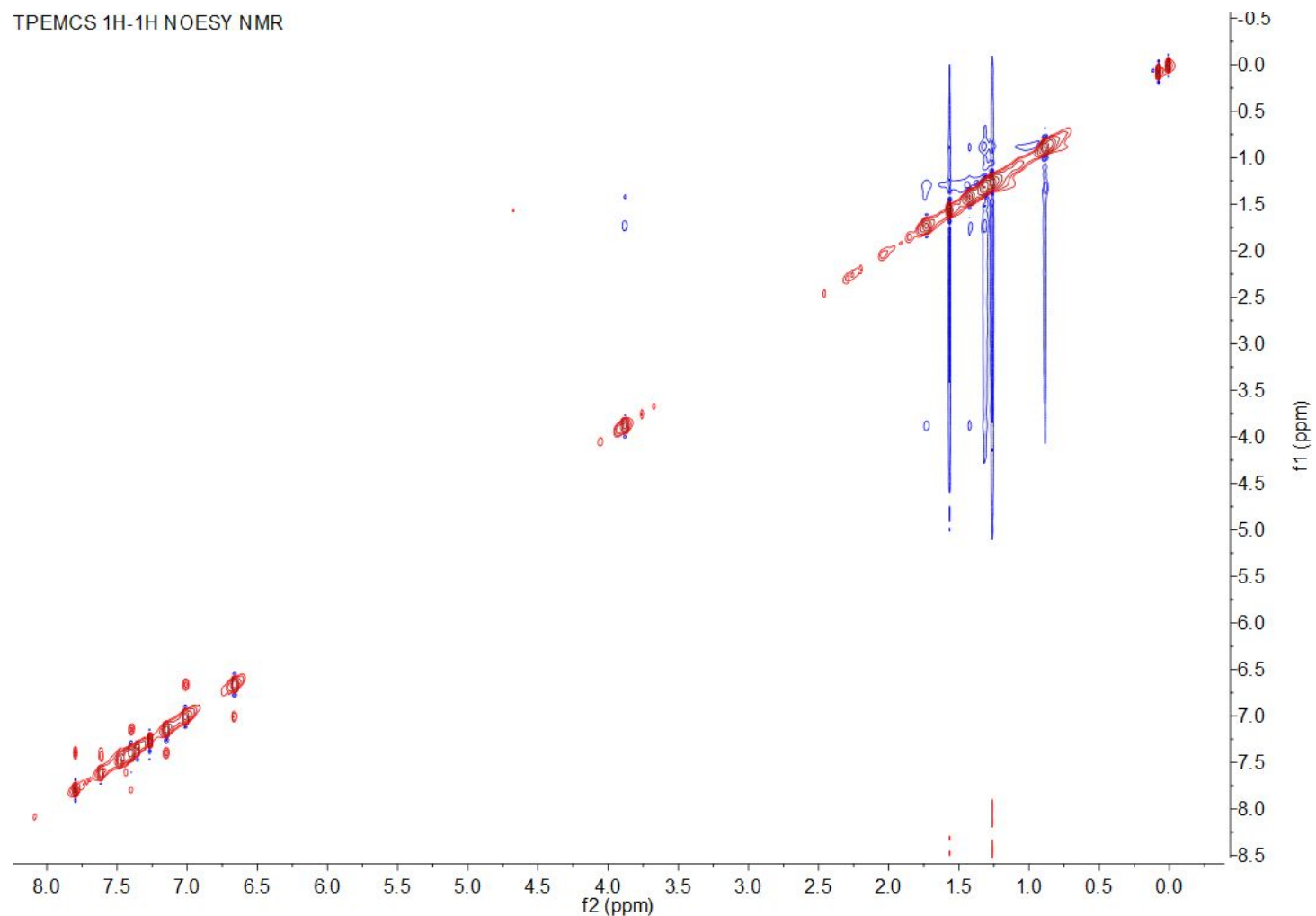

Figure S6. $2 \mathrm{D}{ }^{1} \mathrm{H}-{ }^{1} \mathrm{H}$ NOESY spectrum of TPEMCS in $\mathrm{CDCl}_{3}$ at $298 \mathrm{~K}$. 


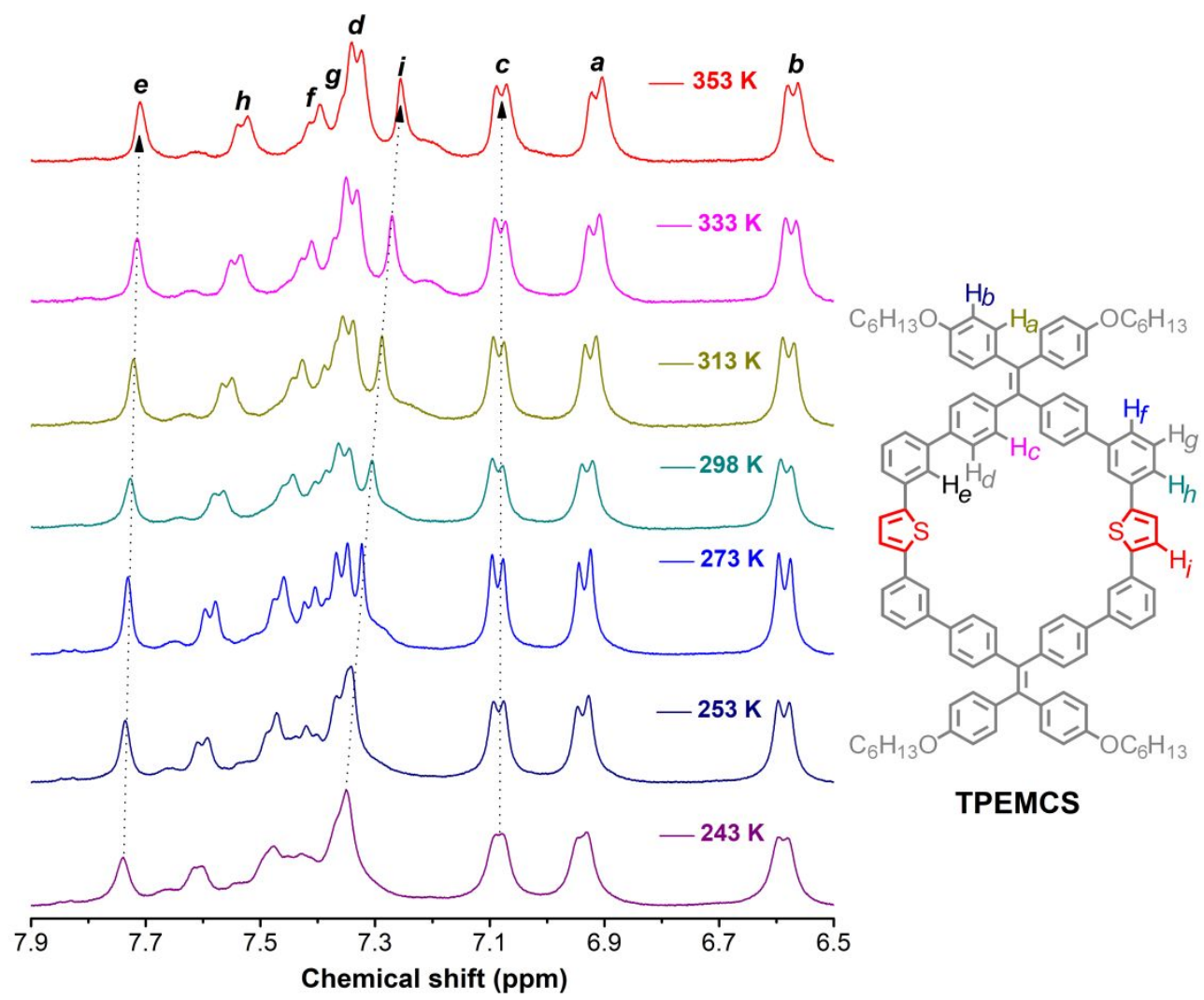

Figure S7. Aromatic region of variable temperature ${ }^{1} \mathrm{H}$ NMR spectrum of TPEMCS in $\mathrm{C}_{2} \mathrm{D}_{2} \mathrm{Cl}_{4}$.

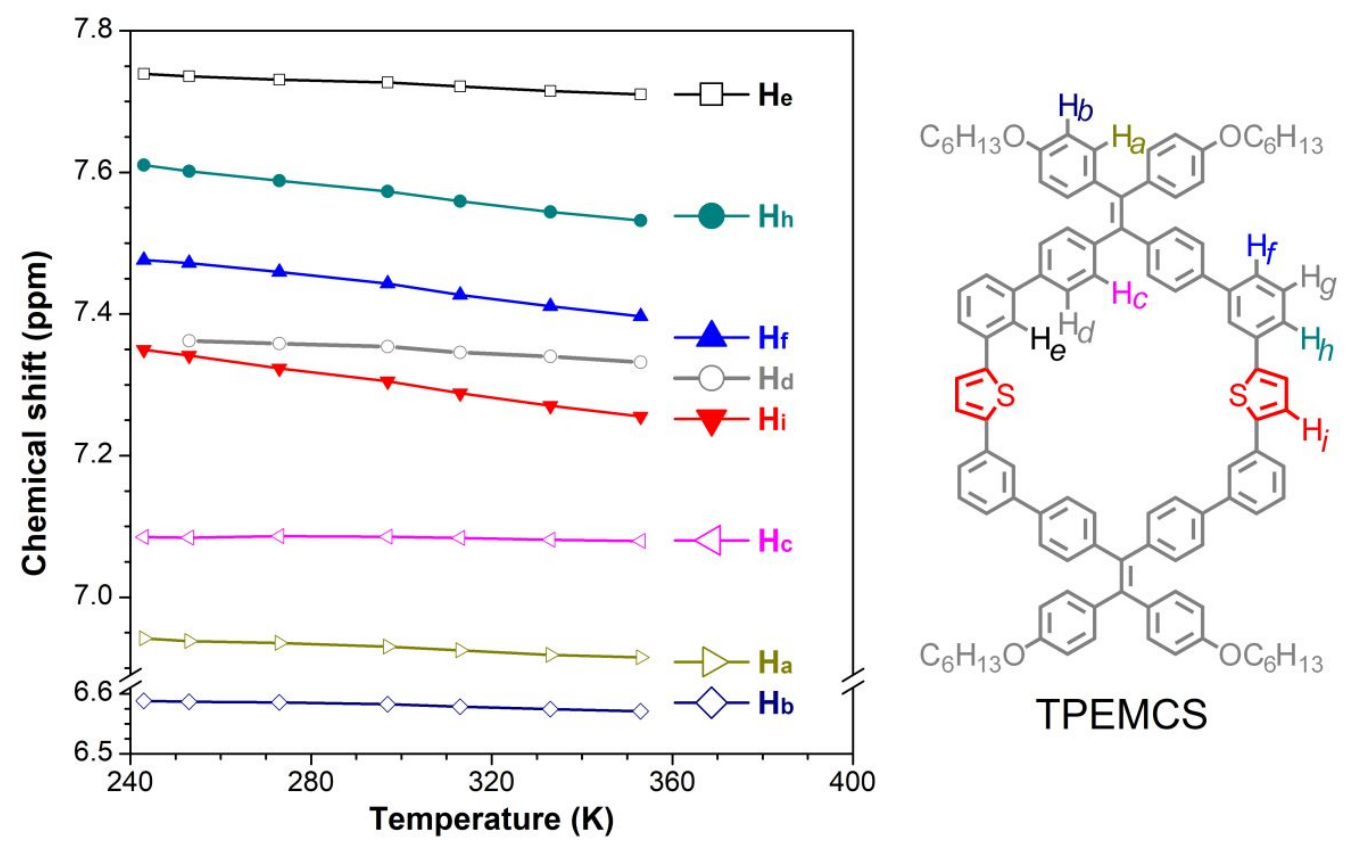

Figure S8. Curves of the chemical shifts for different aromatic protons on TPEMCS against NMR measurement temperature. 


\section{Figures and charts}

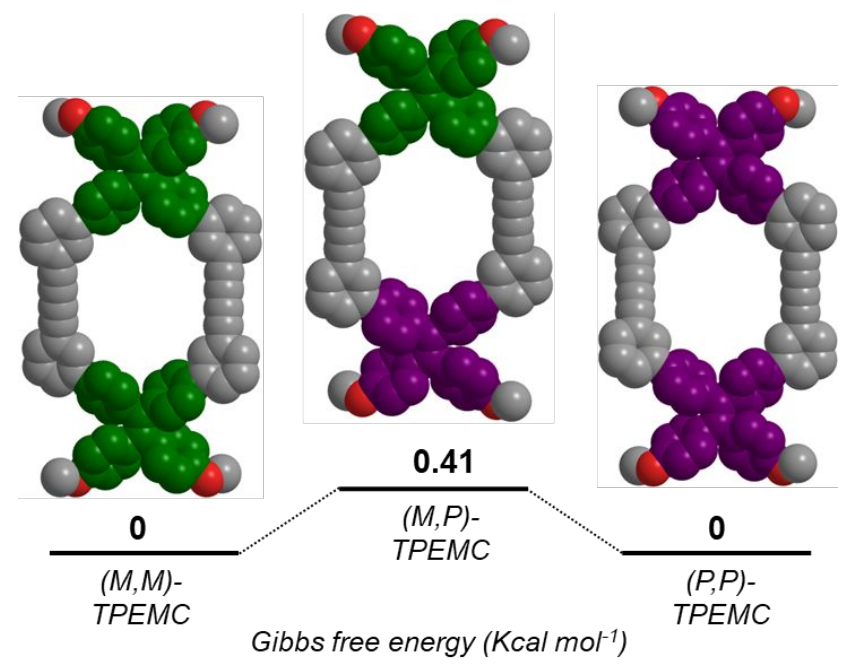

3.

Figure S9. Molecular models and energy of conformational isomers for TPEMC as calculated at the B3LYP/6-31G(d) level of DFT.
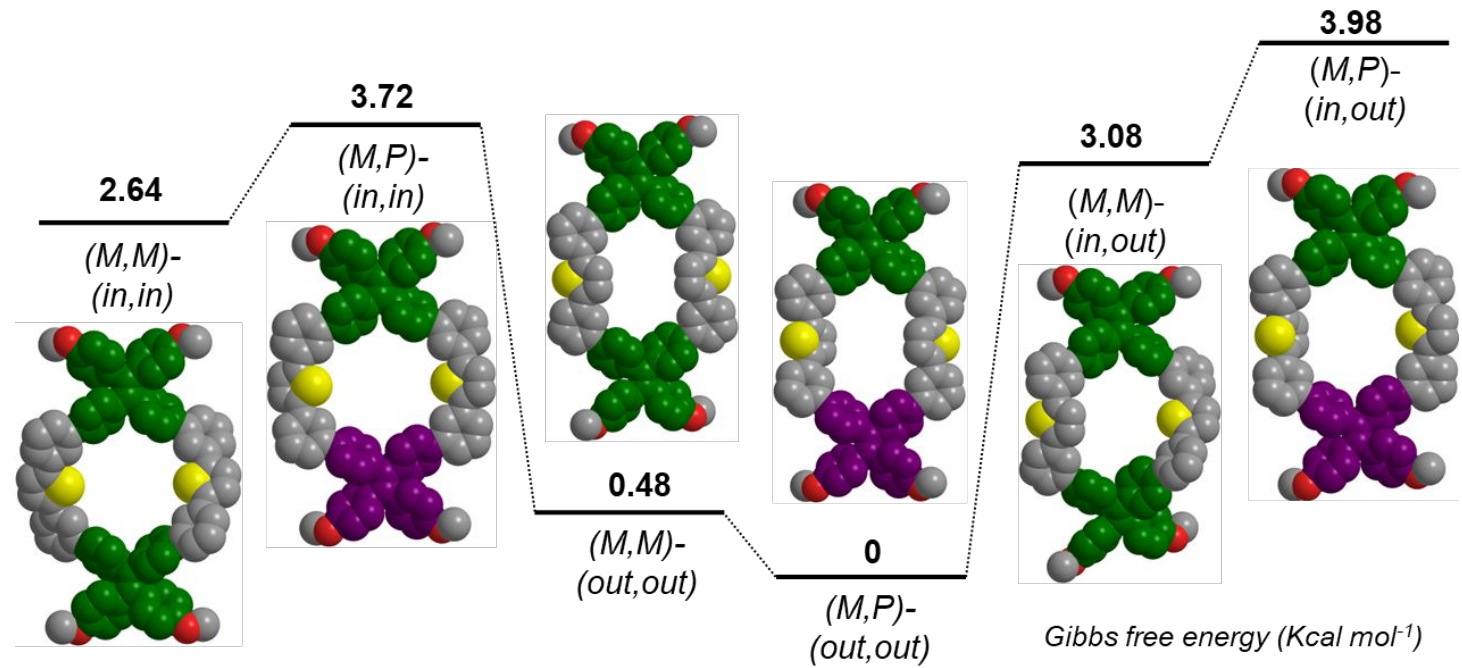

Figure S10. Molecular models and energy of conformational isomers for TPEMCS as calculated at the B3LYP/6-31G(d) level of DFT. "out" means the S atom is located outside the macrocycle framework, whereas "in" means the $\mathrm{S}$ atom is located inside the macrocycle. (Hydrogen atoms are removed for clarity.) 

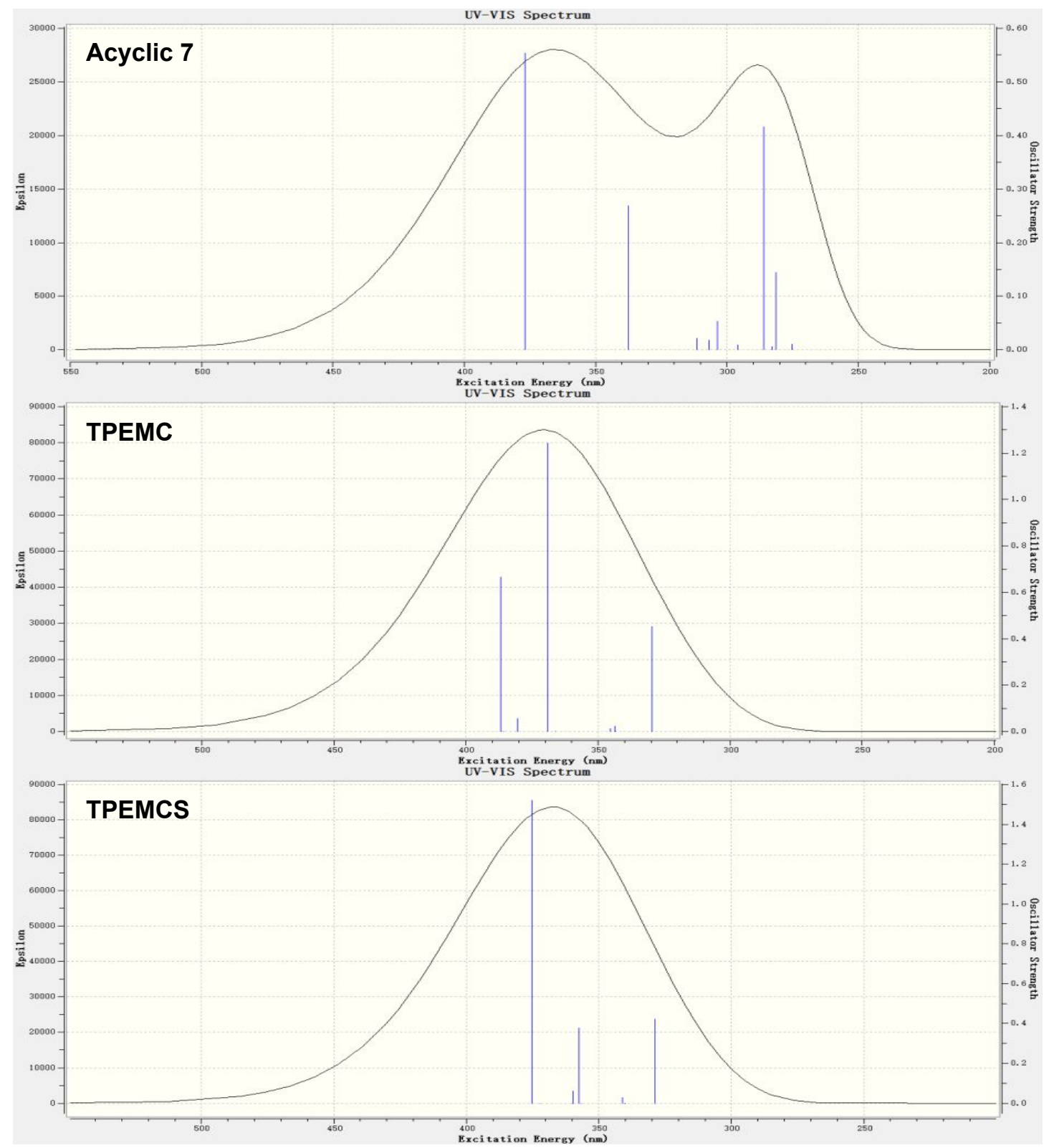

Figure S11. UV-Vis absorption spectra of acyclic precursor 7 (top), TPEMC (middle), and TPEMCS (bottom) calculated based on TDDFT with $\mathrm{n}=10$ roots using functional B3LYP with the 6-31G basis set. 

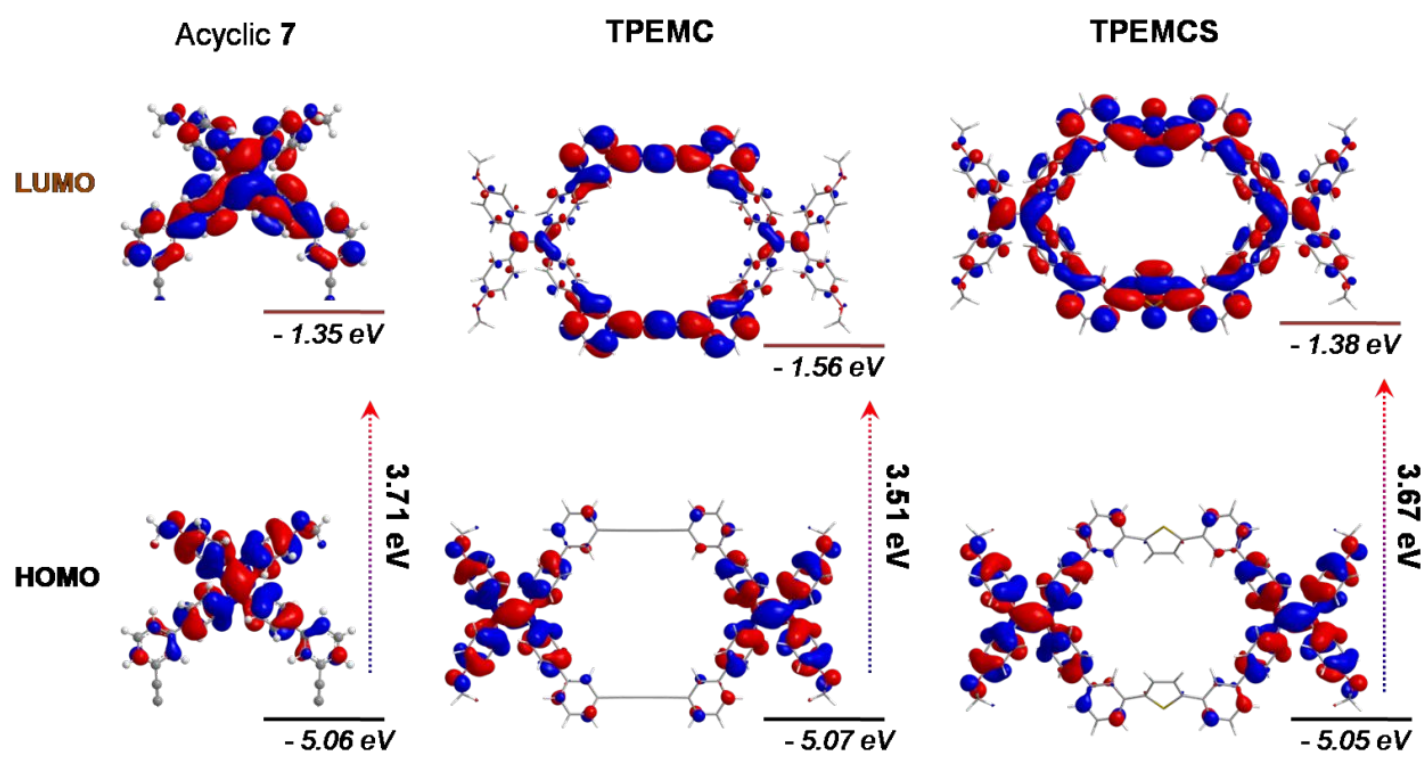

Figure S12. Molecular energy level of model compounds for acyclic precursor 7, TPEMC, and TPEMCS respectively, based on B3LYP/6-31G level via DFT calculation (hexyl chain was substituted with a methyl group to reduce the calculation time).

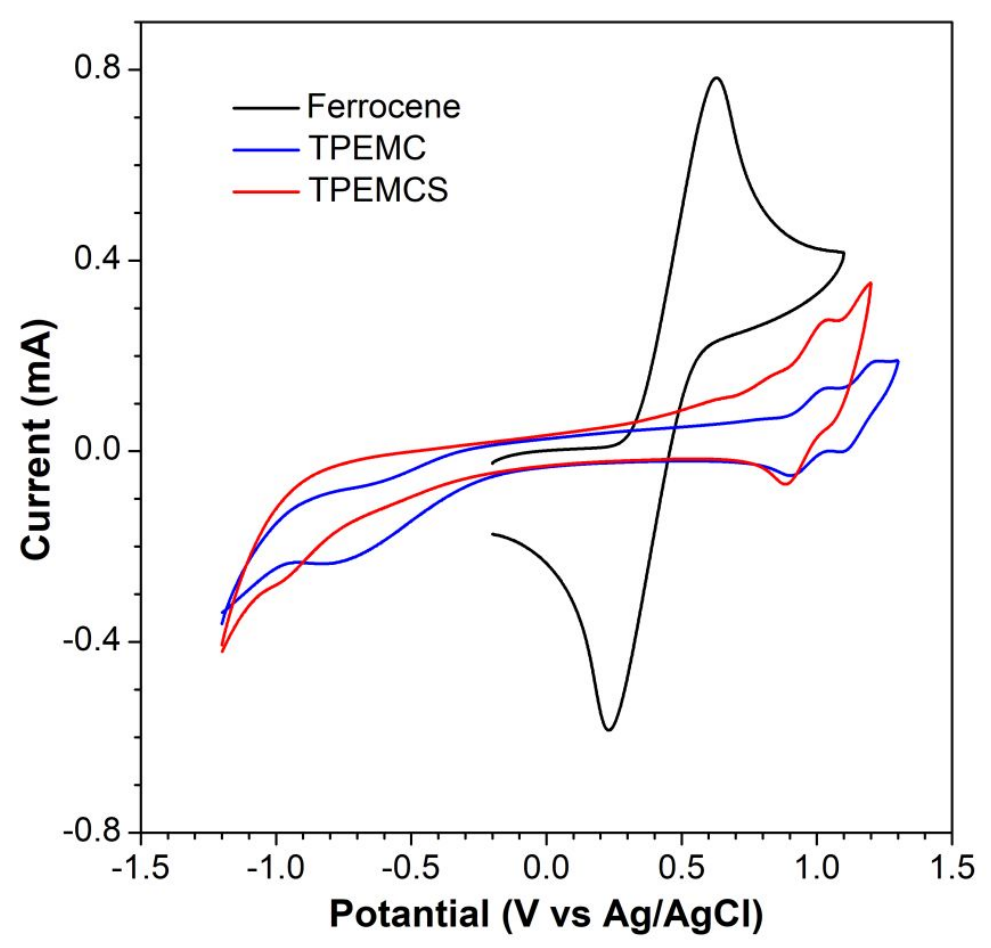

Figure S13. Cyclic voltametry spectra of ferrocene, TPEMC, and TPEMCS in DCM $(1 \mathrm{mM})$ measured with $[\mathrm{n}-\mathrm{Bu} 4 \mathrm{~N}][\mathrm{PF} 6](0.1 \mathrm{M})$ as a supporting electrolyte. 

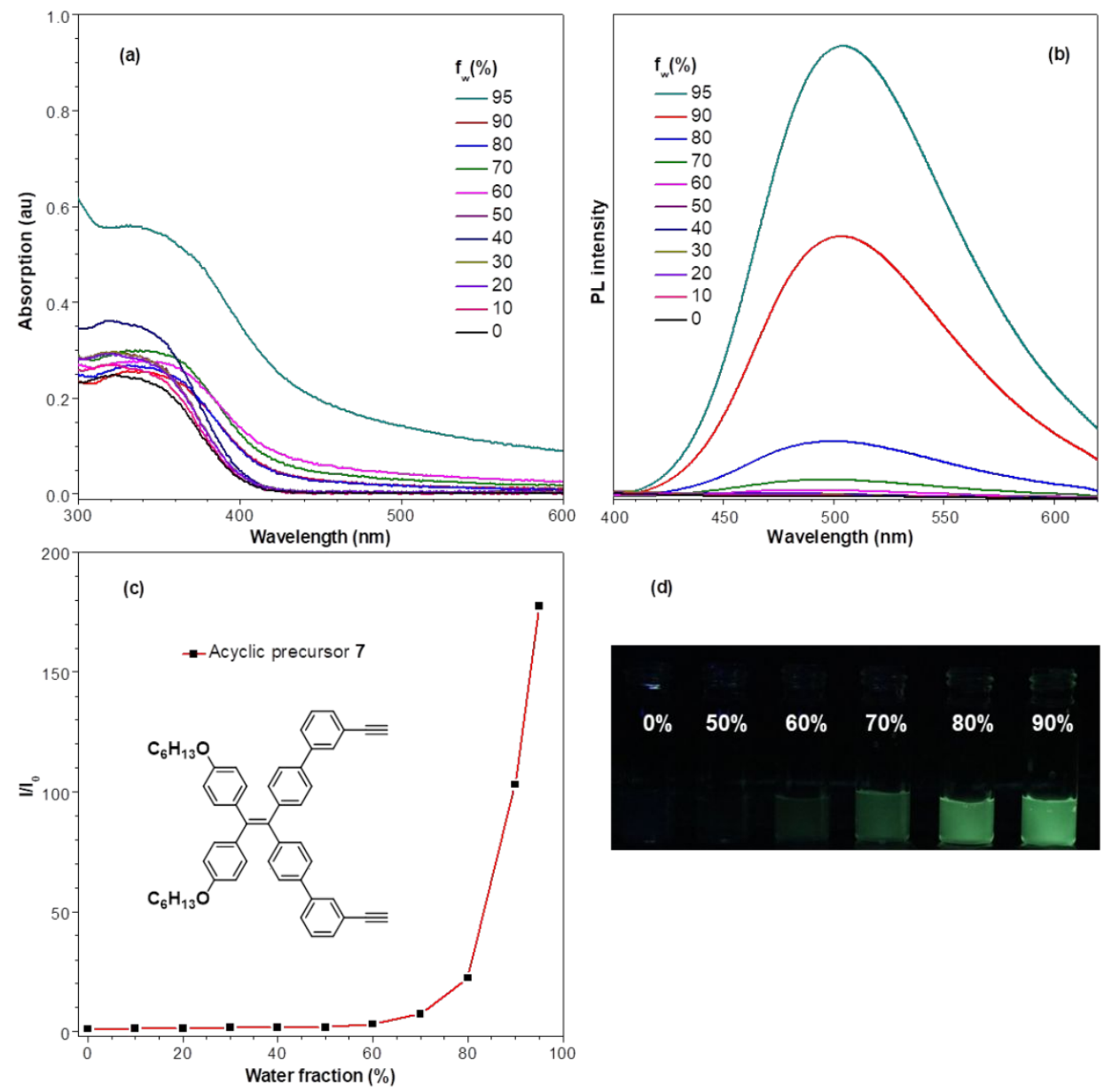

(d)

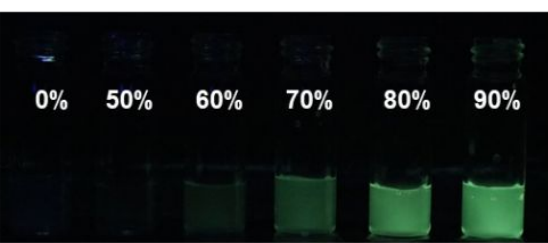

Figure S14. (a) UV-Vis absorption spectra and (b) photoluminescence spectra of acyclic precursor 7 in THF and THF/water mixtures with different water fraction $\left(f_{w}\right)$. (c) Plots of the maximum emission intensities of 7 in THF and THF/water mixtures versus $f_{w}$. (d) Images of 7 under UV lamp in THF and THF/water mixture with different $\mathrm{f}_{\mathrm{w}}$. Concentration $=10 \mu \mathrm{M}$. 

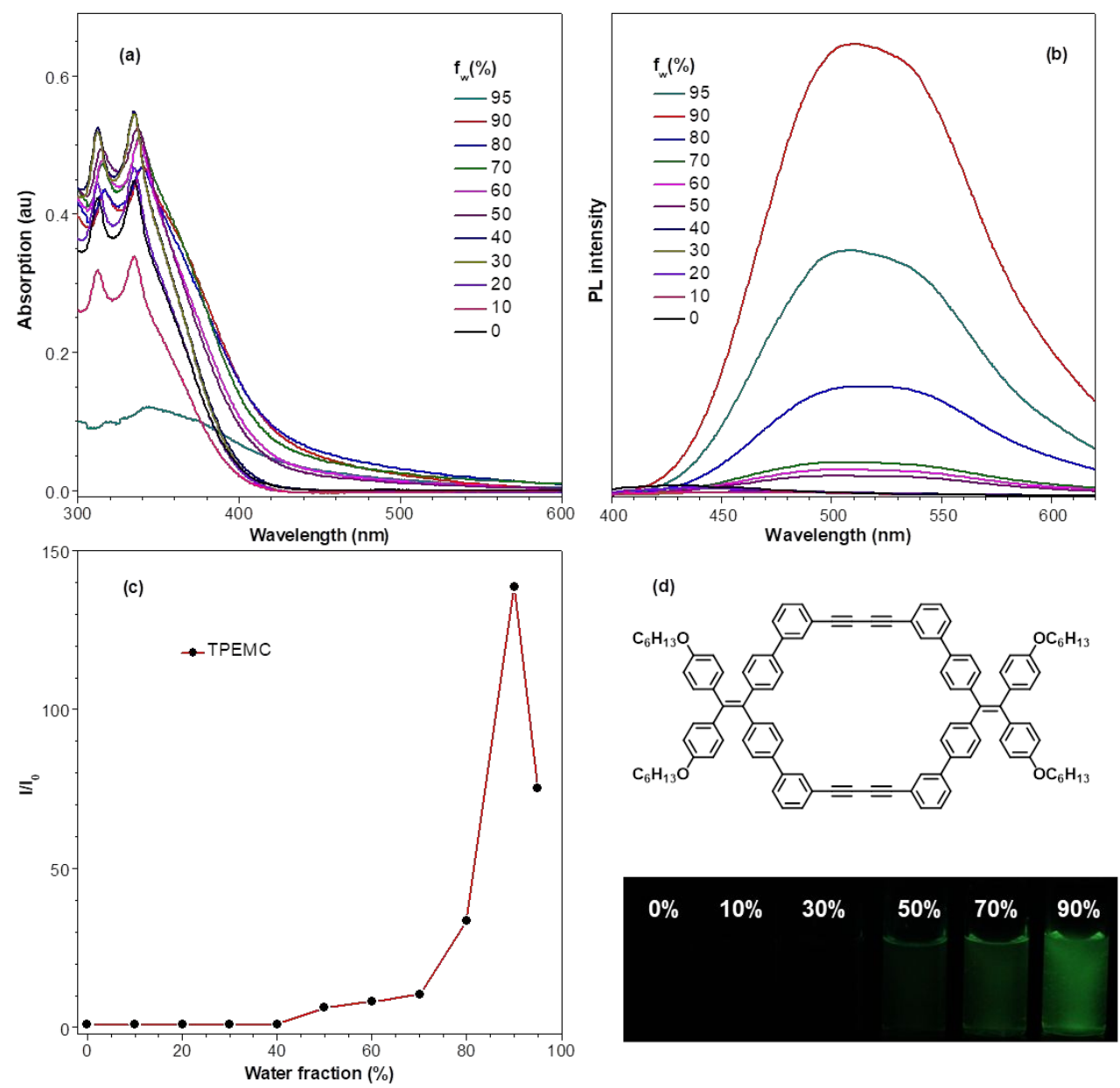

Figure S15. (a) UV-Vis absorption spectra and (b) photoluminescence spectra of TPEMC in THF and THF/water mixtures with different water fraction $\left(f_{w}\right)$. (c) Plots of the maximum emission intensities of TPEMC in THF and THF/water mixtures versus $f_{w}$. (d) Images of TPEMC under UV lamp in THF and THF/water mixtures with different $\mathrm{f}_{\mathrm{w}}$. Concentration $=10 \mu \mathrm{M}$. 

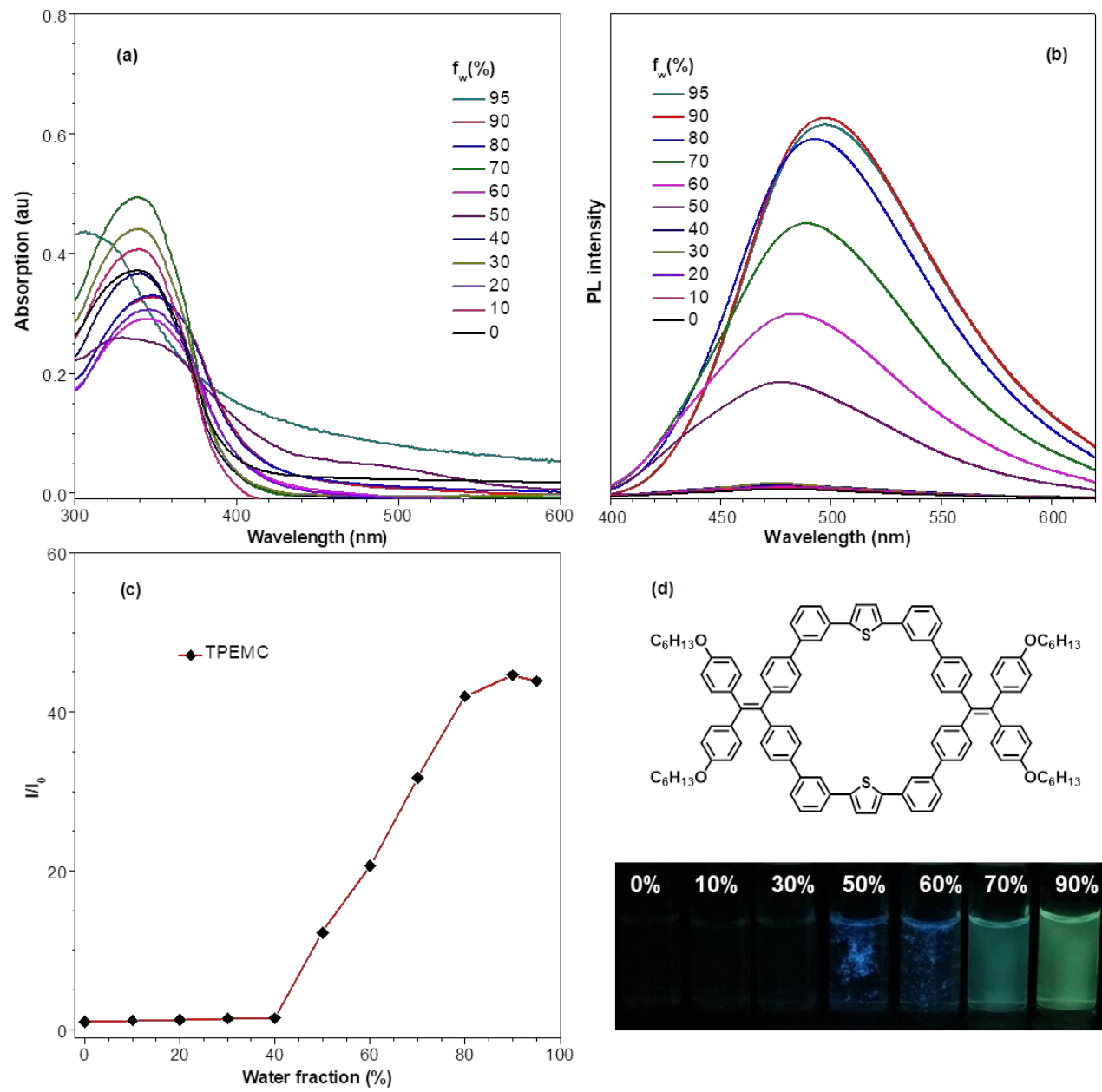

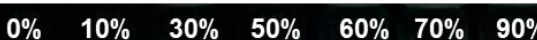

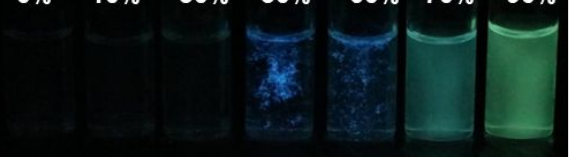

Figure S16. (a) UV-Vis absorption spectra and (b) photoluminescence spectra of TPEMCS in THF and THF/water mixtures with different water fraction $\left(f_{w}\right)$. (c) Plots of the maximum emission intensities of TPEMCS in THF and THF/water mixtures versus $f_{w}$. (d) Images of TPEMCS under UV lamp in THF and THF/water mixtures with different $\mathrm{f}_{\mathrm{w}}$. Concentration $=10$ $\mu \mathrm{M}$. 


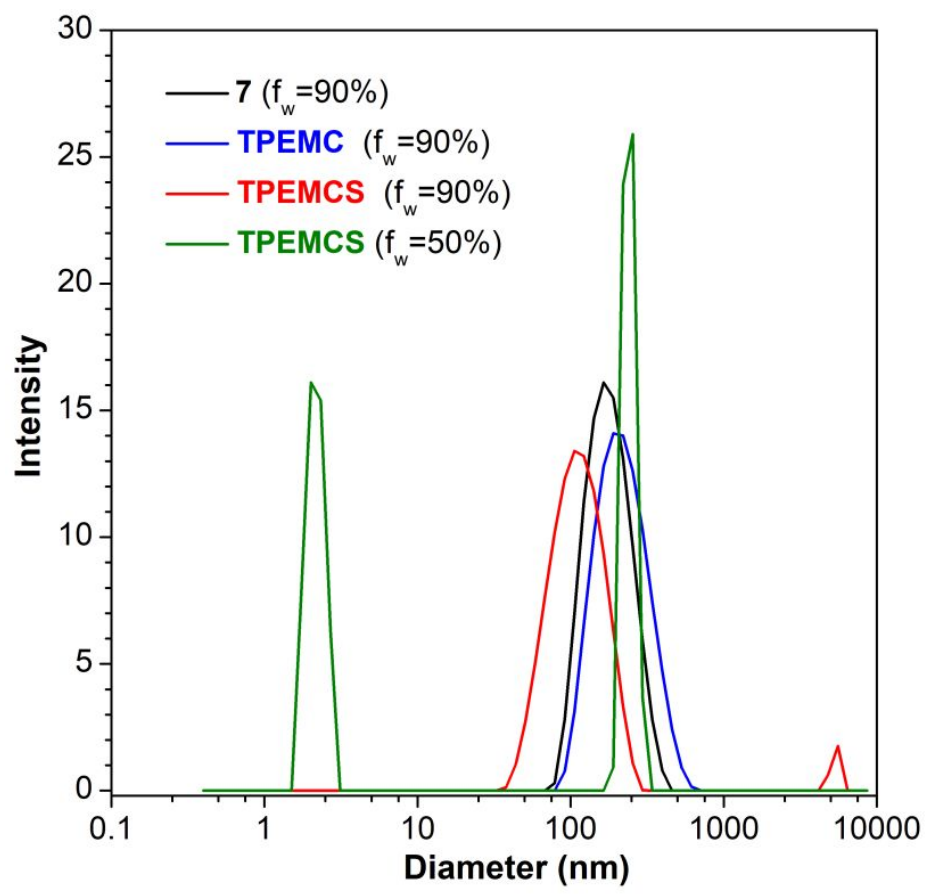

Figure S17. Dynamic light scattering of acyclic precursor 7, TPEMC, and TPEMCS in $\mathrm{THF} /$ Water mixture with water fraction $f_{w}=50 \%$ or $90 \%$.
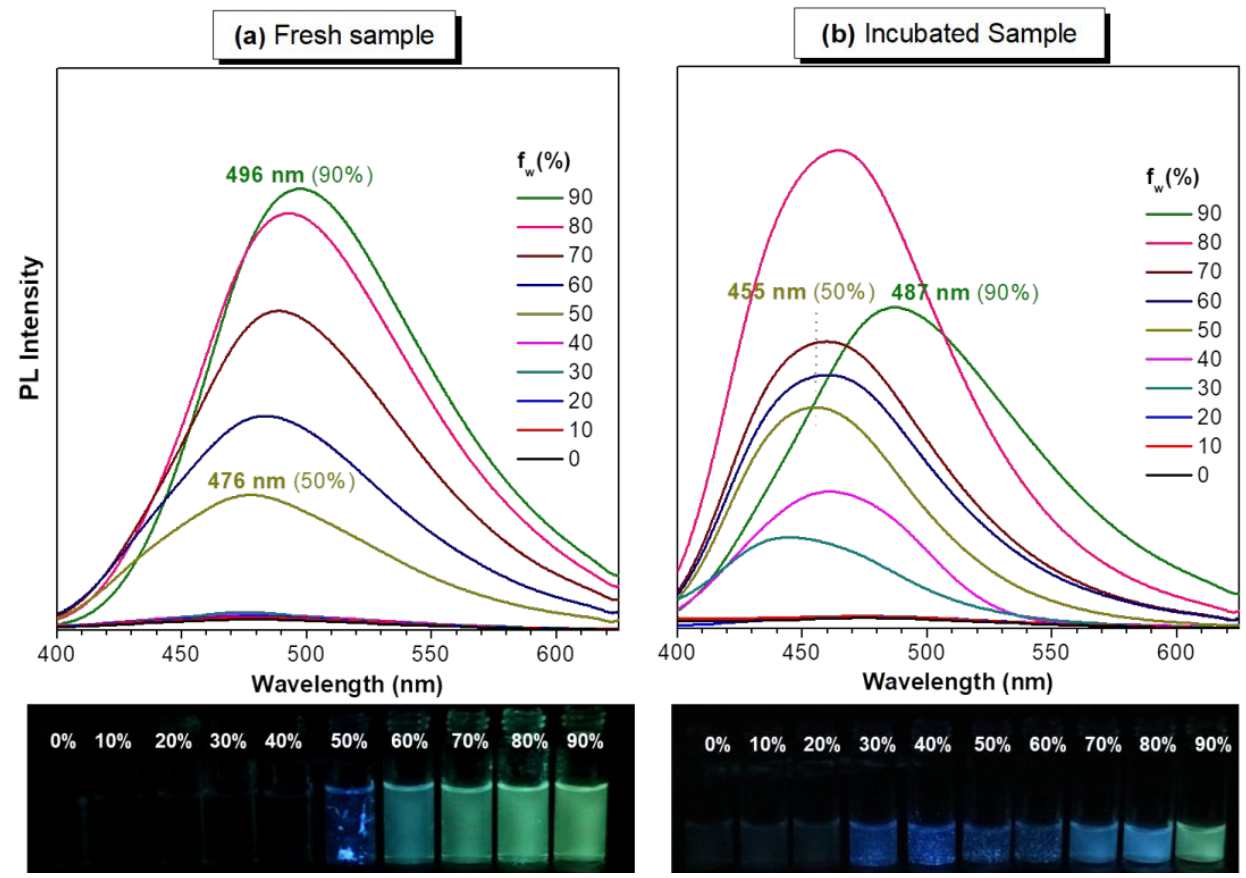

Figure S18. Fluorescent spectra and images of (a) fresh-prepared and (b) incubated (for 7 days) samples of TPEMCS in THF/water mixture with different water fraction. 

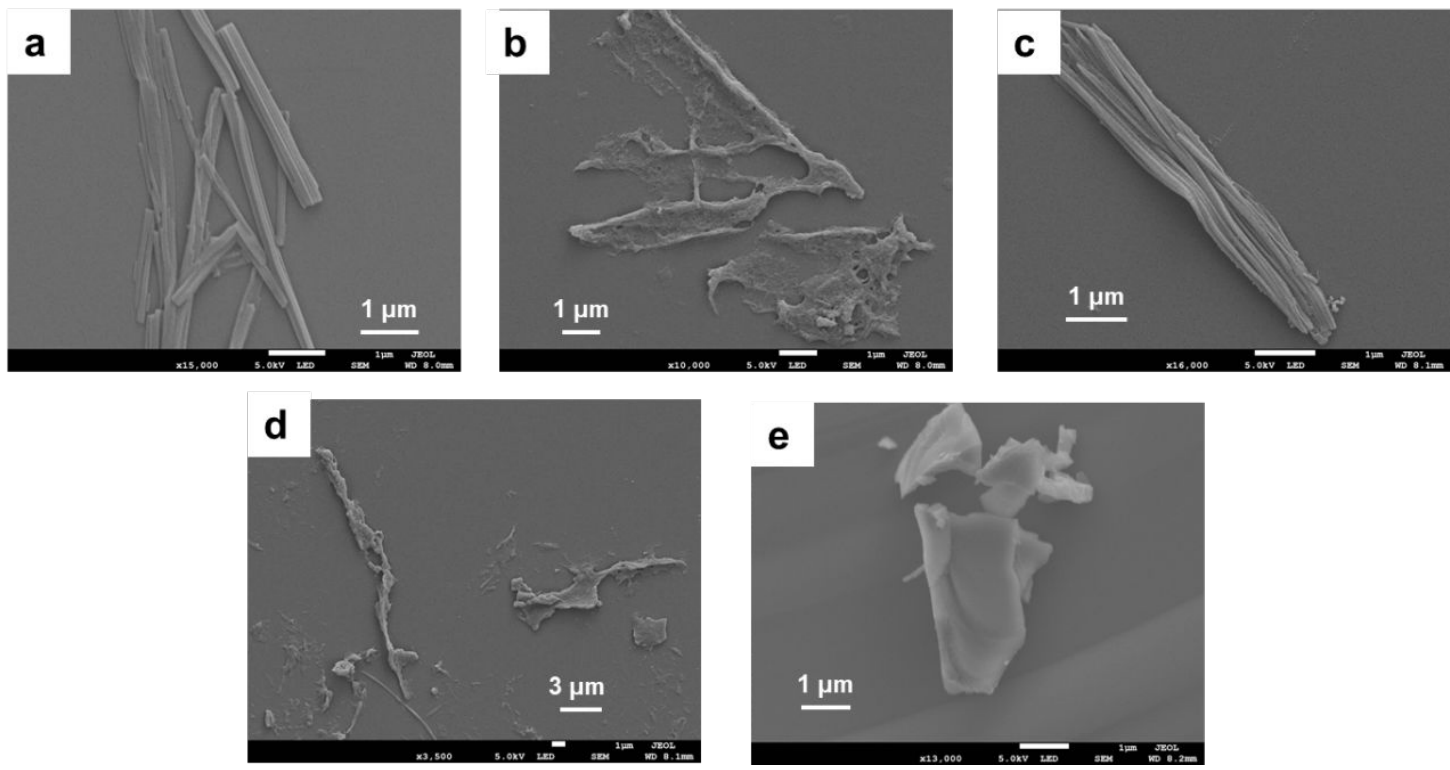

Figure S19. SEM images of the self-organized microstructures of TPEMCS formed in the THF/water mixtures at (a) $f_{w}=30 \%$, (b) $f_{w}=40 \%$, (c) $f_{w}=50 \%$, (d) $f_{w}=60 \%$, and (e) $f_{w}=90 \%$ after standing under ambient conditions for 7 days (Concentration: $10 \mu \mathrm{M}$ ).
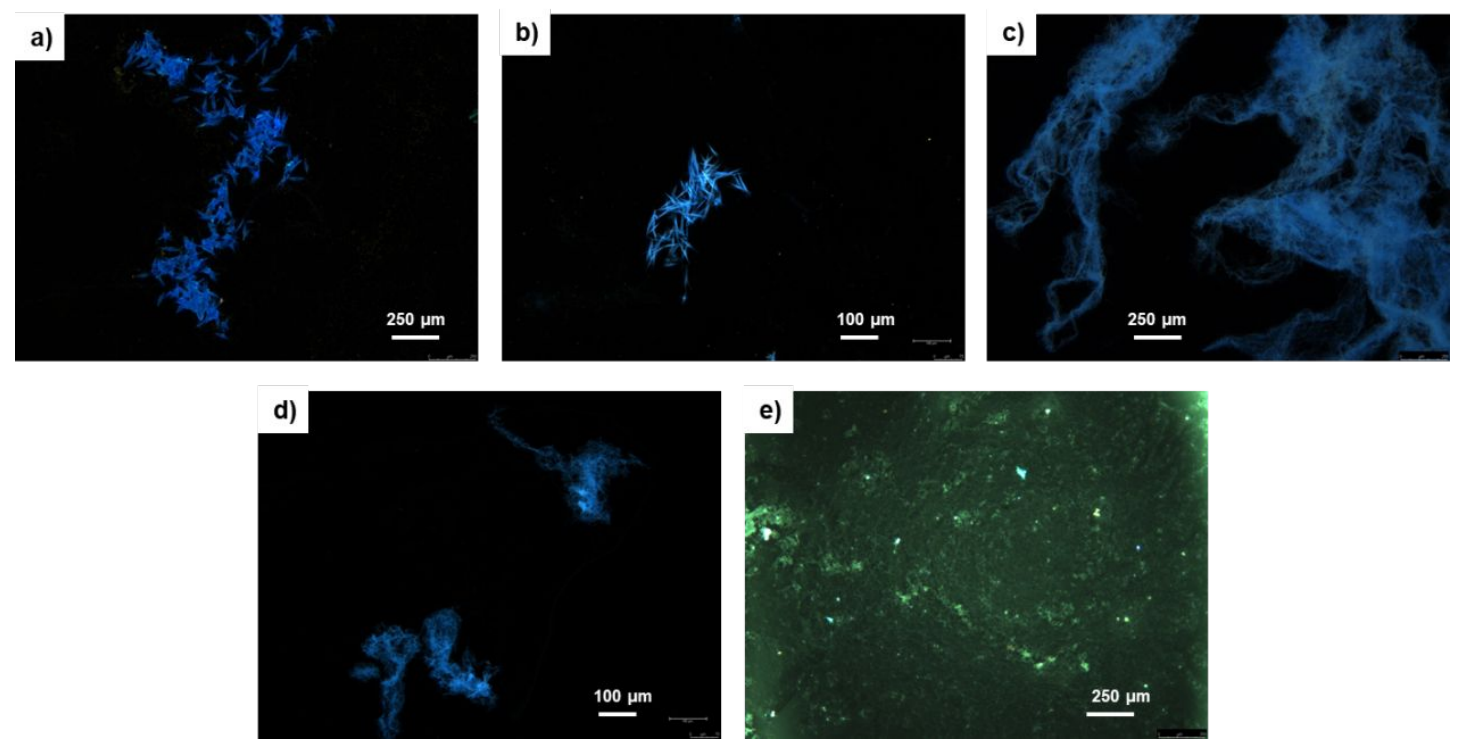

Figure S20. Photoluminescent images of the self-organized microstructures of TPEMCS formed in the THF/water mixtures at (a) $f_{w}=30 \%$, (b) $f_{w}=40 \%$, (c) $f_{w}=50 \%$, (d) $f_{w}=60 \%$, and (e) $f_{w}=$ 90\% after standing under ambient conditions for 7 days (Concentration: $10 \mu \mathrm{M}$ ). 

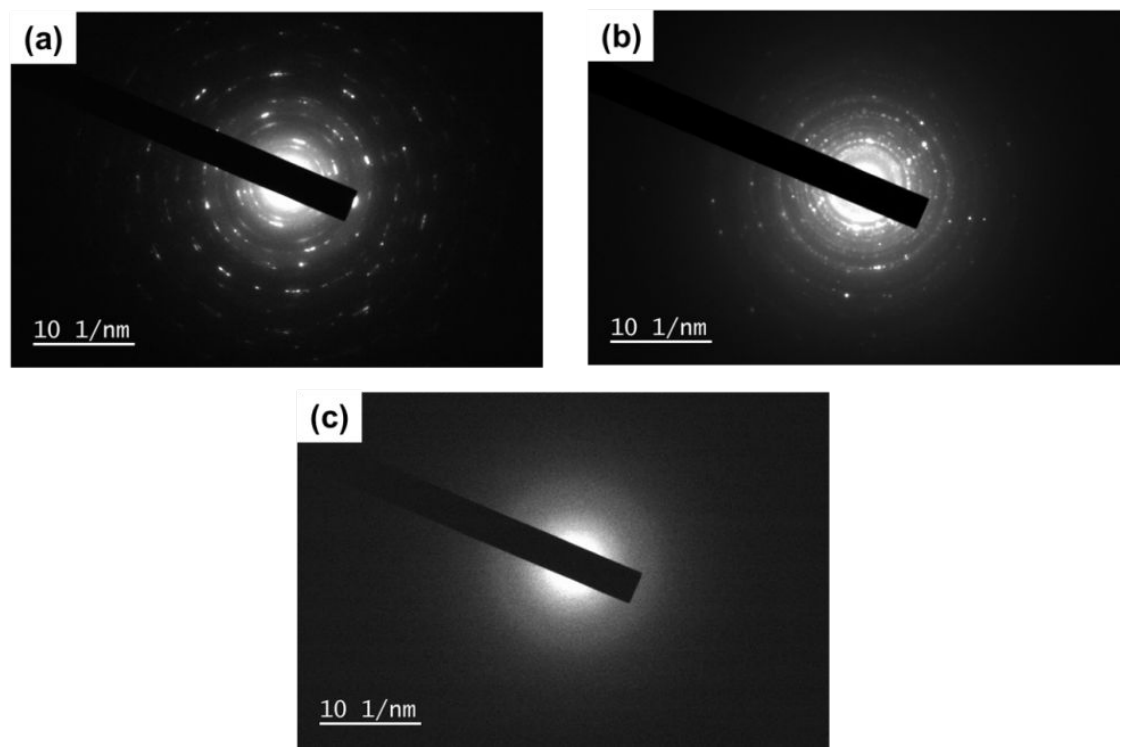

Figure S21. TEM electron diffraction of the self-organized microstructures of TPEMCS formed in the THF/water mixtures at (a) $f_{w}=30 \%$, (b) $f_{w}=50 \%$, and (c) $f_{w}=90 \%$ after standing under ambient conditions for 7 days (Concentration: $10 \mu \mathrm{M}$ ). The same pattern was confirmed at several different spots.
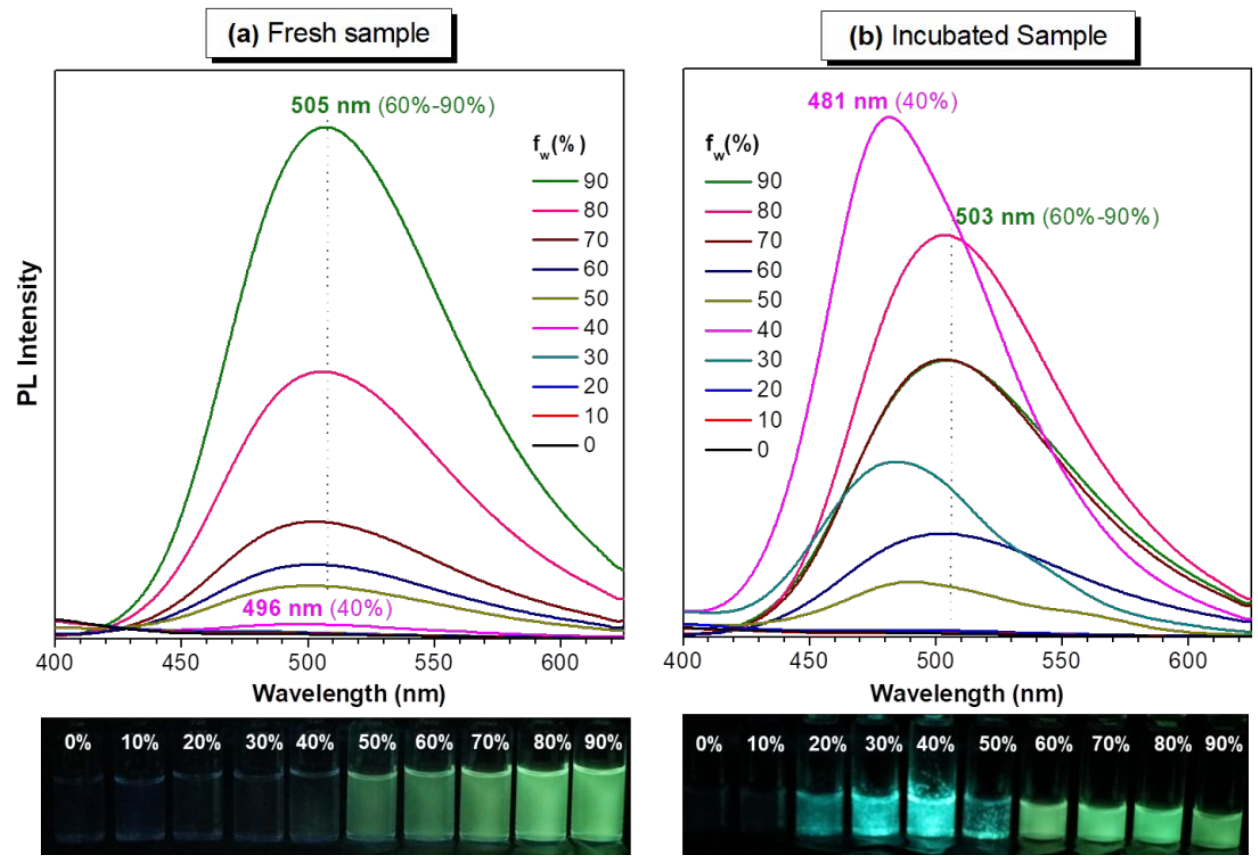

Figure S22. Fluorescent spectra and images of (a) fresh-prepared and (b) incubated (for 7 days) samples of TPEMC in THF/water mixture with different water fraction. 

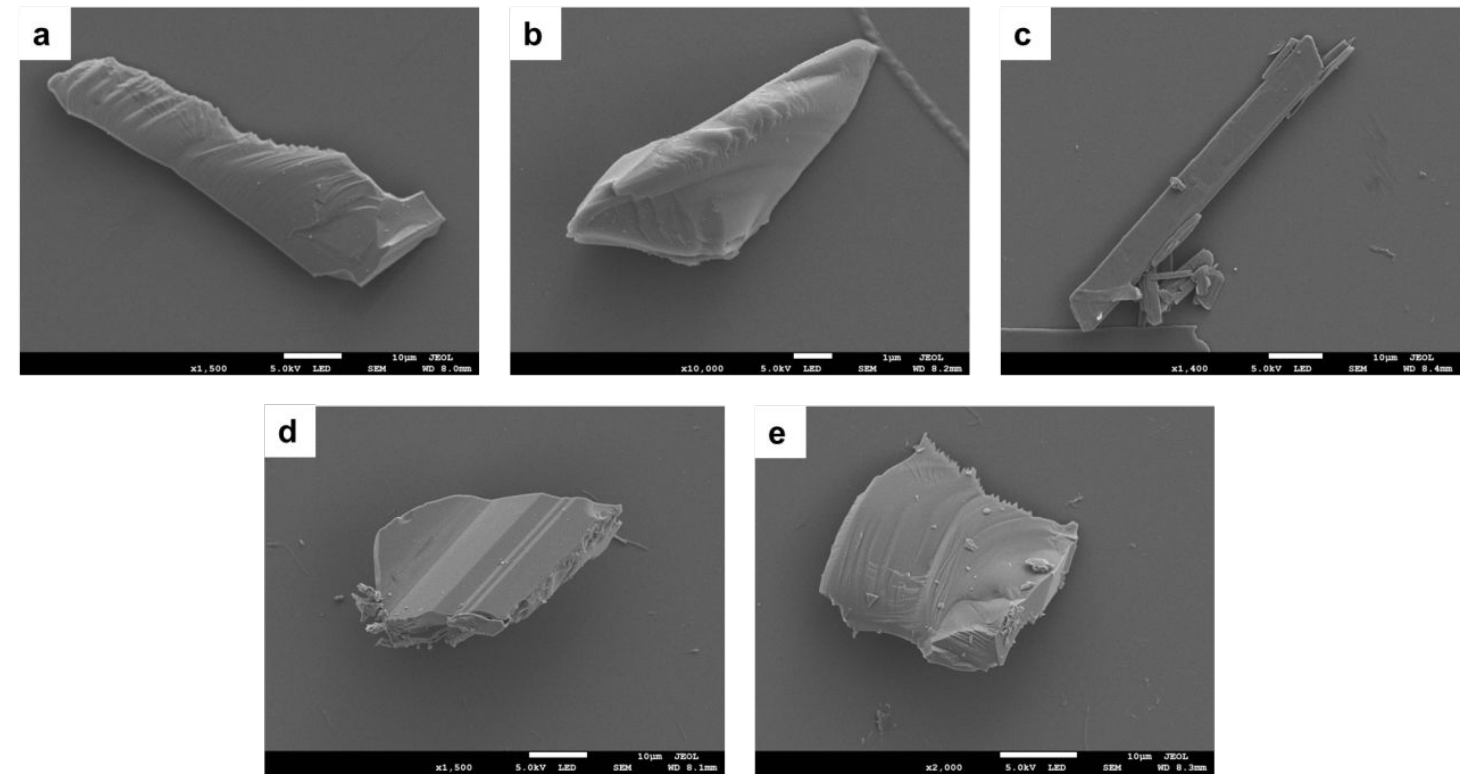

Figure S23. SEM images of the self-organized microstructures of TPEMC formed in the THF/water mixtures at (a) $f_{w}=30 \%$, (b) $f_{w}=40 \%$, (c) $f_{w}=50 \%$, (d) $f_{w}=60 \%$, and (e) $f_{w}=90 \%$ after standing under ambient conditions for 7 days (Concentration: $10 \mu \mathrm{M}$ ).
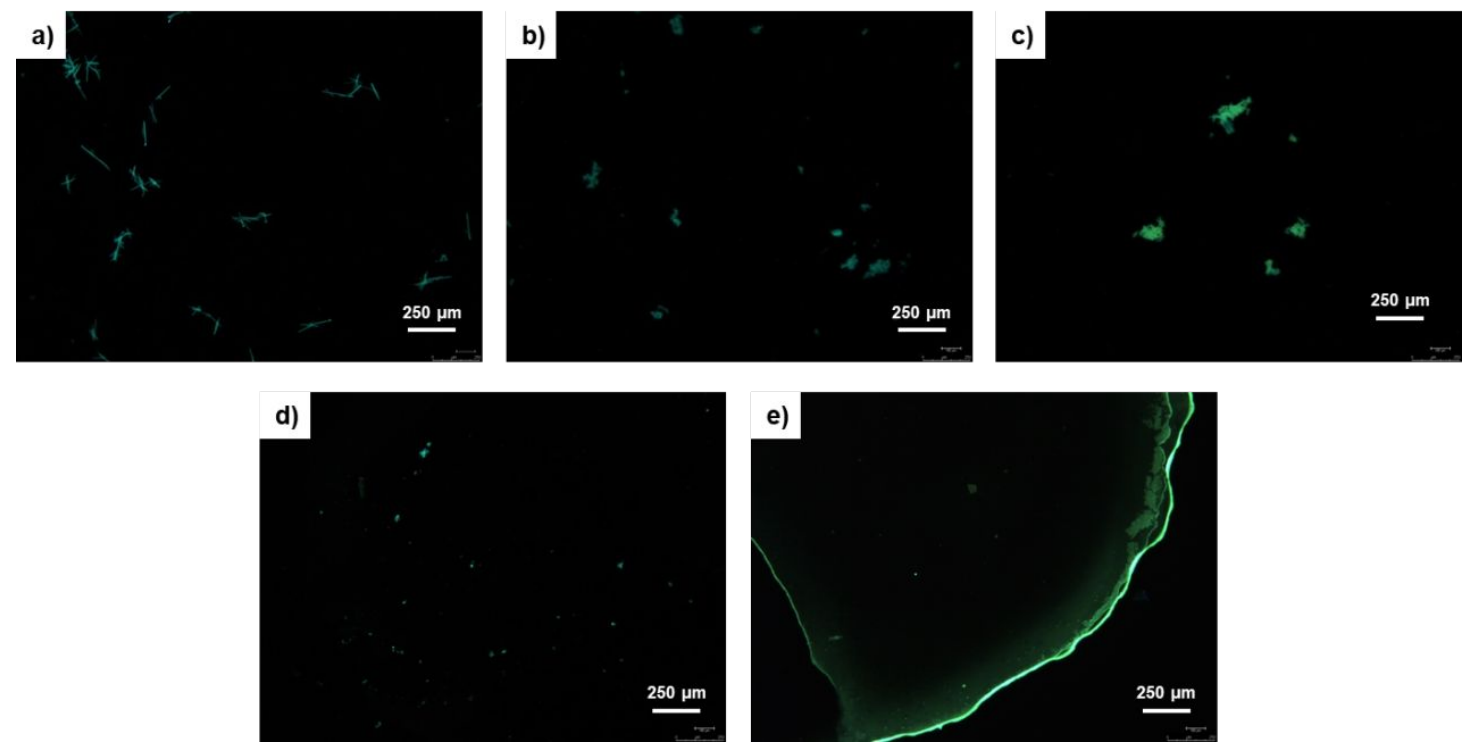

Figure S24. Photoluminescent images of the self-organized microstructures of TPEMC formed in the THF/water mixtures at (a) $f_{w}=30 \%$, (b) $f_{w}=40 \%$, (c) $f_{w}=50 \%$, (d) $f_{w}=60 \%$, and (e) $f_{w}=90 \%$ after standing under ambient conditions for 7 days (Concentration: $10 \mu \mathrm{M})$. 


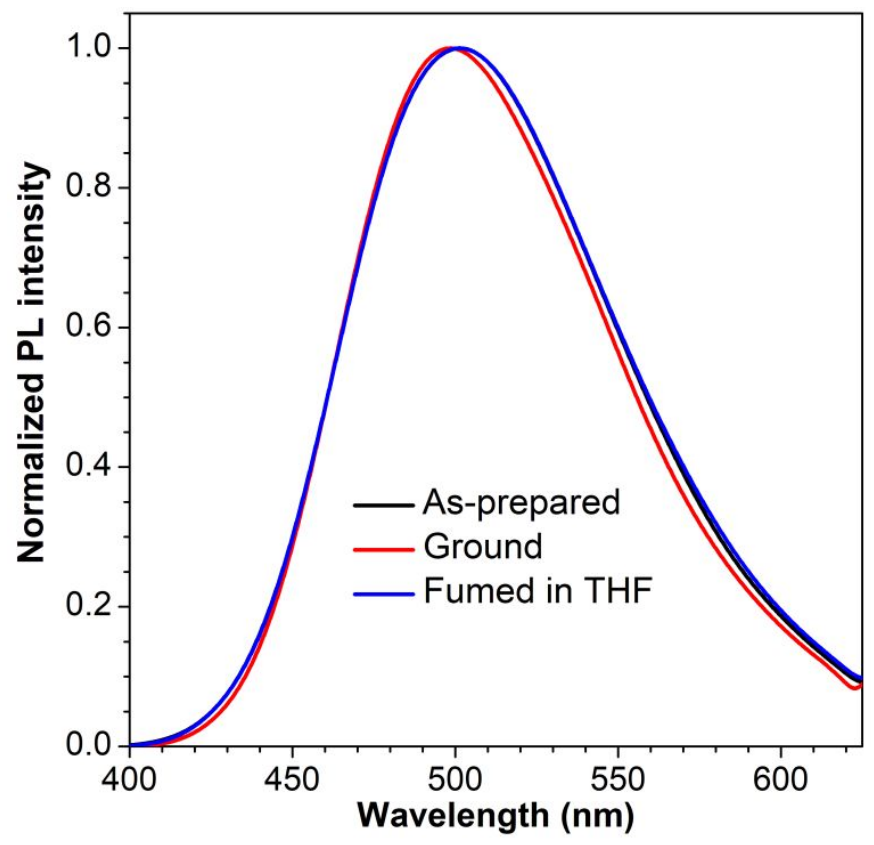

Figure S25. PL spectra of as-prepared samples of acyclic 7 precursor after grinding and subsequent solvent fuming with THF for 30 minutes.

(a)

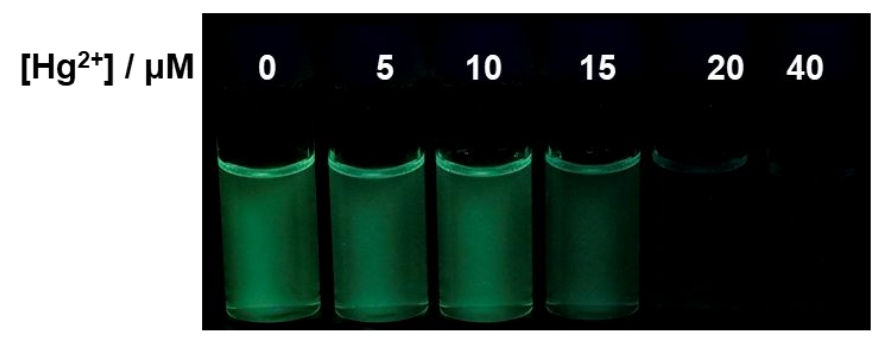

(b)

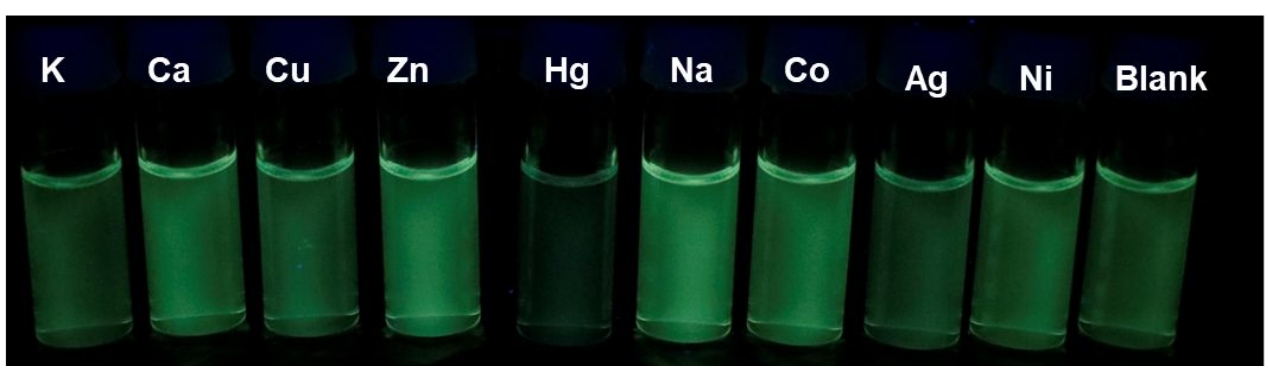

Figure S26. (a) Fluorescent images of the solution of TPEMCS $(5 \mu \mathrm{M})$ in the THF/water mixtures at $f_{w}=90 \%$ with different concentration of $\mathrm{Hg}^{2+}$ ions under UV irradiation. (b) Fluorescent images of the solution of TPEMCS $(5 \mu \mathrm{M})$ in the THF/water mixtures at $f_{w}=90 \%$ with different metal ions $(20 \mu \mathrm{M})$ under UV irradiation. 

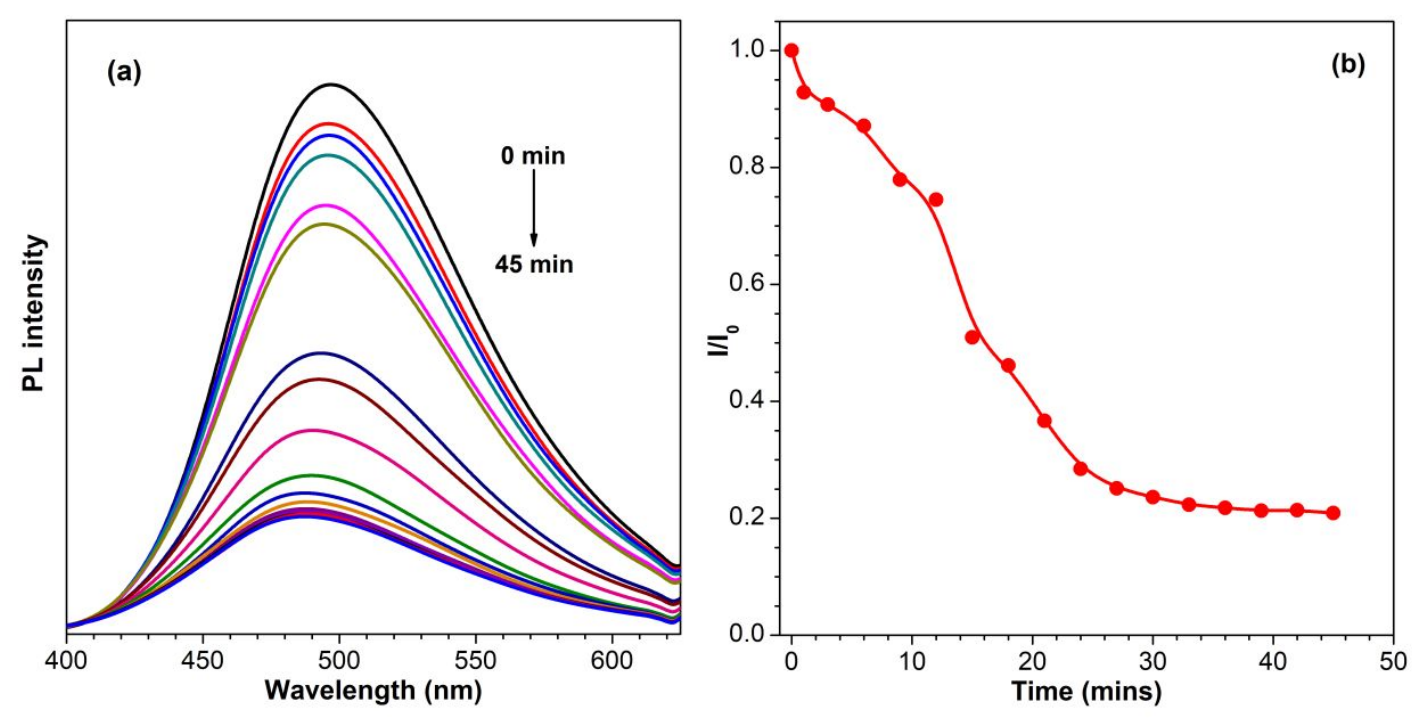

Figure S27. (a) Fluorescent spectra of the solution of TPEMCS $(5 \mu \mathrm{M})$ in the THF/water mixtures at $f_{w}=90 \%$ after addition of $\mathrm{Hg}^{2+}$ ions $(20 \mu \mathrm{M})$ within 45 minutes. (b) Change of PL intensity of TPEMCS samples with time after the addition of $\mathrm{Hg}^{2+}$ ions $(20 \mu \mathrm{M})$.
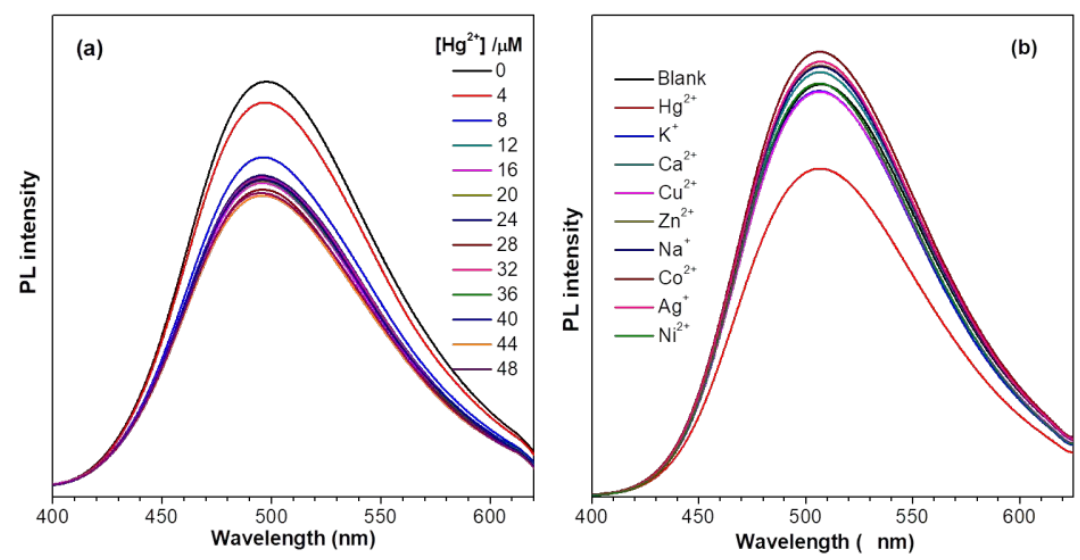

(c)

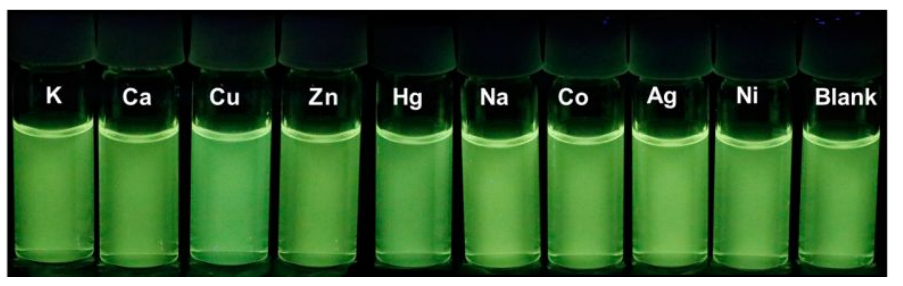

Figure S28. (a) PL spectra of the solution of TPEMC $(5 \mu \mathrm{M})$ in the THF/water mixtures at $f_{w}=$ $90 \%$ with different concentration of $\mathrm{Hg}^{2+}$ ions. (b) PL spectra of the solution of TPEMC (5 $\left.\mu \mathrm{M}\right)$ in the THF/water mixtures at $f_{w}=90 \%$ with different metal ions $(20 \mu \mathrm{M})$. (c) Fluorescent images of the solution of TPEMC ( $5 \mu \mathrm{M})$ in the THF/water mixtures at $f_{w}=90 \%$ with different metal ions $(20 \mu \mathrm{M})$ under UV irradiation. 

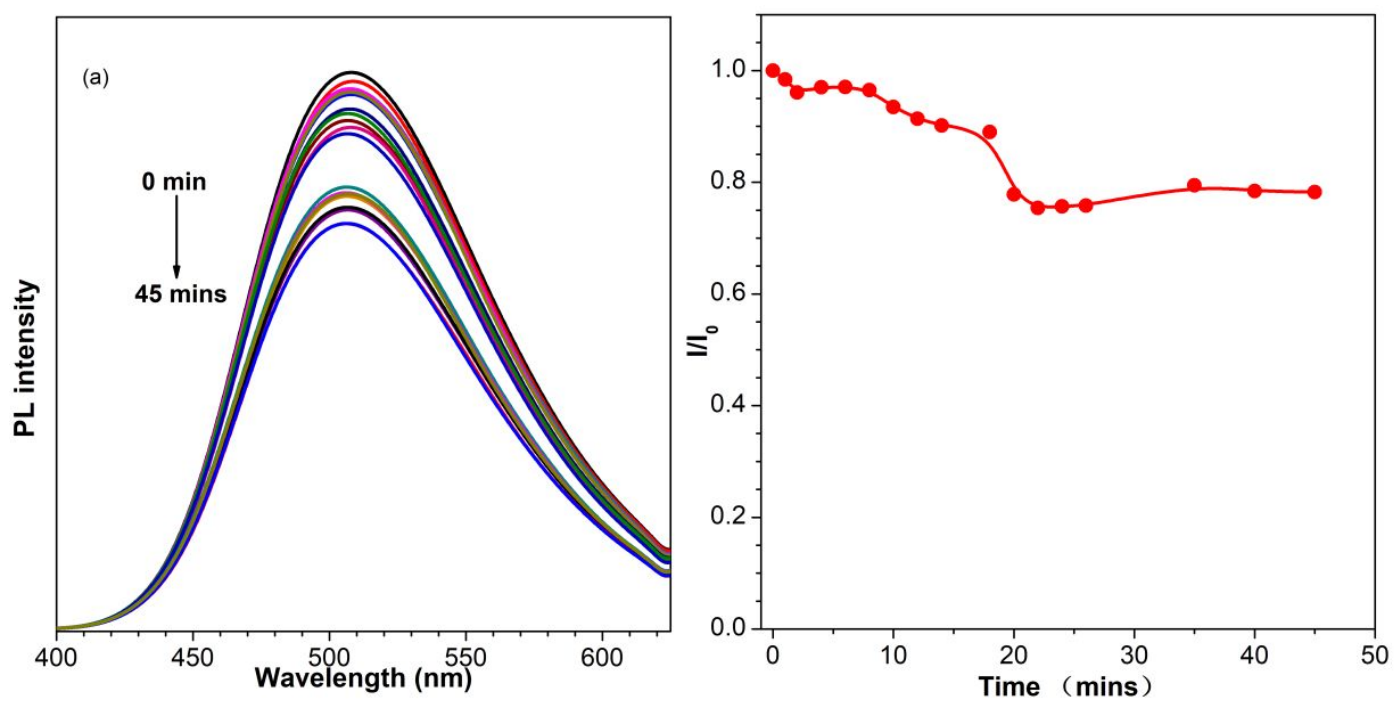

Figure S29. (a) Fluorescent spectra of the solution of TPEMC $(5 \mu \mathrm{M})$ in the THF/water mixtures at $f_{w}=90 \%$ after addition of $\mathrm{Hg}^{2+}$ ions $(20 \mu \mathrm{M})$ within 45 minutes. (b) Change of PL intensity of TPEMC samples with time after the addition of $\mathrm{Hg}^{2+}$ ions $(20 \mu \mathrm{M})$. 


\section{NMR spectra of synthetic intermediates and products}

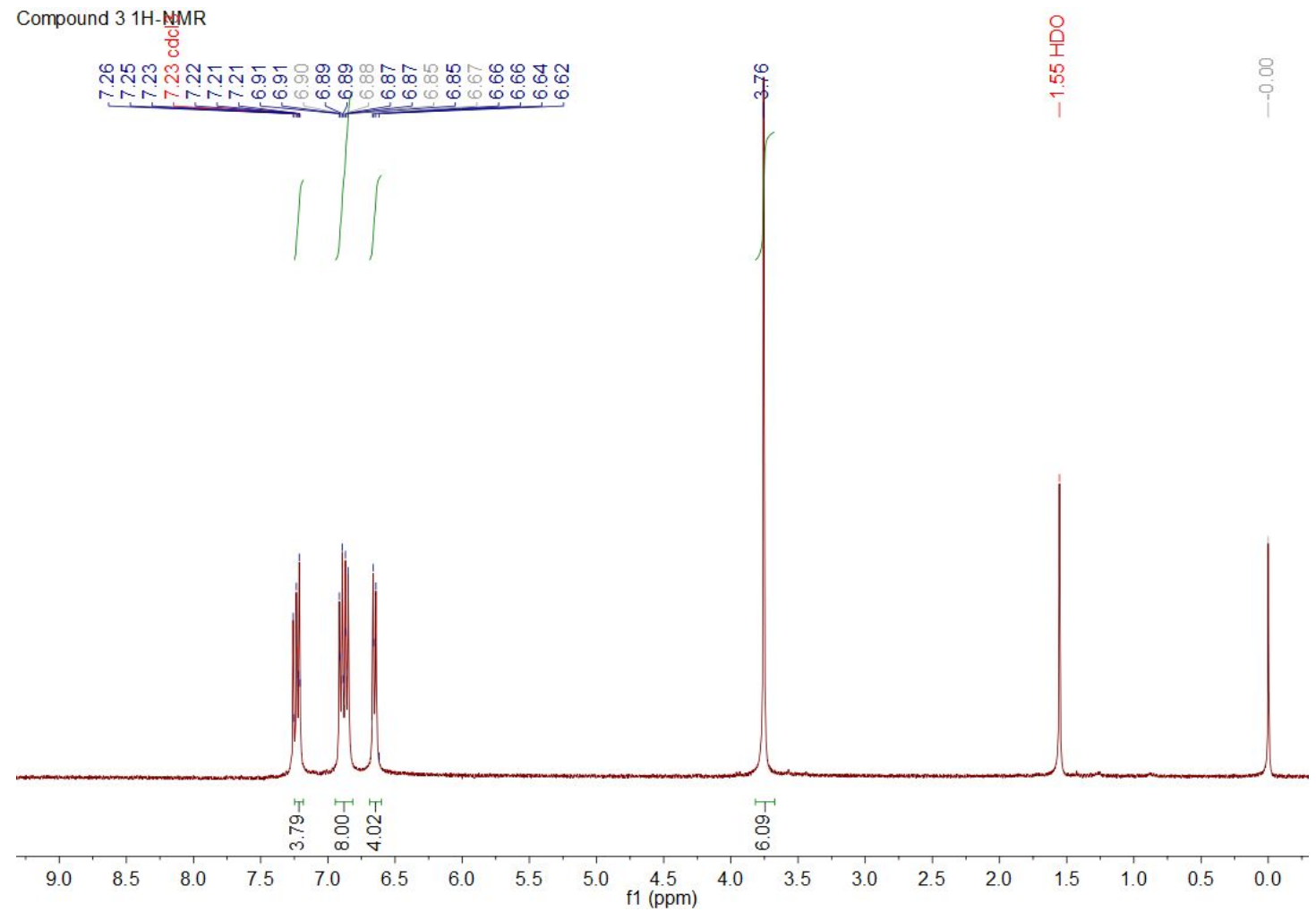

Figure S30. ${ }^{1} \mathrm{H}$ NMR spectrum of compound 3 in $\mathrm{CDCl}_{3}$ at $298 \mathrm{~K}$.

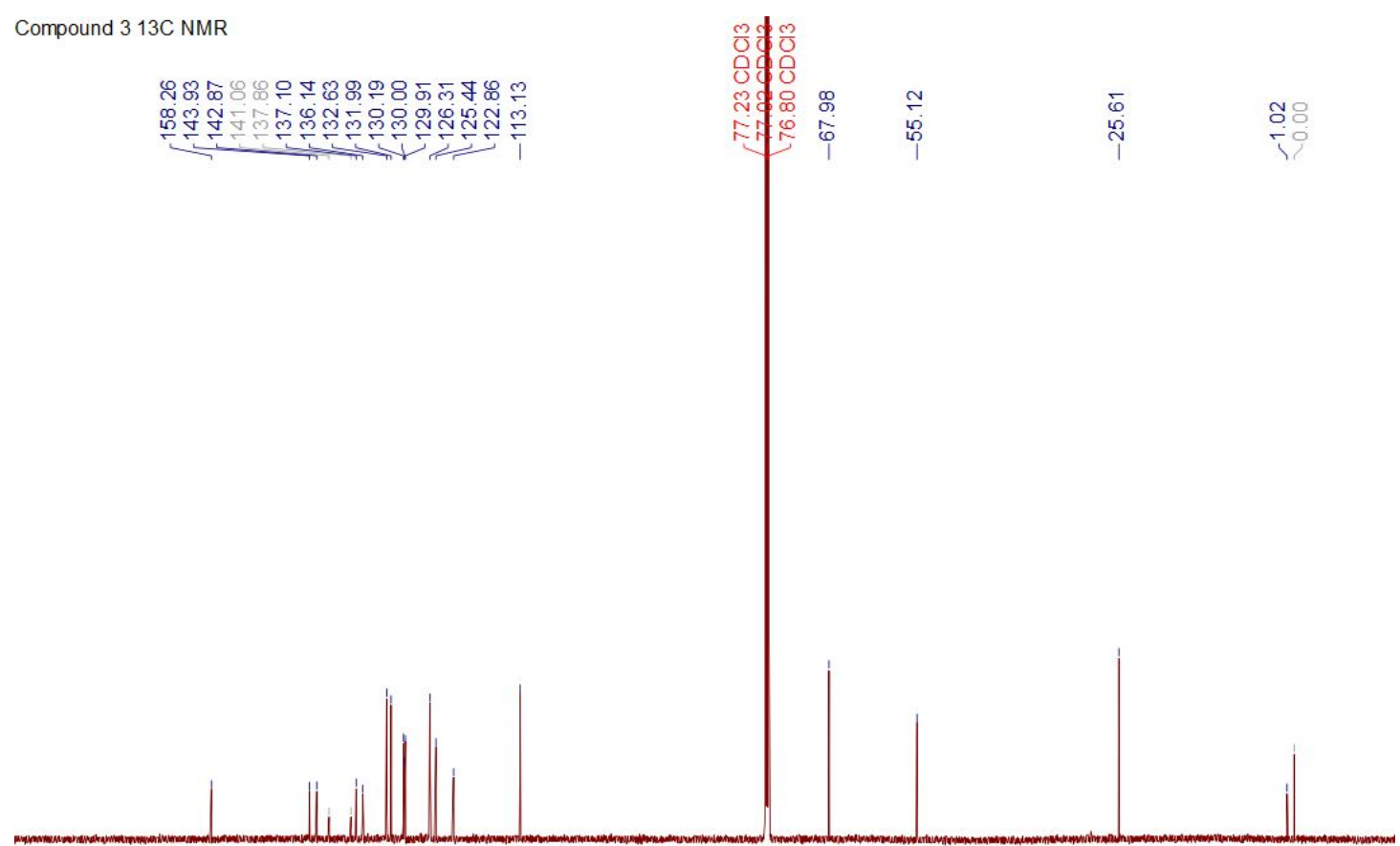

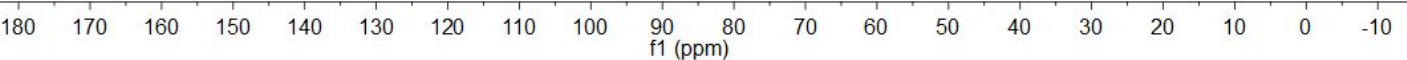

Figure S31. ${ }^{13} \mathrm{C}$ NMR spectrum of compound $\mathbf{3}$ in $\mathrm{CDCl}_{3}$ at $298 \mathrm{~K}$. 


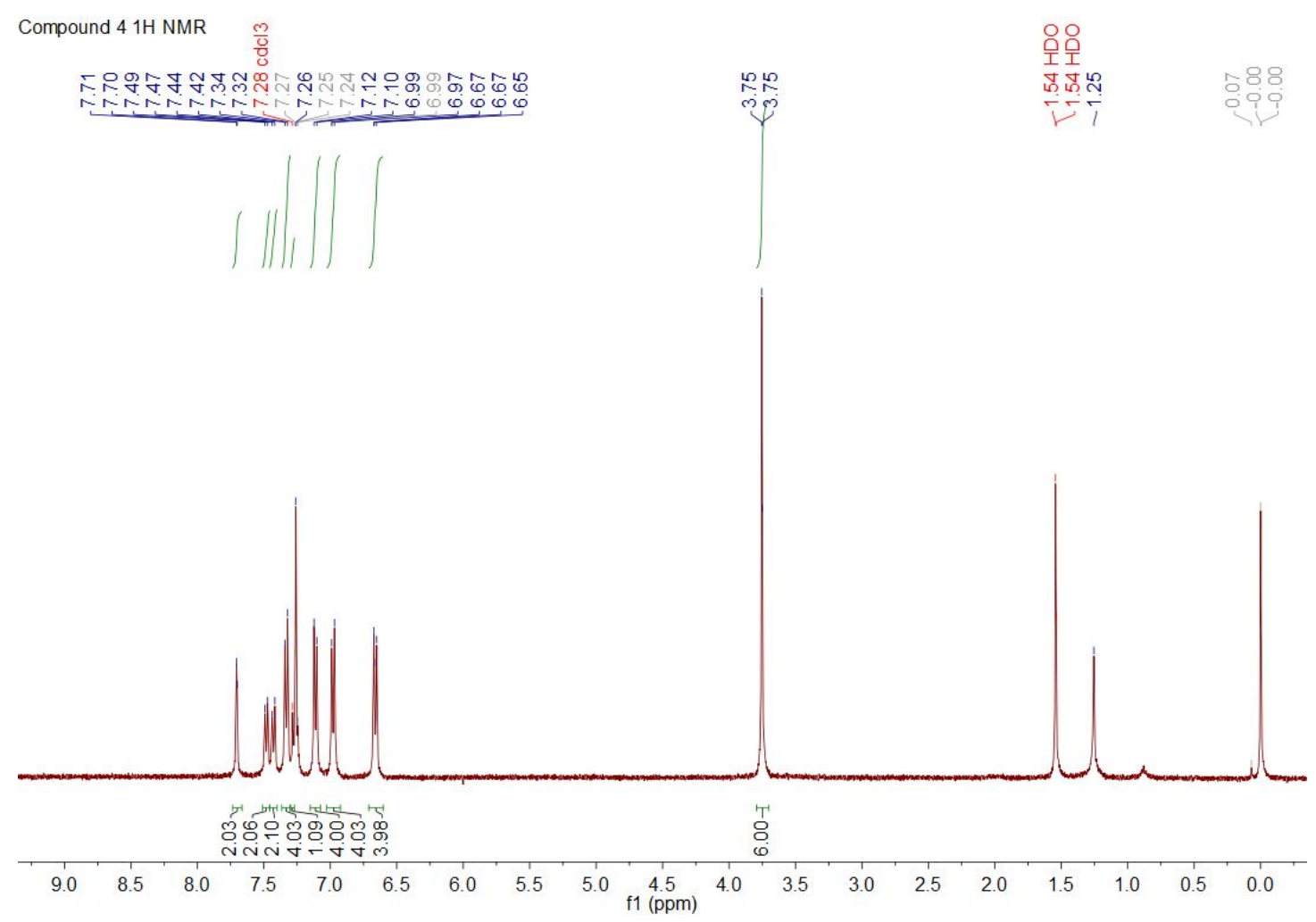

Figure S32. ${ }^{1} \mathrm{H}$ NMR spectrum of compound 4 in $\mathrm{CDCl}_{3}$ at $298 \mathrm{~K}$.

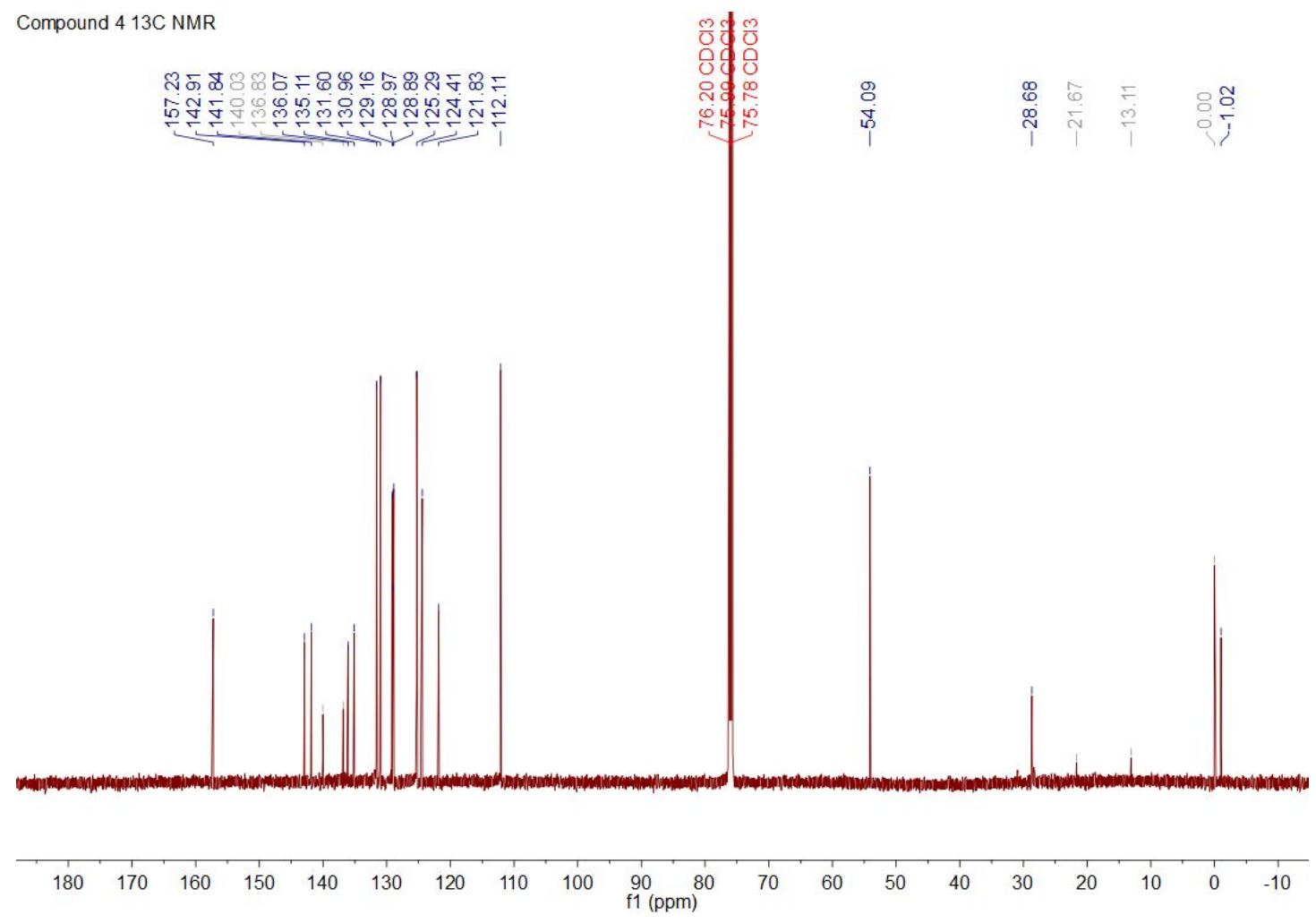

Figure S33. ${ }^{13} \mathrm{C}$ NMR spectrum of compound 4 in $\mathrm{CDCl}_{3}$ at $298 \mathrm{~K}$. 


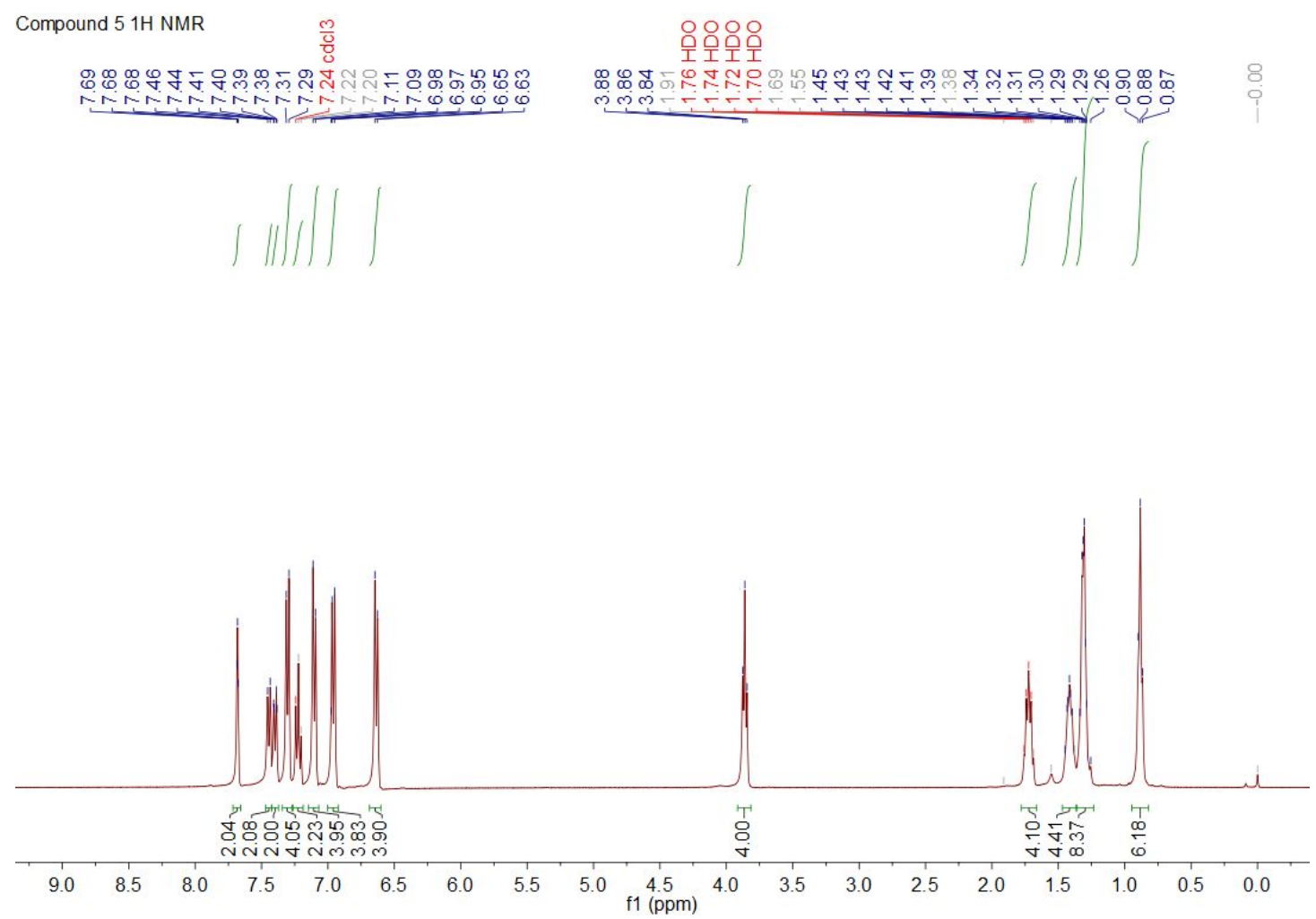

Figure S34. ${ }^{1} \mathrm{H}$ NMR spectrum of compound $\mathbf{5}$ in $\mathrm{CDCl}_{3}$ at $298 \mathrm{~K}$.

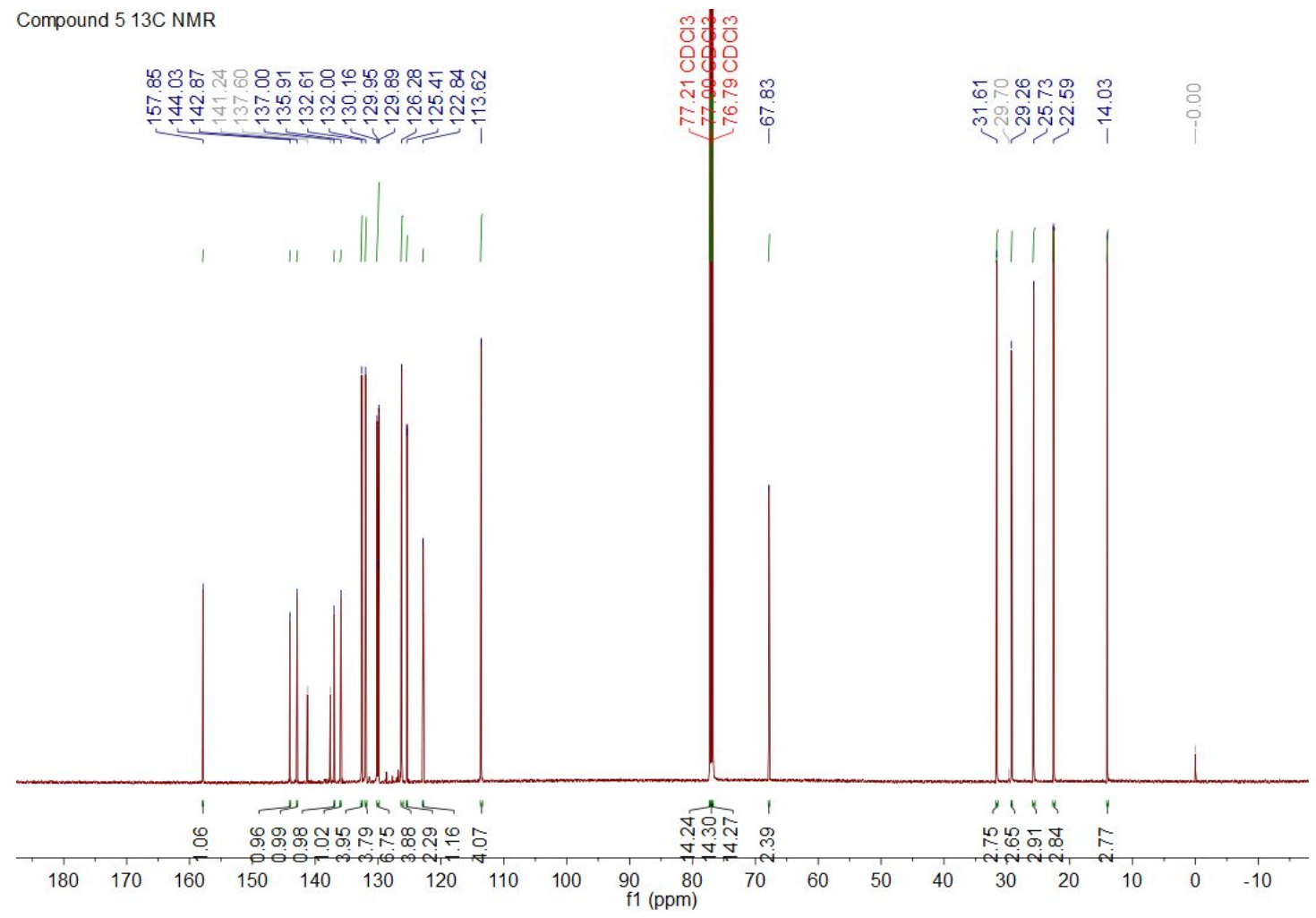

Figure S35. ${ }^{13} \mathrm{C}$ NMR spectrum of compound $\mathbf{5}$ in $\mathrm{CDCl}_{3}$ at $298 \mathrm{~K}$. 


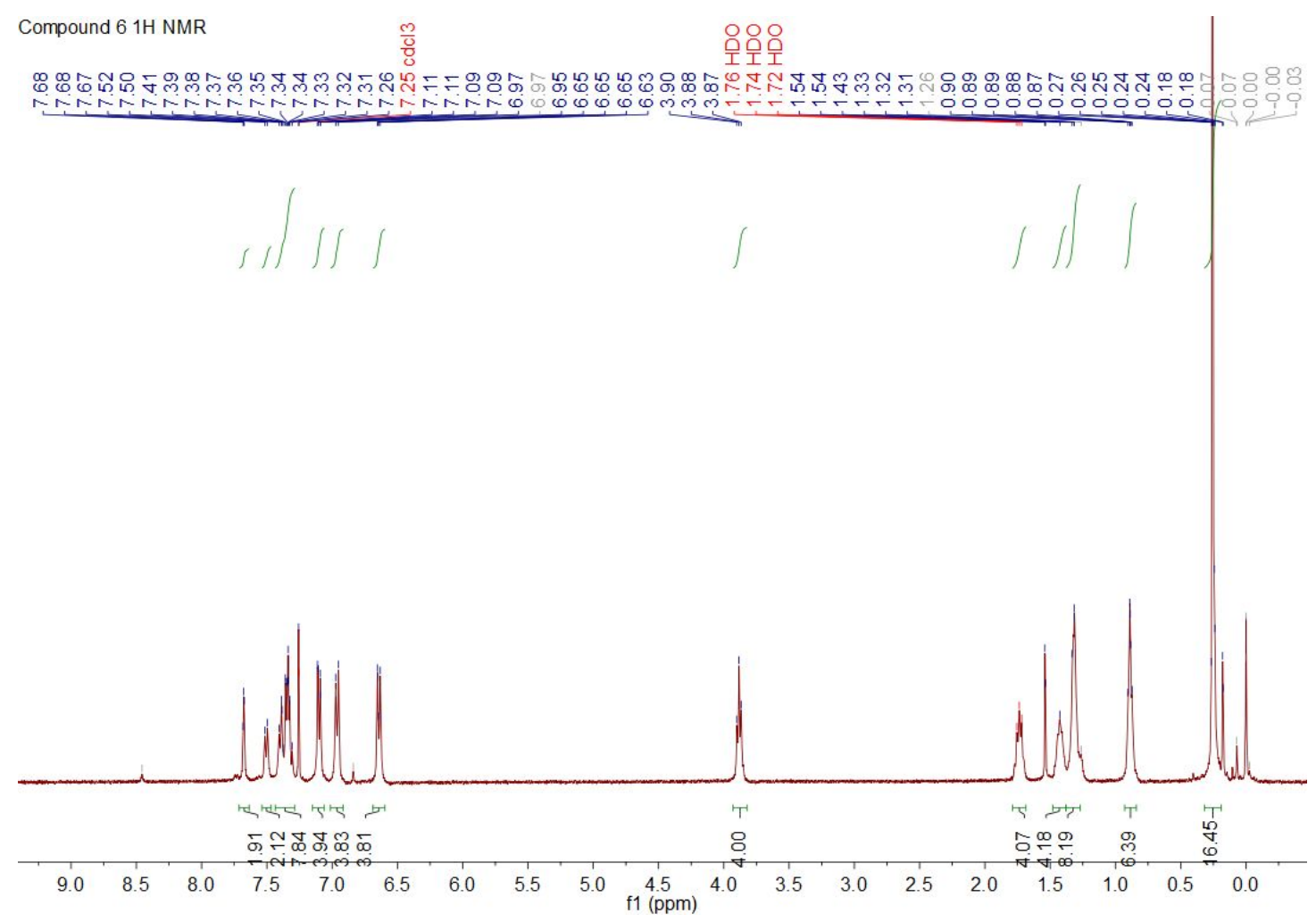

Figure S36. ${ }^{1} \mathrm{H}$ NMR spectrum of compound 6 in $\mathrm{CDCl}_{3}$ at $298 \mathrm{~K}$.

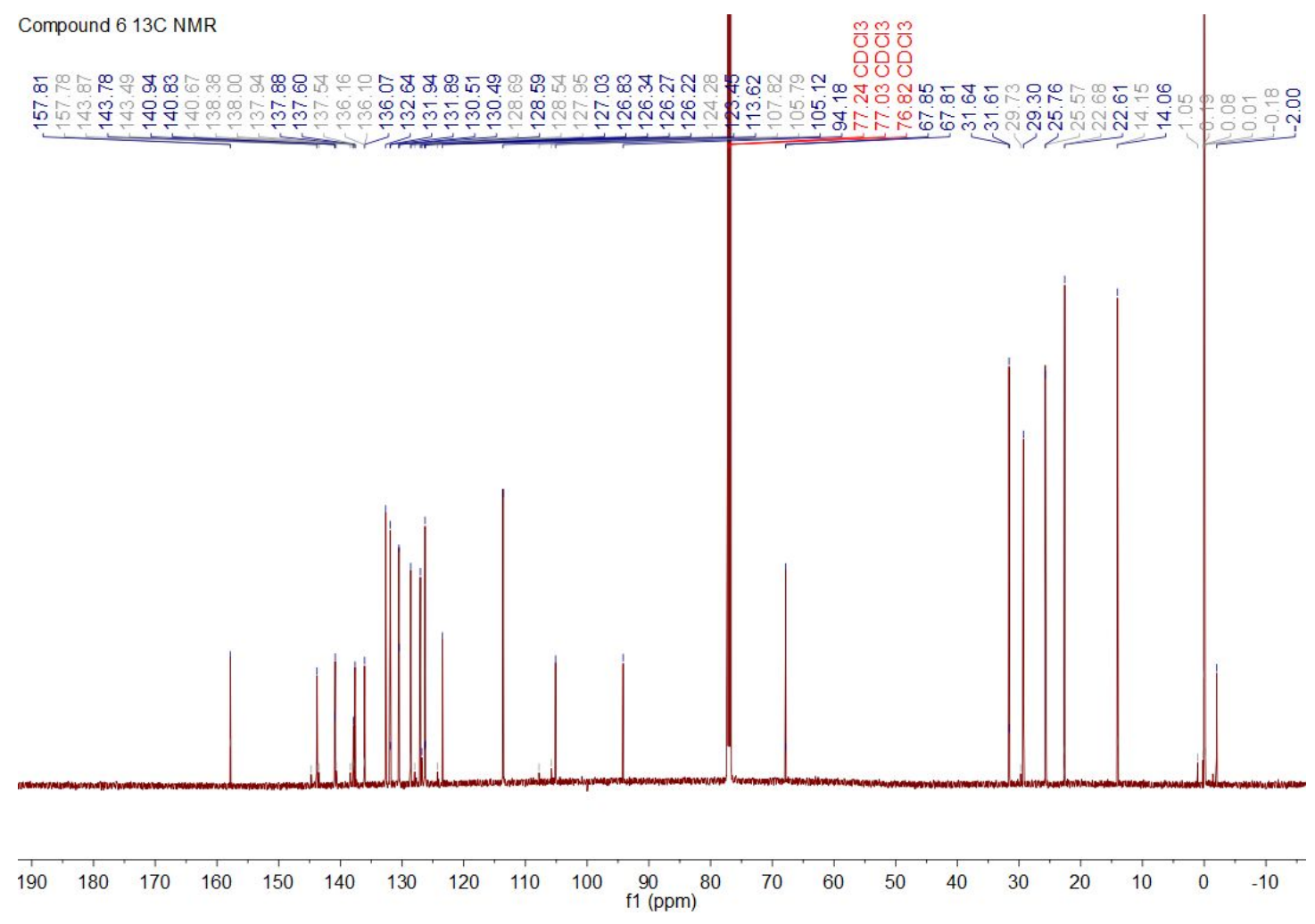

Figure S37. ${ }^{13} \mathrm{C}$ NMR spectrum of compound 6 in $\mathrm{CDCl}_{3}$ at $298 \mathrm{~K}$. 

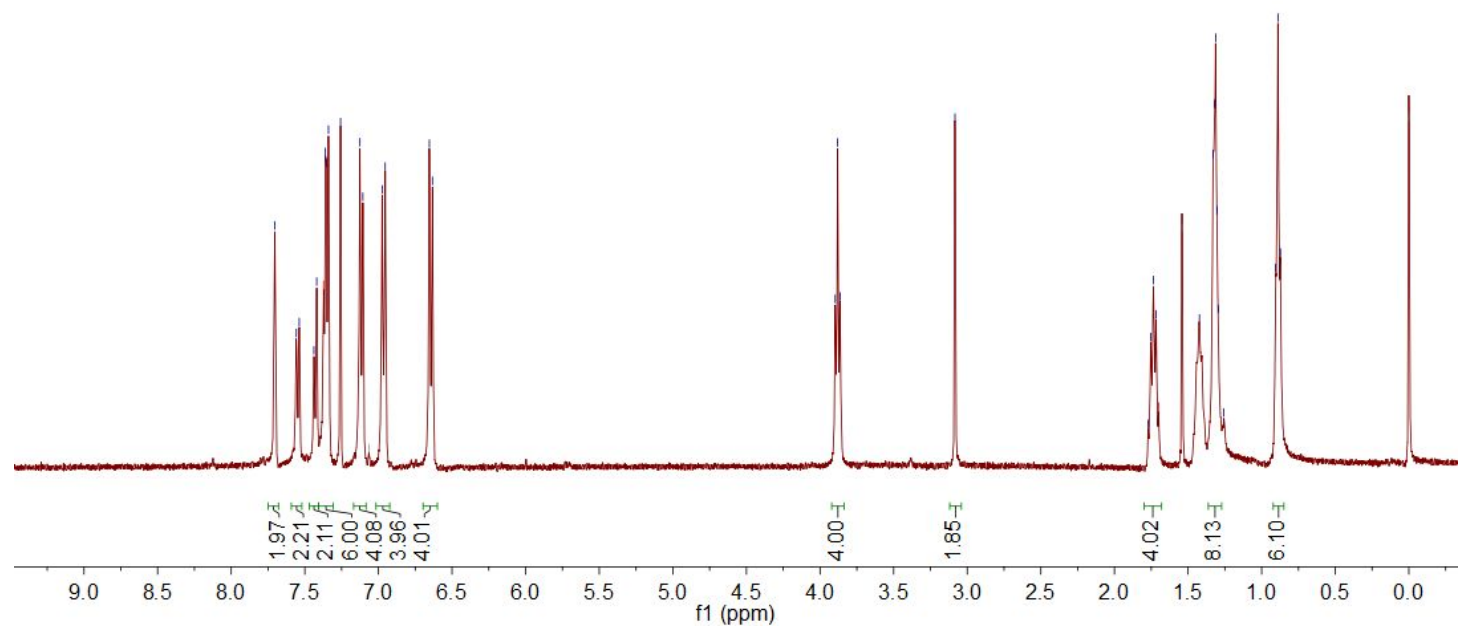

Figure S38. ${ }^{1} \mathrm{H}$ NMR spectrum of acyclic precursor 7 in $\mathrm{CDCl}_{3}$ at $298 \mathrm{~K}$.

Compound 7 13C NMR

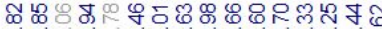

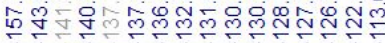

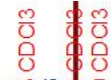

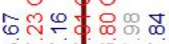

MNN 0 -

OND

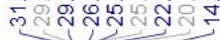

$\int$
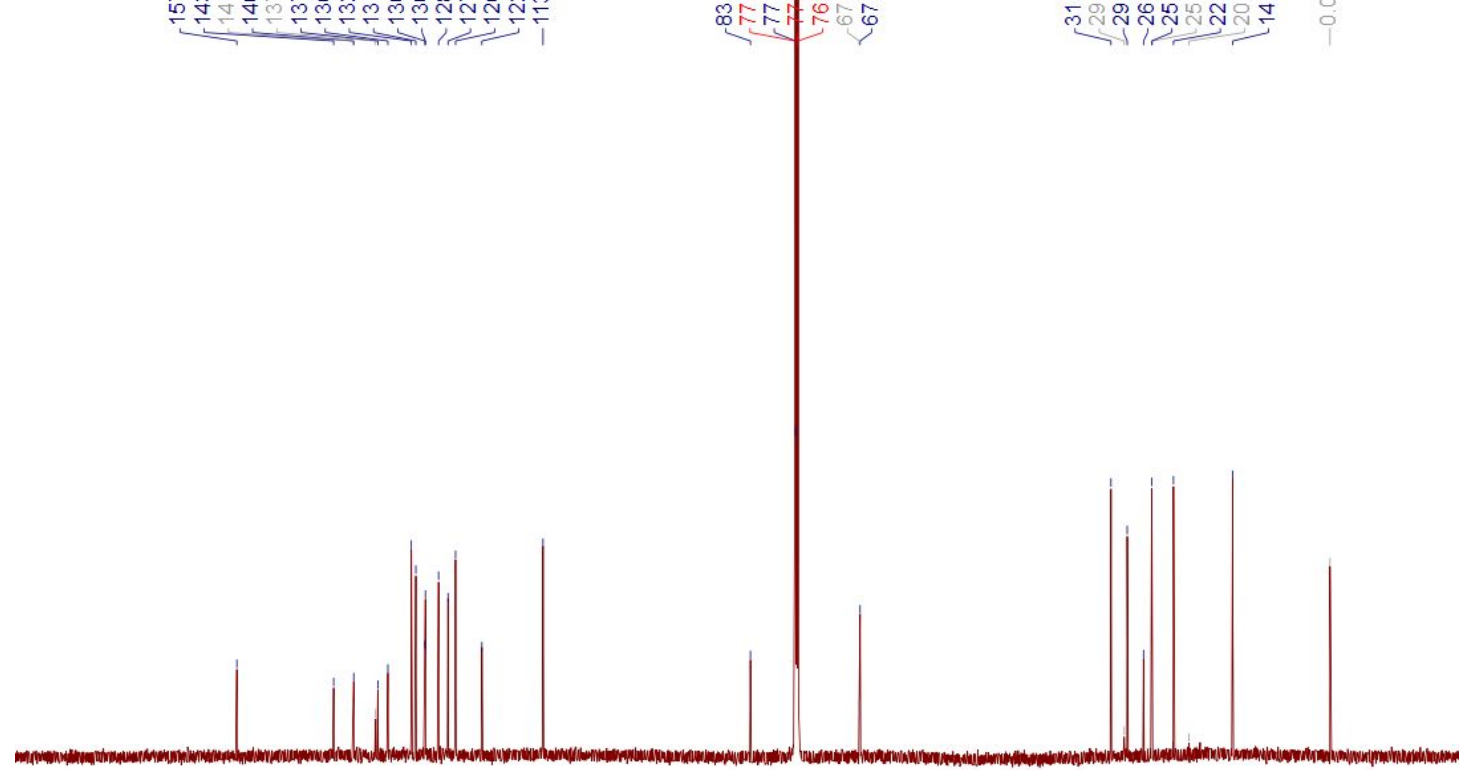

$\begin{array}{lllllllllll}180 & 170 & 160 & 150 & 140 & 130 & 120 & 110 & 100 & 90 & 80\end{array}$

Figure S39. ${ }^{13} \mathrm{C}$ NMR spectrum of acyclic precursor 7 in $\mathrm{CDCl}_{3}$ at $298 \mathrm{~K}$. 


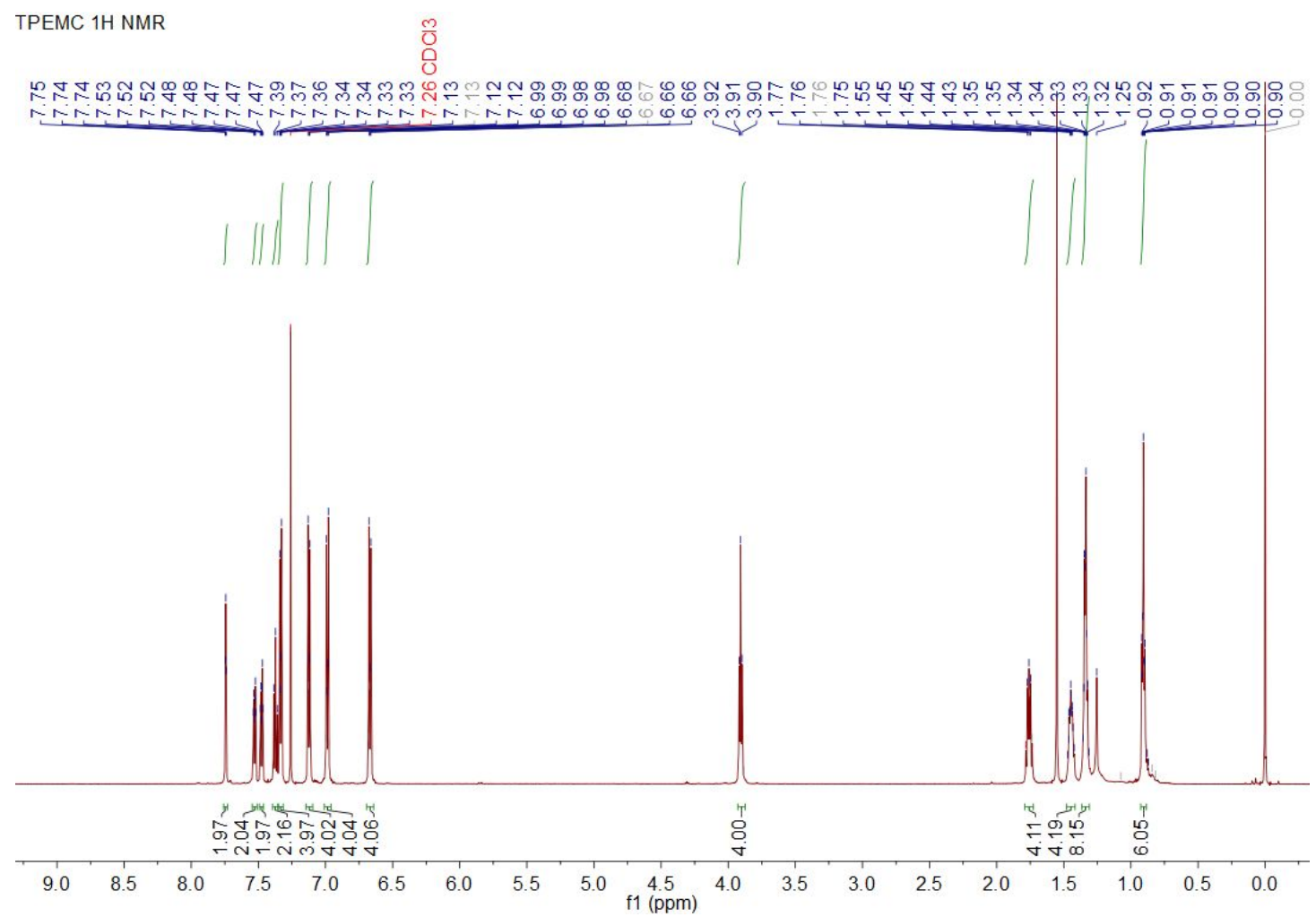

Figure S40. ${ }^{1} \mathrm{H}$ NMR spectrum of macrocyclic luminogen TPEMC in $\mathrm{CDCl}_{3}$ at $298 \mathrm{~K}$.

TPEMC 13C NMR

$\begin{array}{lllllllllll}190 & 180 & 170 & 160 & 150 & 140 & 130 & 120 & 110 & 100 & 90\end{array}$

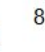

8070

Figure S41. ${ }^{13} \mathrm{C}$ NMR spectrum of macrocyclicluminogen TPEMC in $\mathrm{CDCl}_{3}$ at $298 \mathrm{~K}$. 


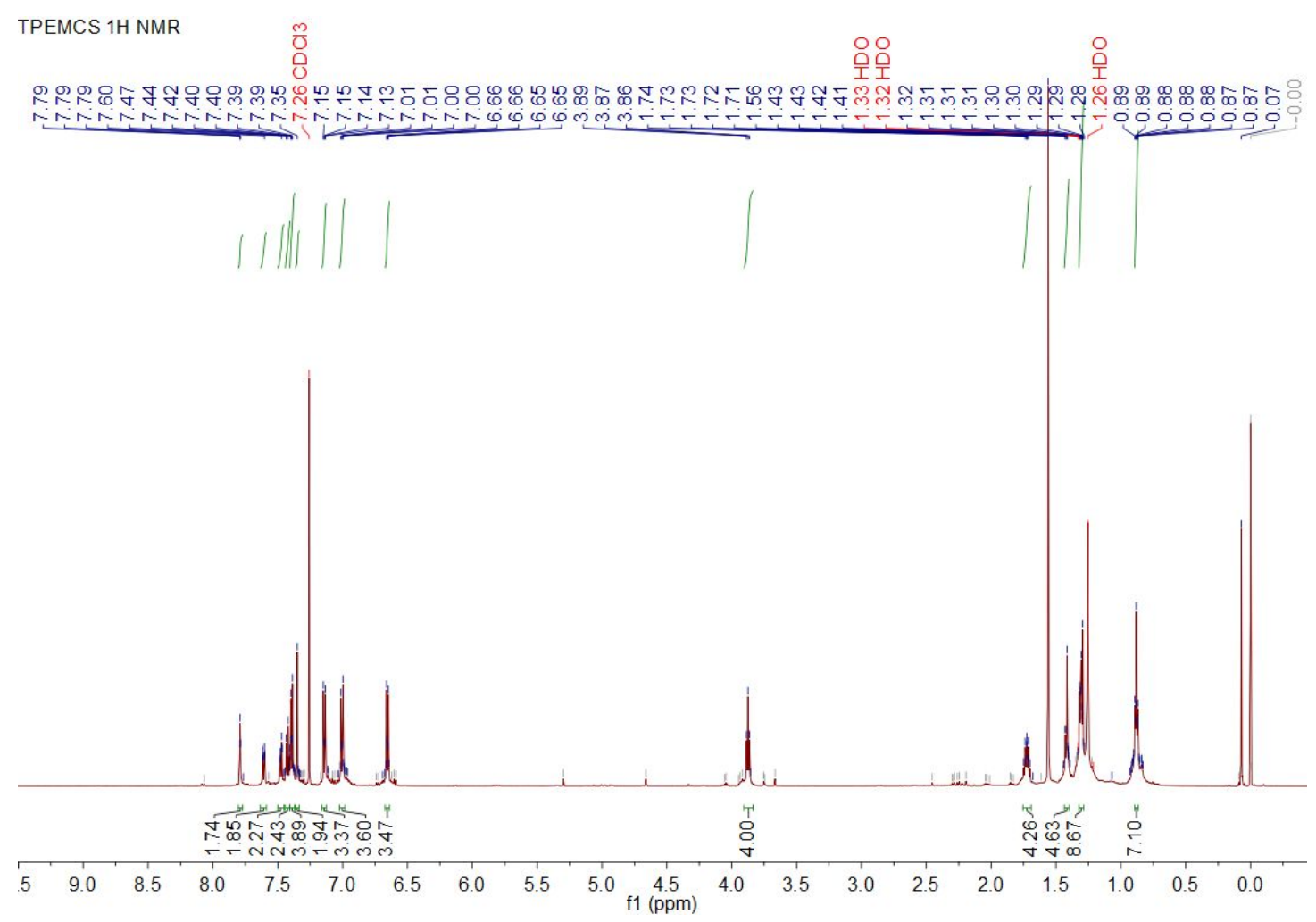

Figure S42. ${ }^{1} \mathrm{H}$ NMR spectrum of macrocyclicluminogen TPEMCS in $\mathrm{CDCl}_{3}$ at $298 \mathrm{~K}$.

TPEMCS 13C NMR

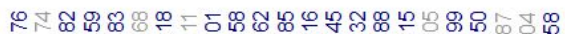

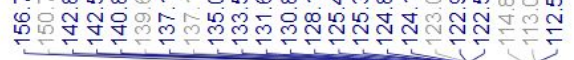

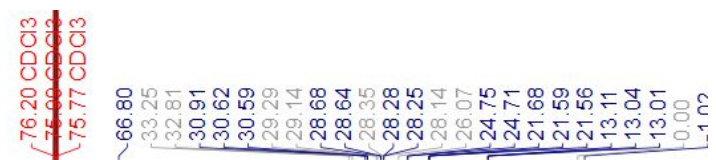

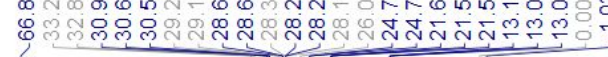

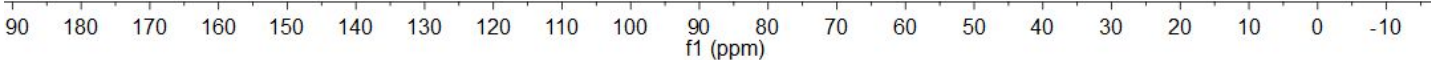

Figure S43. ${ }^{13} \mathrm{C}$ NMR spectrum of macrocyclicluminogen TPEMCS in $\mathrm{CDCl}_{3}$ at $298 \mathrm{~K}$. 


\section{Density functional theory (DFT) calculation}

Gaussian 09 program ${ }^{[1]}$ was used for the Density Function Theory (DFT) calculation. Molecular geometries were optimized and energy of each optimized conformer was calculated at the B3LYP level of DFT with the 6-31G(d) basis set.

\section{Optimized Cartesian coordinates of TPEMC at B3LYP/6-31G* level of theory}

\section{(M,M)-TPEMC}

\begin{tabular}{|c|c|c|c|}
\hline $\mathrm{C}$ & -7.28228467 & 3.27398004 & -1.43735937 \\
\hline $\mathrm{C}$ & -6.21601421 & 3.58220257 & -0.57526422 \\
\hline $\mathrm{C}$ & -5.94529094 & 2.68537859 & 0.47190334 \\
\hline $\mathrm{C}$ & -6.70908390 & 1.53632582 & 0.65071637 \\
\hline $\mathrm{C}$ & -7.79468441 & 1.24282978 & -0.19308923 \\
\hline $\mathrm{C}$ & -8.05415007 & 2.13230147 & -1.24997708 \\
\hline $\mathrm{C}$ & -5.40677345 & 4.81247599 & -0.76365650 \\
\hline $\mathrm{C}$ & -8.60018204 & -0.00000052 & -0.00001709 \\
\hline $\mathrm{C}$ & -7.79467541 & -1.24282519 & 0.19305479 \\
\hline $\mathrm{C}$ & -9.96918660 & -0.00000517 & -0.00000670 \\
\hline $\mathrm{C}$ & -6.70908353 & -1.53632380 & -0.65076078 \\
\hline $\mathrm{C}$ & -5.94528681 & -2.68537437 & -0.47194909 \\
\hline $\mathrm{C}$ & -6.21599643 & -3.58219209 & 0.57522752 \\
\hline $\mathrm{C}$ & -7.28225790 & -3.27396624 & 1.43733249 \\
\hline $\mathrm{C}$ & -8.05412837 & -2.13229087 & 1.24995057 \\
\hline $\mathrm{C}$ & -5.40675138 & -4.81246288 & 0.76361716 \\
\hline $\mathrm{C}$ & -10.77278977 & -1.25409341 & -0.08985891 \\
\hline $\mathrm{C}$ & -10.77279374 & 1.25407917 & 0.08986869 \\
\hline $\mathrm{C}$ & -10.50151833 & 2.23765600 & 1.05115390 \\
\hline $\mathrm{C}$ & -11.27150308 & 3.39705152 & 1.15626939 \\
\hline $\mathrm{C}$ & -12.34586440 & 3.59695795 & 0.28157717 \\
\hline $\mathrm{C}$ & -12.64214635 & 2.61953983 & -0.68063560 \\
\hline $\mathrm{C}$ & -11.87502433 & 1.46695487 & -0.76257945 \\
\hline $\mathrm{C}$ & -11.87500465 & -1.46696669 & 0.76261066 \\
\hline $\mathrm{C}$ & -12.64212470 & -2.61955457 & 0.68068922 \\
\hline $\mathrm{C}$ & -12.34585704 & -3.59697913 & -0.28152130 \\
\hline $\mathrm{C}$ & -11.27151260 & -3.39707506 & -1.15623500 \\
\hline $\mathrm{C}$ & -10.50152949 & -2.23767670 & -1.05114209 \\
\hline $\mathrm{C}$ & -6.00247950 & 6.00717801 & -1.20675095 \\
\hline $\mathrm{C}$ & -5.24512189 & 7.16581371 & -1.37656914 \\
\hline $\mathrm{C}$ & -3.88061475 & 7.16551780 & -1.10546965 \\
\hline $\mathrm{C}$ & -3.25763634 & 5.98240034 & -0.65930016 \\
\hline $\mathrm{C}$ & -4.03108389 & 4.81729235 & -0.49774197 \\
\hline $\mathrm{C}$ & -4.03106778 & -4.81728214 & 0.49767149 \\
\hline $\mathrm{C}$ & -3.25761672 & -5.98238875 & 0.65922349 \\
\hline
\end{tabular}




\begin{tabular}{|c|c|c|c|}
\hline $\mathrm{C}$ & -3.88058536 & -7.16550218 & 1.10541714 \\
\hline $\mathrm{C}$ & -5.24508634 & -7.16579508 & 1.37654759 \\
\hline $\mathrm{C}$ & -6.00244764 & -6.00716097 & 1.20673541 \\
\hline $\mathrm{C}$ & -1.86295090 & 5.96448219 & -0.37984416 \\
\hline $\mathrm{C}$ & -1.86293927 & -5.96447536 & 0.37972675 \\
\hline $\mathrm{C}$ & -0.66617179 & 5.95994682 & -0.13607612 \\
\hline $\mathrm{C}$ & -0.66616800 & -5.95994529 & 0.13591951 \\
\hline $\mathrm{C}$ & 7.28223863 & -3.27393774 & -1.43738915 \\
\hline $\mathrm{C}$ & 6.21599934 & -3.58218497 & -0.57526399 \\
\hline $\mathrm{C}$ & 5.94531248 & -2.68538950 & 0.47193701 \\
\hline $\mathrm{C}$ & 6.70911327 & -1.53634261 & 0.65075604 \\
\hline $\mathrm{C}$ & 7.79468737 & -1.24282544 & -0.19307627 \\
\hline $\mathrm{C}$ & 8.05411197 & -2.13226570 & -1.25000137 \\
\hline $\mathrm{C}$ & 5.40674815 & -4.81245075 & -0.76366326 \\
\hline $\mathrm{C}$ & 8.60018997 & 0.00000160 & 0.00000371 \\
\hline $\mathrm{C}$ & 7.79467891 & 1.24282413 & 0.19307725 \\
\hline $\mathrm{C}$ & 9.96919676 & 0.00000474 & 0.00001645 \\
\hline $\mathrm{C}$ & 6.70911618 & 1.53634140 & -0.65076961 \\
\hline $\mathrm{C}$ & 5.94531068 & 2.68538630 & -0.47195788 \\
\hline $\mathrm{C}$ & 6.21598018 & 3.58217873 & 0.57525031 \\
\hline $\mathrm{C}$ & 7.28220762 & 3.27393094 & 1.43738990 \\
\hline $\mathrm{C}$ & 8.05408657 & 2.13226147 & 1.25000883 \\
\hline $\mathrm{C}$ & 5.40672397 & 4.81244241 & 0.76364051 \\
\hline $\mathrm{C}$ & 10.77280670 & 1.25408665 & -0.08987148 \\
\hline $\mathrm{C}$ & 10.77280782 & -1.25407500 & 0.08992743 \\
\hline $\mathrm{C}$ & 11.87501576 & -1.46698598 & -0.76254146 \\
\hline $\mathrm{C}$ & 12.64214312 & -2.61956530 & -0.68056778 \\
\hline $\mathrm{C}$ & 12.34589234 & -3.59693987 & 0.28169851 \\
\hline $\mathrm{C}$ & 11.27155780 & -3.39699497 & 1.15641485 \\
\hline $\mathrm{C}$ & 10.50156685 & -2.23760653 & 1.05126920 \\
\hline $\mathrm{C}$ & 11.87499608 & 1.46699540 & 0.76262229 \\
\hline $\mathrm{C}$ & 12.64212386 & 2.61957604 & 0.68067075 \\
\hline $\mathrm{C}$ & 12.34589281 & 3.59695489 & -0.28159717 \\
\hline $\mathrm{C}$ & 11.27157785 & 3.39701213 & -1.15633830 \\
\hline $\mathrm{C}$ & 10.50158606 & 2.23762242 & -1.05121492 \\
\hline $\mathrm{C}$ & 6.00243547 & -6.00714366 & -1.20680823 \\
\hline $\mathrm{C}$ & 5.24506839 & -7.16577222 & -1.37663041 \\
\hline $\mathrm{C}$ & 3.88057045 & -7.16547821 & -1.10548452 \\
\hline $\mathrm{C}$ & 3.25761072 & -5.98236997 & -0.65926532 \\
\hline $\mathrm{C}$ & 4.03106794 & -4.81726939 & -0.49770075 \\
\hline $\mathrm{C}$ & 4.03104956 & 4.81726129 & 0.49764793 \\
\hline $\mathrm{C}$ & 3.25758810 & 5.98236076 & 0.65920037 \\
\hline $\mathrm{C}$ & 3.88053754 & 7.16546795 & 1.10543687 \\
\hline $\mathrm{C}$ & 5.24502946 & 7.16576148 & 1.37661306 \\
\hline
\end{tabular}




\begin{tabular}{|c|c|c|c|}
\hline $\mathrm{C}$ & 6.00240086 & 6.00713398 & 1.20680313 \\
\hline $\mathrm{C}$ & 1.86293569 & -5.96445240 & -0.37975780 \\
\hline $\mathrm{C}$ & 1.86292068 & 5.96444478 & 0.37965465 \\
\hline $\mathrm{C}$ & 0.66615594 & 5.95994709 & 0.13581448 \\
\hline $\mathrm{C}$ & 0.66616445 & -5.95995095 & -0.13594926 \\
\hline $\mathrm{O}$ & -13.16258137 & -4.69036163 & -0.28721862 \\
\hline $\mathrm{C}$ & -12.91451843 & -5.70898218 & -1.24282647 \\
\hline $\mathrm{O}$ & -13.16259233 & 4.69033756 & 0.28729760 \\
\hline $\mathrm{C}$ & -12.91451668 & 5.70894927 & 1.24291132 \\
\hline $\mathrm{O}$ & 13.16262217 & 4.69033342 & -0.28731988 \\
\hline $\mathrm{C}$ & 12.91459352 & 5.70891085 & -1.24298248 \\
\hline $\mathrm{O}$ & 13.16262313 & -4.69031717 & 0.28744444 \\
\hline $\mathrm{C}$ & 12.91457505 & -5.70888884 & 1.24310798 \\
\hline $\mathrm{H}$ & -7.49149315 & 3.92252360 & -2.28363751 \\
\hline $\mathrm{H}$ & -5.14214436 & 2.90338056 & 1.17041381 \\
\hline $\mathrm{H}$ & -6.47434389 & 0.86151302 & 1.46908049 \\
\hline $\mathrm{H}$ & -8.86767304 & 1.91778436 & -1.93583433 \\
\hline $\mathrm{H}$ & -6.47435484 & -0.86151640 & -1.46913260 \\
\hline $\mathrm{H}$ & -5.14214841 & -2.90337999 & -1.17046790 \\
\hline $\mathrm{H}$ & -7.49145695 & -3.92250576 & 2.28361609 \\
\hline $\mathrm{H}$ & -8.86764690 & -1.91777273 & 1.93581294 \\
\hline $\mathrm{H}$ & -9.67205939 & 2.09489558 & 1.73663248 \\
\hline $\mathrm{H}$ & -11.02862263 & 4.12678515 & 1.92040591 \\
\hline $\mathrm{H}$ & -13.48230736 & 2.78618682 & -1.34775037 \\
\hline $\mathrm{H}$ & -12.12489432 & 0.71492349 & -1.50547768 \\
\hline $\mathrm{H}$ & -12.12486389 & -0.71493052 & 1.50550750 \\
\hline $\mathrm{H}$ & -13.48227323 & -2.78619903 & 1.34782031 \\
\hline $\mathrm{H}$ & -11.02864475 & -4.12681264 & -1.92037166 \\
\hline $\mathrm{H}$ & -9.67208564 & -2.09491812 & -1.73663903 \\
\hline $\mathrm{H}$ & -7.07205758 & 6.03262503 & -1.39333414 \\
\hline $\mathrm{H}$ & -5.72700226 & 8.07962662 & -1.71355933 \\
\hline $\mathrm{H}$ & -3.28720912 & 8.06522427 & -1.23254734 \\
\hline $\mathrm{H}$ & -3.53739888 & 3.90459829 & -0.18047536 \\
\hline $\mathrm{H}$ & -3.53739016 & -3.90459202 & 0.18038183 \\
\hline $\mathrm{H}$ & -3.28717766 & -8.06520834 & 1.23248713 \\
\hline $\mathrm{H}$ & -5.72695971 & -8.07960498 & 1.71355593 \\
\hline $\mathrm{H}$ & -7.07202181 & -6.03260662 & 1.39334141 \\
\hline $\mathrm{H}$ & 7.49141399 & -3.92245481 & -2.28369565 \\
\hline $\mathrm{H}$ & 5.14218864 & -2.90340938 & 1.17046804 \\
\hline $\mathrm{H}$ & 6.47440114 & -0.86155186 & 1.46914630 \\
\hline $\mathrm{H}$ & 8.86760732 & -1.91772767 & -1.93588437 \\
\hline $\mathrm{H}$ & 6.47441797 & 0.86155335 & -1.46916609 \\
\hline $\mathrm{H}$ & 5.14219695 & 2.90340732 & -1.17050023 \\
\hline $\mathrm{H}$ & 7.49137001 & 3.92244619 & 2.2837010 \\
\hline
\end{tabular}




$\begin{array}{lrrr}\mathrm{H} & 8.86757408 & 1.91772390 & 1.93590143 \\ \mathrm{H} & 12.12486130 & -0.71498863 & -1.50548230 \\ \mathrm{H} & 13.48228434 & -2.78624095 & -1.34770029 \\ \mathrm{H} & 11.02870317 & -4.12669300 & 1.92059356 \\ \mathrm{H} & 9.67212993 & -2.09481628 & 1.73676806 \\ \mathrm{H} & 12.12482664 & 0.71499485 & 1.50556482 \\ \mathrm{H} & 13.48225042 & 2.78624947 & 1.34782229 \\ \mathrm{H} & 11.02873979 & 4.12671271 & -1.92051972 \\ \mathrm{H} & 9.67216597 & 2.09483380 & -1.73673421 \\ \mathrm{H} & 7.07200731 & -6.03258995 & -1.39342660 \\ \mathrm{H} & 5.72693431 & -8.07957846 & -1.71365926 \\ \mathrm{H} & 3.28715780 & -8.06517976 & -1.23256416 \\ \mathrm{H} & 3.53739744 & -3.90458237 & -0.18039123 \\ \mathrm{H} & 3.53738698 & 3.90457587 & 0.18032128 \\ \mathrm{H} & 3.28712212 & 8.06516928 & 1.23250455 \\ \mathrm{H} & 5.72688780 & 8.07956693 & 1.71365493 \\ \mathrm{H} & 7.07196878 & 6.03258042 & 1.39344405 \\ \mathrm{H} & -13.01302239 & -5.33252671 & -2.26967835 \\ \mathrm{H} & -11.91631688 & -6.14848029 & -1.11543408 \\ \mathrm{H} & -13.67120533 & -6.47572975 & -1.06645784 \\ \mathrm{H} & -13.67120967 & 6.47569567 & 1.06656350 \\ \mathrm{H} & -13.01300128 & 5.33248310 & 2.26976110 \\ \mathrm{H} & -11.91631900 & 6.14845251 & 1.11550594 \\ \mathrm{H} & 13.01313039 & 5.33240838 & -2.26981400 \\ \mathrm{H} & 11.91638906 & 6.14841759 & -1.11564317 \\ \mathrm{H} & 13.67127671 & 6.47566439 & -1.06662382 \\ \mathrm{H} & 11.91637387 & -6.14839758 & 1.11574984 \\ \mathrm{H} & 13.01308933 & -5.33237981 & 2.26993925 \\ \mathrm{H} & 13.67126306 & -6.47564249 & 1.06677040 \\ & & & \\ & & & \\ & & \end{array}$

\section{(M,P)-ТРЕМС}

$\begin{array}{lccc}\text { C } & -7.40687998 & -3.41309923 & -1.13260347 \\ \mathrm{C} & -6.29174397 & -3.62672539 & -0.30472997 \\ \mathrm{C} & -5.96615211 & -2.62428263 & 0.62340325 \\ \mathrm{C} & -6.72773570 & -1.46327650 & 0.72397834 \\ \mathrm{C} & -7.85706044 & -1.25743762 & -0.08858772 \\ \mathrm{C} & -8.16852829 & -2.25492647 & -1.02953083 \\ \mathrm{C} & -5.48760713 & -4.86935013 & -0.41792371 \\ \mathrm{C} & -8.65720861 & -0.00002950 & -0.00003894 \\ \mathrm{C} & -7.85705440 & 1.25737934 & 0.08838995 \\ \mathrm{C} & -10.02850483 & -0.00002478 & 0.00010237 \\ \mathrm{C} & -6.72780100 & 1.46319701 & -0.72428234 \\ \mathrm{C} & -5.96621879 & 2.62421319 & -0.62381508 \\ \mathrm{C} & -6.29174355 & 3.62669423 & 0.30430112\end{array}$




\begin{tabular}{|c|c|c|c|}
\hline $\mathrm{C}$ & -7.40681622 & 3.41309647 & 1.13226762 \\
\hline $\mathrm{C}$ & -8.16846093 & 2.25491182 & 1.02930740 \\
\hline $\mathrm{C}$ & -5.48760877 & 4.86932826 & 0.41738031 \\
\hline $\mathrm{C}$ & -10.83075209 & 1.23223629 & -0.25009629 \\
\hline $\mathrm{C}$ & -10.83071071 & -1.23226628 & 0.25054433 \\
\hline $\mathrm{C}$ & -10.54527922 & -2.08495167 & 1.32647461 \\
\hline $\mathrm{C}$ & -11.31136775 & -3.22097346 & 1.59015883 \\
\hline $\mathrm{C}$ & -12.39804058 & -3.53089877 & 0.76379825 \\
\hline $\mathrm{C}$ & -12.70915294 & -2.68453773 & -0.31135237 \\
\hline $\mathrm{C}$ & -11.94478588 & -1.55221184 & -0.55170721 \\
\hline $\mathrm{C}$ & -11.94463247 & 1.55216588 & 0.55242645 \\
\hline $\mathrm{C}$ & -12.70902223 & 2.68453002 & 0.31231399 \\
\hline $\mathrm{C}$ & -12.39812155 & 3.53095829 & -0.76284621 \\
\hline $\mathrm{C}$ & -11.31167038 & 3.22103005 & -1.58949343 \\
\hline $\mathrm{C}$ & -10.54555614 & 2.08496612 & -1.32605638 \\
\hline $\mathrm{C}$ & -6.11298631 & -6.11147488 & -0.62663899 \\
\hline $\mathrm{C}$ & -5.36188943 & -7.28100200 & -0.74075791 \\
\hline $\mathrm{C}$ & -3.97425575 & -7.24057481 & -0.65380393 \\
\hline $\mathrm{C}$ & -3.32116292 & -6.00791245 & -0.44847840 \\
\hline $\mathrm{C}$ & -4.08996283 & -4.83445955 & -0.32847014 \\
\hline $\mathrm{C}$ & -4.08996916 & 4.83444707 & 0.32783857 \\
\hline $\mathrm{C}$ & -3.32117169 & 6.00791023 & 0.44775098 \\
\hline $\mathrm{C}$ & -3.97426132 & 7.24057688 & 0.65306821 \\
\hline $\mathrm{C}$ & -5.36188932 & 7.28099549 & 0.74010722 \\
\hline $\mathrm{C}$ & -6.11298367 & 6.11145667 & 0.62608606 \\
\hline $\mathrm{C}$ & -1.90104480 & -5.95529461 & -0.39166865 \\
\hline $\mathrm{C}$ & -1.90105537 & 5.95530218 & 0.39087574 \\
\hline $\mathrm{C}$ & -0.67976444 & -5.94366441 & -0.37295017 \\
\hline $\mathrm{C}$ & -0.67977524 & 5.94368976 & 0.37212642 \\
\hline $\mathrm{C}$ & 7.40676182 & 3.41295939 & 1.13291193 \\
\hline $\mathrm{C}$ & 6.29175461 & 3.62672018 & 0.30489747 \\
\hline $\mathrm{C}$ & 5.96630208 & 2.62441685 & -0.62343654 \\
\hline $\mathrm{C}$ & 6.72788498 & 1.46341621 & -0.72406017 \\
\hline $\mathrm{C}$ & 7.85707885 & 1.25744336 & 0.08865466 \\
\hline $\mathrm{C}$ & 8.16841769 & 2.25479643 & 1.02978495 \\
\hline $\mathrm{C}$ & 5.48760533 & 4.86932991 & 0.41814536 \\
\hline $\mathrm{C}$ & 8.65722009 & 0.00004182 & 0.00003270 \\
\hline $\mathrm{C}$ & 7.85705112 & -1.25733593 & -0.08875928 \\
\hline $\mathrm{C}$ & 10.02851250 & -0.00000491 & 0.00018336 \\
\hline $\mathrm{C}$ & 6.72773994 & -1.46332285 & 0.72378643 \\
\hline $\mathrm{C}$ & 5.96616608 & -2.62431614 & 0.62301733 \\
\hline $\mathrm{C}$ & 6.29174143 & -3.62659094 & -0.30530701 \\
\hline $\mathrm{C}$ & 7.40686236 & -3.41280634 & -1.13316086 \\
\hline $\mathrm{C}$ & 8.16851527 & -2.25465361 & -1.02988190 \\
\hline
\end{tabular}




\begin{tabular}{|c|c|c|c|}
\hline $\mathrm{C}$ & 5.48760670 & -4.86919639 & -0.41869717 \\
\hline $\mathrm{C}$ & 10.83065938 & -1.23235668 & 0.25027366 \\
\hline $\mathrm{C}$ & 10.83081866 & 1.23227519 & -0.24973397 \\
\hline $\mathrm{C}$ & 11.94472216 & 1.55194847 & 0.55286021 \\
\hline $\mathrm{C}$ & 12.70916807 & 2.68433047 & 0.31302218 \\
\hline $\mathrm{C}$ & 12.39830560 & 3.53103902 & -0.76192908 \\
\hline $\mathrm{C}$ & 11.31183591 & 3.22137081 & -1.58864910 \\
\hline $\mathrm{C}$ & 10.54566319 & 2.08528202 & -1.32548491 \\
\hline $\mathrm{C}$ & 11.94470010 & -1.55214354 & -0.55208199 \\
\hline $\mathrm{C}$ & 12.70899604 & -2.68459056 & -0.31206396 \\
\hline $\mathrm{C}$ & 12.39783315 & -3.53125330 & 0.76283599 \\
\hline $\mathrm{C}$ & 11.31121598 & -3.22147411 & 1.58932000 \\
\hline $\mathrm{C}$ & 10.54520058 & -2.08532088 & 1.32597709 \\
\hline $\mathrm{C}$ & 6.11294165 & 6.11141222 & 0.62723951 \\
\hline $\mathrm{C}$ & 5.36182508 & 7.28092167 & 0.74141282 \\
\hline $\mathrm{C}$ & 3.97421190 & 7.24052126 & 0.65413180 \\
\hline $\mathrm{C}$ & 3.32115938 & 6.00790187 & 0.44841217 \\
\hline $\mathrm{C}$ & 4.08997933 & 4.83446781 & 0.32836207 \\
\hline $\mathrm{C}$ & 4.08997456 & -4.83435438 & -0.32900895 \\
\hline $\mathrm{C}$ & 3.32117343 & -6.00778794 & -0.44918164 \\
\hline $\mathrm{C}$ & 3.97424871 & -7.24038844 & -0.65493930 \\
\hline $\mathrm{C}$ & 5.36186868 & -7.28077027 & -0.74212901 \\
\hline $\mathrm{C}$ & 6.11296673 & -6.11126262 & -0.62782455 \\
\hline $\mathrm{C}$ & 1.90105353 & 5.95530737 & 0.39125267 \\
\hline $\mathrm{C}$ & 1.90106449 & -5.95521375 & -0.39208883 \\
\hline $\mathrm{C}$ & 0.67978765 & -5.94362763 & -0.37310801 \\
\hline $\mathrm{C}$ & 0.67977702 & 5.94369616 & 0.37225012 \\
\hline $\mathrm{O}$ & -13.21275479 & 4.61434071 & -0.92098112 \\
\hline $\mathrm{C}$ & -12.94831079 & 5.50193555 & -1.99567052 \\
\hline $\mathrm{O}$ & -13.21269869 & -4.61422420 & 0.92221358 \\
\hline $\mathrm{C}$ & -12.94790978 & -5.50191428 & 1.99670288 \\
\hline $\mathrm{O}$ & 13.21239685 & -4.61469859 & 0.92090018 \\
\hline $\mathrm{C}$ & 12.94767662 & -5.50254178 & 1.99531644 \\
\hline $\mathrm{O}$ & 13.21300212 & 4.61441252 & -0.91979845 \\
\hline $\mathrm{C}$ & 12.94860146 & 5.50229814 & -1.99425711 \\
\hline $\mathrm{H}$ & -7.65968478 & -4.15025891 & -1.88988911 \\
\hline $\mathrm{H}$ & -5.12073882 & -2.76728077 & 1.29112758 \\
\hline $\mathrm{H}$ & -6.45251538 & -0.70746475 & 1.45400333 \\
\hline $\mathrm{H}$ & -9.01509785 & -2.11130524 & -1.69307774 \\
\hline $\mathrm{H}$ & -6.45263307 & 0.70735898 & -1.45429962 \\
\hline $\mathrm{H}$ & -5.12086120 & 2.76718844 & -1.29161509 \\
\hline $\mathrm{H}$ & -7.65957175 & 4.15028790 & 1.88953890 \\
\hline $\mathrm{H}$ & -9.01498180 & 2.11131507 & 1.69292238 \\
\hline $\mathrm{H}$ & -9.70710950 & -1.85516221 & 1.97699169 \\
\hline
\end{tabular}


H

$\mathrm{H}$

$\mathrm{H}$

$\mathrm{H}$

$\mathrm{H}$

$\mathrm{H}$

$\mathrm{H}$

$\mathrm{H}$

$\mathrm{H}$

$\mathrm{H}$

$\mathrm{H}$

$\mathrm{H}$

$\mathrm{H}$

$\mathrm{H}$

$\mathrm{H}$

$\mathrm{H}$

$\mathrm{H}$

$\mathrm{H}$

$\mathrm{H}$

$\mathrm{H}$

$\mathrm{H}$

$\mathrm{H}$

$\mathrm{H}$

$\mathrm{H}$

$\mathrm{H}$

$\mathrm{H}$

$\mathrm{H}$

$\mathrm{H}$

$\mathrm{H}$

$\mathrm{H}$

$\mathrm{H}$

$\mathrm{H}$

$\mathrm{H}$

$\mathrm{H}$

$\mathrm{H}$

$\mathrm{H}$

$\mathrm{H}$

$\mathrm{H}$

$\mathrm{H}$

$\mathrm{H}$

$\mathrm{H}$

$\mathrm{H}$

$\mathrm{H}$

$\mathrm{H}$

\begin{tabular}{rrc}
-11.05704405 & -3.84657205 & 2.43815788 \\
-13.55834362 & -2.93479679 & -0.93981966 \\
-12.20609233 & -0.90164733 & -1.38137634 \\
-12.20577880 & 0.90155895 & 1.38211285 \\
-13.55807136 & 2.93476231 & 0.94098158 \\
-11.05756159 & 3.84662349 & -2.43756132 \\
-9.70755359 & 1.85517816 & -1.97678878 \\
-7.19695526 & -6.15965890 & -0.67551041 \\
-5.86468121 & -8.23215609 & -0.89351831 \\
-3.38395232 & -8.14648885 & -0.74659895 \\
-3.57991488 & -3.88575731 & -0.19545600 \\
-3.57992052 & 3.88574346 & 0.19483491 \\
-3.38395864 & 8.14649885 & 0.74578973 \\
-5.86468074 & 8.23215048 & 0.89286330 \\
-7.19694955 & 6.15963514 & 0.67502881 \\
7.65945824 & 4.15000204 & 1.89034747 \\
5.12099940 & 2.76752158 & -1.29127804 \\
6.45276965 & 0.70771378 & -1.45423784 \\
9.01488899 & 2.11107318 & 1.69343602 \\
6.45252734 & -0.70764088 & 1.45394912 \\
5.12077307 & -2.76744071 & 1.29073989 \\
7.65965907 & -4.14982042 & -1.89059057 \\
9.01508732 & -2.11091358 & -1.69340192 \\
12.20583663 & 0.90112082 & 1.38238307 \\
13.55823487 & 2.93436554 & 0.94174455 \\
11.05776236 & 3.84718706 & -2.43656322 \\
\hline .70764529 & 1.85569196 & -1.97626728 \\
-12.20605069 & -0.90135448 & -1.38156149 \\
13.55817148 & -2.93471004 & -0.94060592 \\
-11.0565696948 & -3.84725314 & 2.43718856 \\
-13.70640244 & -6.28429300 & 1.93259631 \\
-13.03010688 & -4.99640527 & 2.96806020
\end{tabular}


$\mathrm{H}$

$\mathrm{H}$

$\mathrm{H}$

$\mathrm{H}$

$\mathrm{H}$

$\mathrm{H}$

$\mathrm{H}$

\section{$(P, P)$-TPEMC}

$\mathrm{C}$

C

C

C

C

C

C

C

C

C

C

C

C

C

C

C

C

C

C

C

C

C

C

C

C

C

C

C

C

C

C

C

C

C

C

$\begin{array}{rrr}-11.95124348 & -5.95475427 & 1.91062525 \\ 13.70602313 & -6.28503004 & 1.93091801 \\ 13.03010963 & -4.99718062 & 2.96672029 \\ 11.95093947 & -5.95521465 & 1.90931539 \\ 13.70700427 & 6.28471692 & -1.92967708 \\ 13.03120444 & 4.99694655 & -2.96565167 \\ 11.95188627 & 5.95506056 & -1.90846972\end{array}$

$$
\begin{array}{ccc}
-7.28207030 & -3.27401120 & -1.43728479 \\
-6.21589462 & -3.58224978 & -0.57508428 \\
-5.94523654 & -2.68542006 & 0.47209754 \\
-6.70902426 & -1.53635148 & 0.65083018 \\
-7.79454979 & -1.24284498 & -0.19307545 \\
-8.05392559 & -2.13231018 & -1.24998776 \\
-5.40669132 & -4.81256595 & -0.76337370 \\
-8.60004768 & -0.00000156 & -0.00006741 \\
-7.79454582 & 1.24281999 & 0.19304763 \\
-9.96905473 & 0.00002156 & -0.00014607 \\
-6.70892731 & 1.53633618 & -0.65073316 \\
-5.94515743 & 2.68540080 & -0.47189673 \\
-6.21592467 & 3.58221521 & 0.57527084 \\
-7.28220000 & 3.27396567 & 1.43734595 \\
-8.05404132 & 2.13227338 & 1.24994146 \\
-5.40673567 & 4.81252454 & 0.76366433 \\
-10.77267595 & 1.25408016 & -0.09007656 \\
-10.77266473 & -1.25407520 & 0.08971765 \\
-10.50157648 & -2.23750242 & 1.05119812 \\
-11.27156722 & -3.39690452 & 1.15630598 \\
-12.34572475 & -3.59693451 & 0.28140716 \\
-12.64183461 & -2.61965104 & -0.68100144 \\
-11.87472270 & -1.46706169 & -0.76292965 \\
-11.87505005 & 1.46686453 & 0.76221486 \\
-12.64220212 & 2.61942499 & 0.68022562 \\
-12.34581434 & 3.59691161 & -0.28188684 \\
-11.27132528 & 3.39708656 & -1.15644137 \\
-10.50130920 & 2.23771753 & -1.05127750 \\
-6.00244933 & -6.00727284 & -1.20638023 \\
-5.24514503 & -7.16595924 & -1.37609127 \\
-3.88064234 & -7.16570163 & -1.10497397 \\
-3.25760960 & -5.98257455 & -0.65890538 \\
-4.03100365 & -4.81741704 & -0.49744052 \\
-4.03103432 & 4.81739589 & 0.49779804 \\
-3.25765288 & 5.98254983 & 0.65934696
\end{array}
$$




\begin{tabular}{|c|c|c|c|}
\hline $\mathrm{C}$ & -3.88070817 & 7.16565226 & 1.10544840 \\
\hline $\mathrm{C}$ & -5.24522280 & 7.16588965 & 1.37650531 \\
\hline $\mathrm{C}$ & -6.00251580 & 6.00720955 & 1.20670074 \\
\hline $\mathrm{C}$ & -1.86292366 & -5.96471254 & -0.37944430 \\
\hline $\mathrm{C}$ & -1.86295903 & 5.96470603 & 0.37992354 \\
\hline $\mathrm{C}$ & -0.66614046 & -5.96027290 & -0.13568787 \\
\hline $\mathrm{C}$ & -0.66617271 & 5.96026692 & 0.13618203 \\
\hline $\mathrm{C}$ & 7.28208882 & 3.27397826 & -1.43730268 \\
\hline $\mathrm{C}$ & 6.21588779 & 3.58222695 & -0.57513519 \\
\hline $\mathrm{C}$ & 5.94520527 & 2.68540728 & 0.47204918 \\
\hline $\mathrm{C}$ & 6.70898748 & 1.53634005 & 0.65081432 \\
\hline $\mathrm{C}$ & 7.79453507 & 1.24282287 & -0.19305889 \\
\hline $\mathrm{C}$ & 8.05394014 & 2.13228157 & -1.24997180 \\
\hline $\mathrm{C}$ & 5.40668815 & 4.81253951 & -0.76345580 \\
\hline $\mathrm{C}$ & 8.60004154 & -0.00000521 & -0.00001869 \\
\hline $\mathrm{C}$ & 7.79455460 & -1.24283481 & 0.19309841 \\
\hline $\mathrm{C}$ & 9.96905346 & 0.00000285 & -0.00008516 \\
\hline $\mathrm{C}$ & 6.70895308 & -1.53637391 & -0.65069735 \\
\hline $\mathrm{C}$ & 5.94518336 & -2.68543742 & -0.47185543 \\
\hline $\mathrm{C}$ & 6.21592975 & -3.58222967 & 0.57533594 \\
\hline $\mathrm{C}$ & 7.28218228 & -3.27395538 & 1.43743059 \\
\hline $\mathrm{C}$ & 8.05402254 & -2.13226421 & 1.25002079 \\
\hline $\mathrm{C}$ & 5.40674059 & -4.81253659 & 0.76373746 \\
\hline $\mathrm{C}$ & 10.77266986 & -1.25406673 & -0.09007466 \\
\hline $\mathrm{C}$ & 10.77266049 & 1.25408114 & 0.08980990 \\
\hline $\mathrm{C}$ & 11.87484172 & 1.46700439 & -0.76269370 \\
\hline $\mathrm{C}$ & 12.64196897 & 2.61958328 & -0.68073167 \\
\hline $\mathrm{C}$ & 12.34575274 & 3.59694253 & 0.28156446 \\
\hline $\mathrm{C}$ & 11.27145884 & 3.39697594 & 1.15632520 \\
\hline $\mathrm{C}$ & 10.50146213 & 2.23758999 & 1.05118663 \\
\hline $\mathrm{C}$ & 11.87492458 & -1.46699426 & 0.76233224 \\
\hline $\mathrm{C}$ & 12.64205713 & -2.61956330 & 0.68028248 \\
\hline $\mathrm{C}$ & 12.34577158 & -3.59690484 & -0.28201062 \\
\hline $\mathrm{C}$ & 11.27140598 & -3.39693057 & -1.15668061 \\
\hline $\mathrm{C}$ & 10.50140593 & -2.23755399 & -1.05145407 \\
\hline $\mathrm{C}$ & 6.00244219 & 6.00722976 & -1.20651342 \\
\hline $\mathrm{C}$ & 5.24514048 & 7.16591388 & -1.37625095 \\
\hline $\mathrm{C}$ & 3.88064264 & 7.16567369 & -1.10510851 \\
\hline $\mathrm{C}$ & 3.25761330 & 5.98256449 & -0.65898907 \\
\hline $\mathrm{C}$ & 4.03100419 & 4.81740818 & -0.49750089 \\
\hline $\mathrm{C}$ & 4.03104499 & -4.81741656 & 0.49784171 \\
\hline $\mathrm{C}$ & 3.25766255 & -5.98256915 & 0.65939864 \\
\hline $\mathrm{C}$ & 3.88071171 & -7.16566221 & 1.10553280 \\
\hline $\mathrm{C}$ & 5.24522075 & -7.16589076 & 1.37661868 \\
\hline
\end{tabular}




\begin{tabular}{|c|c|c|c|}
\hline $\mathrm{C}$ & 6.00251437 & -6.00721188 & 1.20680911 \\
\hline $\mathrm{C}$ & 1.86293509 & 5.96471656 & -0.37948726 \\
\hline $\mathrm{C}$ & 1.86297484 & -5.96473254 & 0.37994364 \\
\hline $\mathrm{C}$ & 0.66619469 & -5.96029338 & 0.13617259 \\
\hline $\mathrm{C}$ & 0.66615961 & 5.96027534 & -0.13569323 \\
\hline $\mathrm{O}$ & -13.16261138 & 4.69024008 & -0.28769114 \\
\hline $\mathrm{C}$ & -12.91409598 & 5.70918205 & -1.24283860 \\
\hline $\mathrm{O}$ & -13.16245845 & -4.69032191 & 0.28711087 \\
\hline $\mathrm{C}$ & -12.91427405 & -5.70900547 & 1.24261990 \\
\hline $\mathrm{O}$ & 13.16253989 & -4.69025661 & -0.28785301 \\
\hline $\mathrm{C}$ & 12.91420875 & -5.70899769 & -1.24326163 \\
\hline $\mathrm{O}$ & 13.16251184 & 4.69030099 & 0.28732097 \\
\hline $\mathrm{C}$ & 12.91420833 & 5.70908871 & 1.2426874 \\
\hline $\mathrm{H}$ & -7.49122121 & -3.92256715 & -2.28356873 \\
\hline $\mathrm{H}$ & -5.14215616 & -2.90344242 & 1.17067917 \\
\hline $\mathrm{H}$ & -6.47435331 & -0.86153679 & 1.46921342 \\
\hline $\mathrm{H}$ & -8.86737733 & -1.91778380 & -1.93592650 \\
\hline $\mathrm{H}$ & -6.47416741 & 0.86153414 & -1.46910150 \\
\hline $\mathrm{H}$ & -5.14200460 & 2.90342986 & -1.17039233 \\
\hline $\mathrm{H}$ & -7.49144346 & 3.92250552 & 2.28361924 \\
\hline $\mathrm{H}$ & -8.86757805 & 1.91774064 & 1.93577886 \\
\hline $\mathrm{H}$ & -9.67225995 & -2.09463466 & 1.73682806 \\
\hline $\mathrm{H}$ & -11.02884975 & -4.12652789 & 1.92060039 \\
\hline $\mathrm{H}$ & -13.48185449 & -2.78641973 & -1.34826405 \\
\hline $\mathrm{H}$ & -12.12444023 & -0.71511481 & -1.50596738 \\
\hline $\mathrm{H}$ & -12.12499708 & 0.71478097 & 1.50503517 \\
\hline $\mathrm{H}$ & -13.48246590 & 2.78600465 & 1.34722865 \\
\hline $\mathrm{H}$ & -11.02837968 & 4.12685505 & -1.92052396 \\
\hline $\mathrm{H}$ & -9.67175956 & 2.09502221 & -1.73666001 \\
\hline $\mathrm{H}$ & -7.07202673 & -6.03267615 & -1.39297768 \\
\hline $\mathrm{H}$ & -5.72706274 & -8.07978077 & -1.71300699 \\
\hline $\mathrm{H}$ & -3.28727865 & -8.06544848 & -1.23196519 \\
\hline $\mathrm{H}$ & -3.53729036 & -3.90471596 & -0.18023585 \\
\hline $\mathrm{H}$ & -3.53729761 & 3.90471330 & 0.18057806 \\
\hline $\mathrm{H}$ & -3.28735446 & 8.06539598 & 1.23250806 \\
\hline $\mathrm{H}$ & -5.72716000 & 8.07969176 & 1.71344583 \\
\hline $\mathrm{H}$ & -7.07210208 & 6.03260662 & 1.39324722 \\
\hline $\mathrm{H}$ & 7.49126259 & 3.92252271 & -2.28358986 \\
\hline $\mathrm{H}$ & 5.14210996 & 2.90343464 & 1.17061182 \\
\hline $\mathrm{H}$ & 6.47429525 & 0.86153652 & 1.46920064 \\
\hline $\mathrm{H}$ & 8.86741200 & 1.91774789 & -1.93588492 \\
\hline $\mathrm{H}$ & 6.47420842 & -0.86159163 & -1.46908629 \\
\hline $\mathrm{H}$ & 5.14204693 & -2.90348282 & -1.17036504 \\
\hline $\mathrm{H}$ & 7.49140748 & -3.92247454 & 2.28372435 \\
\hline
\end{tabular}




$\begin{array}{lrrr}\mathrm{H} & 8.86753466 & -1.91771235 & 1.93588034 \\ \mathrm{H} & 12.12465418 & 0.71501723 & -1.50565767 \\ \mathrm{H} & 13.48208309 & 2.78627667 & -1.34789479 \\ \mathrm{H} & 11.02864882 & 4.12664688 & 1.92054400 \\ \mathrm{H} & 9.67205971 & 2.09478048 & 1.73672408 \\ \mathrm{H} & 12.12478975 & -0.71501905 & 1.50529059 \\ \mathrm{H} & 13.48222819 & -2.78626337 & 1.34737215 \\ \mathrm{H} & 11.02854145 & -4.12658851 & -1.92089438 \\ \mathrm{H} & 9.67194641 & -2.09473737 & -1.73692072 \\ \mathrm{H} & 7.07201651 & 6.03262582 & -1.39312944 \\ \mathrm{H} & 5.72705766 & 8.07972081 & -1.71320713 \\ \mathrm{H} & 3.28728209 & 8.06542011 & -1.23211703 \\ \mathrm{H} & 3.53728824 & 3.90472152 & -0.18025964 \\ \mathrm{H} & 3.53731353 & -3.90474164 & 0.18059105 \\ \mathrm{H} & 3.28735799 & -8.06540540 & 1.23259578 \\ \mathrm{H} & 5.72715349 & -8.07968557 & 1.71358547 \\ \mathrm{H} & 7.07209690 & -6.03260275 & 1.39337803 \\ \mathrm{H} & -13.67066551 & 6.47602122 & -1.06636056 \\ \mathrm{H} & -11.91582972 & 6.14840949 & -1.11502748 \\ \mathrm{H} & -13.01243035 & 5.33315717 & -2.26986695 \\ \mathrm{H} & -13.67075886 & -6.47590718 & 1.06605759 \\ \mathrm{H} & -13.01300360 & -5.33270816 & 2.26951614 \\ \mathrm{H} & -11.91595731 & -6.14825857 & 1.11530781 \\ \mathrm{H} & 13.67077997 & -6.47584765 & -1.06683704 \\ \mathrm{H} & 11.91594016 & -6.14829349 & -1.11569962 \\ \mathrm{H} & 13.01268425 & -5.33274203 & -2.27019186 \\ \mathrm{H} & 13.67073621 & 6.47595841 & 1.06616309 \\ \mathrm{H} & 11.91591270 & 6.14833583 & 1.11517122 \\ \mathrm{H} & 13.01277357 & 5.33289813 & 2.26963297 \\ & & & \\ & & & \\ & & & \end{array}$

Optimized Cartesian coordinates of TPEMCSat B3LYP/6-31G* level of theory (M,M)-(in,in)-TPEMCS

$\begin{array}{cccc}\text { C } & 6.00544945 & -2.97312024 & -1.96545438 \\ \mathrm{C} & 5.06329031 & -3.53231309 & -1.08374654 \\ \mathrm{C} & 4.84831605 & -2.86669370 & 0.13601263 \\ \mathrm{C} & 5.55667963 & -1.71509104 & 0.46585298 \\ \mathrm{C} & 6.54590204 & -1.19971716 & -0.38827840 \\ \mathrm{C} & 6.73748627 & -1.84190424 & -1.62196035 \\ \mathrm{C} & 7.34639317 & -0.00023356 & -0.00034438 \\ \mathrm{C} & 6.54614340 & 1.19913359 & 0.38848039 \\ \mathrm{C} & 8.71323652 & -0.00012446 & -0.00109325 \\ \mathrm{C} & 9.51774094 & 1.24791439 & 0.15687030 \\ \mathrm{C} & 9.51773829 & -1.24803864 & -0.16001906\end{array}$




\begin{tabular}{|c|c|c|c|}
\hline $\mathrm{C}$ & 5.55625438 & 1.71466922 & -0.46477613 \\
\hline $\mathrm{C}$ & 4.84811619 & 2.86617969 & -0.13414011 \\
\hline $\mathrm{C}$ & 5.06395206 & 3.53151110 & 1.08562846 \\
\hline $\mathrm{C}$ & 6.00677975 & 2.97211956 & 1.96649635 \\
\hline $\mathrm{C}$ & 6.73862363 & 1.84102864 & 1.62217019 \\
\hline $\mathrm{C}$ & 10.60850013 & 1.29161169 & 1.04829636 \\
\hline $\mathrm{C}$ & 11.37737679 & 2.43643792 & 1.19692292 \\
\hline $\mathrm{C}$ & 11.09436883 & 3.57989940 & 0.43449053 \\
\hline $\mathrm{C}$ & 10.03211329 & 3.55268990 & -0.47631380 \\
\hline $\mathrm{C}$ & 9.26043025 & 2.39649345 & -0.60372773 \\
\hline $\mathrm{C}$ & 9.26140541 & -2.39671910 & 0.60075521 \\
\hline $\mathrm{C}$ & 10.03308486 & -3.55281251 & 0.47236171 \\
\hline $\mathrm{C}$ & 11.09432311 & -3.57980687 & -0.43963488 \\
\hline $\mathrm{C}$ & 11.37635077 & -2.43623622 & -1.20226663 \\
\hline $\mathrm{C}$ & 10.60750454 & -1.29151723 & -1.05266698 \\
\hline $\mathrm{C}$ & 4.35374161 & -4.79399066 & -1.41996094 \\
\hline $\mathrm{C}$ & 4.35456933 & 4.79306328 & 1.42269548 \\
\hline $\mathrm{C}$ & 4.95824594 & -5.74947260 & -2.25606718 \\
\hline $\mathrm{C}$ & 4.30692644 & -6.94283535 & -2.55952508 \\
\hline $\mathrm{C}$ & 3.05368144 & -7.22073035 & -2.02469340 \\
\hline $\mathrm{C}$ & 2.43180488 & -6.30054610 & -1.16004970 \\
\hline $\mathrm{C}$ & 3.08900863 & -5.08996690 & -0.88678202 \\
\hline $\mathrm{C}$ & 3.08967640 & 5.08935532 & 0.89006721 \\
\hline $\mathrm{C}$ & 2.43259906 & 6.29982922 & 1.16410254 \\
\hline $\mathrm{C}$ & 3.05475505 & 7.21955478 & 2.02903251 \\
\hline $\mathrm{C}$ & 4.30815592 & 6.94135038 & 2.56333485 \\
\hline $\mathrm{C}$ & 4.95936211 & 5.74813172 & 2.25906772 \\
\hline $\mathrm{C}$ & -6.00544802 & 2.97311654 & -1.96545764 \\
\hline $\mathrm{C}$ & -5.06328956 & 3.53231071 & -1.08374990 \\
\hline $\mathrm{C}$ & -4.84831625 & 2.86669313 & 0.13601043 \\
\hline $\mathrm{C}$ & -5.55668010 & 1.71509097 & 0.46585194 \\
\hline $\mathrm{C}$ & -6.54590193 & 1.19971588 & -0.38827939 \\
\hline $\mathrm{C}$ & -6.73748516 & 1.84190109 & -1.62196249 \\
\hline $\mathrm{C}$ & -7.34639333 & 0.00023285 & -0.00034416 \\
\hline $\mathrm{C}$ & -6.54614334 & -1.19913379 & 0.38848187 \\
\hline $\mathrm{C}$ & -8.71323673 & 0.00012422 & -0.00109279 \\
\hline $\mathrm{C}$ & -9.51774205 & -1.24791414 & 0.15687037 \\
\hline $\mathrm{C}$ & -9.51773797 & 1.24803882 & -0.16001815 \\
\hline $\mathrm{C}$ & -6.73862183 & -1.84102631 & 1.62217327 \\
\hline $\mathrm{C}$ & -6.00677758 & -2.97211662 & 1.96650063 \\
\hline $\mathrm{C}$ & -5.06395114 & -3.53150998 & 1.08563253 \\
\hline $\mathrm{C}$ & -4.84811680 & -2.86618091 & -0.13413758 \\
\hline $\mathrm{C}$ & -5.55625533 & -1.71467102 & -0.46477487 \\
\hline $\mathrm{C}$ & -10.60849907 & -1.29161185 & 1.04829915 \\
\hline
\end{tabular}




\begin{tabular}{|c|c|c|c|}
\hline $\mathrm{C}$ & -11.37737679 & -2.43643741 & 1.19692547 \\
\hline $\mathrm{C}$ & -11.09437237 & -3.57989768 & 0.43448997 \\
\hline $\mathrm{C}$ & -10.03211937 & -3.55268764 & -0.47631724 \\
\hline $\mathrm{C}$ & -9.26043511 & -2.39649195 & -0.60373087 \\
\hline $\mathrm{C}$ & -9.26140600 & 2.39671834 & 0.60075786 \\
\hline $\mathrm{C}$ & -10.03308502 & 3.55281203 & 0.47236466 \\
\hline $\mathrm{C}$ & -11.09432205 & 3.57980769 & -0.43963341 \\
\hline $\mathrm{C}$ & -11.37634881 & 2.43623800 & -1.20226690 \\
\hline $\mathrm{C}$ & -10.60750300 & 1.29151868 & -1.05266751 \\
\hline $\mathrm{C}$ & -4.35374058 & 4.79398776 & -1.41996569 \\
\hline $\mathrm{C}$ & -4.35456799 & -4.79306152 & 1.42270110 \\
\hline $\mathrm{C}$ & -4.95824420 & 5.74946832 & -2.25607402 \\
\hline $\mathrm{C}$ & -4.30692444 & 6.94283059 & -2.55953336 \\
\hline $\mathrm{C}$ & -3.05367988 & 7.22072643 & -2.02470112 \\
\hline $\mathrm{C}$ & -2.43180405 & 6.30054361 & -1.16005537 \\
\hline $\mathrm{C}$ & -3.08900803 & 5.08996487 & -0.88678621 \\
\hline $\mathrm{C}$ & -3.08967564 & -5.08935451 & 0.89007194 \\
\hline $\mathrm{C}$ & -2.43259794 & -6.29982784 & 1.16410881 \\
\hline $\mathrm{C}$ & -3.05475291 & -7.21955182 & 2.02904119 \\
\hline $\mathrm{C}$ & -4.30815319 & -6.94134651 & 2.56334442 \\
\hline $\mathrm{C}$ & -4.95935977 & -5.74812844 & 2.25907582 \\
\hline $\mathrm{C}$ & 0.63830564 & 7.90057934 & 0.31097573 \\
\hline $\mathrm{C}$ & -0.63770304 & 7.90076905 & -0.30555013 \\
\hline $\mathrm{C}$ & -1.14003415 & 6.64207566 & -0.55128512 \\
\hline $\mathrm{S}$ & 0.00037247 & 5.42837827 & 0.00180486 \\
\hline $\mathrm{C}$ & 1.14072397 & 6.64173413 & 0.55576591 \\
\hline $\mathrm{C}$ & 1.14003448 & -6.64207715 & -0.55127996 \\
\hline $\mathrm{S}$ & -0.00037274 & -5.42837886 & 0.00180683 \\
\hline $\mathrm{C}$ & -1.14072351 & -6.64173382 & 0.55577135 \\
\hline $\mathrm{C}$ & -0.63830569 & -7.90057944 & 0.31098221 \\
\hline $\mathrm{C}$ & 0.63770290 & -7.90077016 & -0.30554383 \\
\hline $\mathrm{O}$ & 11.91084085 & -4.65264545 & -0.65373416 \\
\hline $\mathrm{C}$ & 11.67305892 & -5.83570606 & 0.09182623 \\
\hline $\mathrm{O}$ & 11.91100241 & 4.65285375 & 0.64755895 \\
\hline $\mathrm{C}$ & 11.67222853 & 5.83581311 & -0.09784695 \\
\hline $\mathrm{O}$ & -11.91083926 & 4.65264668 & -0.65373256 \\
\hline $\mathrm{C}$ & -11.67305823 & 5.83570632 & 0.09182959 \\
\hline $\mathrm{O}$ & -11.91100685 & -4.65285138 & 0.64755835 \\
\hline $\mathrm{C}$ & -11.67223663 & -5.83580946 & -0.09785076 \\
\hline $\mathrm{H}$ & 6.16200908 & -3.42216566 & -2.94174260 \\
\hline $\mathrm{H}$ & 4.14287463 & -3.27238896 & 0.85533455 \\
\hline $\mathrm{H}$ & 5.36866491 & -1.22668763 & 1.41837088 \\
\hline $\mathrm{H}$ & 7.47298248 & -1.44732838 & -2.31654088 \\
\hline $\mathrm{H}$ & 5.36753194 & 1.22648347 & -1.41726646 \\
\hline
\end{tabular}


$\mathrm{H}$

$\mathrm{H}$

$\mathrm{H}$

$\mathrm{H}$

$\mathrm{H}$

$\mathrm{H}$

$\mathrm{H}$

$\mathrm{H}$

$\mathrm{H}$

$\mathrm{H}$

$\mathrm{H}$

$\mathrm{H}$

$\mathrm{H}$

$\mathrm{H}$

$\mathrm{H}$

$\mathrm{H}$

$\mathrm{H}$

$\mathrm{H}$

$\mathrm{H}$

$\mathrm{H}$

$\mathrm{H}$

$\mathrm{H}$

$\mathrm{H}$

$\mathrm{H}$

$\mathrm{H}$

$\mathrm{H}$

$\mathrm{H}$

$\mathrm{H}$

$\mathrm{H}$

$\mathrm{H}$

$\mathrm{H}$

$\mathrm{H}$

$\mathrm{H}$

$\mathrm{H}$

$\mathrm{H}$

$\mathrm{H}$

$\mathrm{H}$

$\mathrm{H}$

$\mathrm{H}$

$\mathrm{H}$

$\mathrm{H}$

$\mathrm{H}$

$\mathrm{H}$

$\mathrm{H}$
4.14214567

6.16404609

7.47466346

10.84836523

12.20868456

9.79861737

8.44053159

8.44228872

9.80038228

12.20688324

10.84660021

5.95366115

4.78435128

2.54611887

2.60590283

2.60633511

2.54731390

4.78580670

5.95490403

$-6.16200686$

$-4.14287537$

$-5.36866607$

$-7.47298073$

$-7.47466055$

$-6.16404255$

$-4.14214723$

$-5.36753406$

$-10.84836142$

$-12.20868271$

$-9.79862632$

$-8.44053863$

$-8.44229041$

$-9.80038324$

$-12.20688028$

$-10.84659804$

$-5.95365908$

$-4.78434873$

$-2.54611707$

$-2.60590276$

$-2.60633512$

$-2.54731140$

$-4.78580319$

$-5.95490128$

1.18375845
3.27201635

3.42089764

1.44632017

0.41085207

2.47036155

4.41497513

2.39032872

$-2.39072263$

$-4.41518851$

$-2.46999240$

$-0.41066737$

$-5.56978883$

$-7.66548600$

$-8.14431827$

$-4.36687644$

4.36660687

8.14303698

7.66365890

5.56826443

3.42216048

3.27238943

1.22668895

1.44732416

$-1.44631637$

$-3.42089272$

$-3.27201897$

$-1.22648707$

$-0.41085314$

$-2.47036138$

$-4.41497185$

$-2.39032679$

2.39072085

4.41518723

2.46999517

0.41066958

5.56978385

7.66548012

8.14431388

4.36687550

$-4.36660728$

$-8.14303348$

$-7.66365382$

$-5.56826046$

8.80722422
$-0.85286000$

2.94279163

2.31610106

1.63714964

1.89443863

$-1.09052412$

$-1.31485489$

1.31278784

1.08674412

$-1.90071318$

$-1.64169771$

$-2.64918151$

$-3.21614715$

$-2.28440011$

$-0.23638607$

0.23947079

2.28935012

3.22016910

2.65177107

$-2.94174667$

0.85533229

1.41837070

$-2.31654302$

2.31610444

2.94279705

$-0.85285759$

$-1.41726636$

1.63715486

1.89444334

$-1.09053010$

$-1.31486046$

1.31279171

1.08674846

$-1.90071458$

$-1.64169965$

$-2.64918890$

$-3.21615703$

$-2.28440893$

$-0.23638868$

0.23947361

2.28935996

3.22018056

2.65177996

0.54848473 


$\begin{array}{lrrr}\mathrm{H} & -1.18320977 & 8.80755162 & -0.54241064 \\ \mathrm{H} & -1.18375847 & -8.80722390 & 0.54849283 \\ \mathrm{H} & 1.18320962 & -8.80755316 & -0.54240286 \\ \mathrm{H} & 12.42663891 & -6.55371433 & -0.23717440 \\ \mathrm{H} & 11.78407903 & -5.66307690 & 1.17065158 \\ \mathrm{H} & 10.67278522 & -6.24360922 & -0.10529775 \\ \mathrm{H} & 12.42609259 & 6.55394585 & 0.23022984 \\ \mathrm{H} & 11.78204821 & 5.66309341 & -1.17678067 \\ \mathrm{H} & 10.67212878 & 6.24361112 & 0.10037176 \\ \mathrm{H} & -12.42663764 & 6.55371518 & -0.23717123 \\ \mathrm{H} & -11.78407983 & 5.66307595 & 1.17065458 \\ \mathrm{H} & -10.67278416 & 6.24360964 & -0.10529250 \\ \mathrm{H} & -12.42610079 & -6.55394188 & 0.23022651 \\ \mathrm{H} & -11.78205900 & -5.66308728 & -1.17678381 \\ \mathrm{H} & -10.67213692 & -6.24360932 & 0.10036437\end{array}$

\section{(M,P)-(in,in)-TPEMCS}

\begin{tabular}{|c|c|c|c|}
\hline $\mathrm{C}$ & -6.25462325 & 3.39746688 & -1.27540925 \\
\hline $\mathrm{C}$ & -5.22132472 & 3.74173825 & -0.38651721 \\
\hline $\mathrm{C}$ & -4.84146959 & 2.78715189 & 0.57128958 \\
\hline $\mathrm{C}$ & -5.50767178 & 1.56710639 & 0.67452406 \\
\hline $\mathrm{C}$ & -6.59456195 & 1.25868388 & -0.16200448 \\
\hline $\mathrm{C}$ & -6.92280425 & 2.18520555 & -1.16735422 \\
\hline $\mathrm{C}$ & -7.37830367 & -0.00006365 & 0.00006229 \\
\hline $\mathrm{C}$ & -6.59455655 & -1.25881672 & 0.16207260 \\
\hline $\mathrm{C}$ & -8.75016050 & -0.00003546 & 0.00010247 \\
\hline $\mathrm{C}$ & -9.55772631 & -1.24538621 & -0.15834078 \\
\hline $\mathrm{C}$ & -9.55763053 & 1.24537467 & 0.15855379 \\
\hline $\mathrm{C}$ & -5.50773406 & -1.56723913 & -0.67454469 \\
\hline $\mathrm{C}$ & -4.84150777 & -2.78727506 & -0.57135204 \\
\hline $\mathrm{C}$ & -5.22126814 & -3.74185202 & 0.38650064 \\
\hline $\mathrm{C}$ & -6.25448873 & -3.39757784 & 1.27548252 \\
\hline $\mathrm{C}$ & -6.92269005 & -2.18532410 & 1.16747269 \\
\hline $\mathrm{C}$ & -10.66254125 & -1.50361759 & 0.67794033 \\
\hline $\mathrm{C}$ & -11.43604609 & -2.64466163 & 0.52506209 \\
\hline $\mathrm{C}$ & -11.14433454 & -3.56310719 & -0.49481346 \\
\hline $\mathrm{C}$ & -10.06760484 & -3.31643260 & -1.35457473 \\
\hline $\mathrm{C}$ & -9.29128895 & -2.17005792 & -1.17789812 \\
\hline $\mathrm{C}$ & -9.29103018 & 2.17008222 & 1.17803563 \\
\hline $\mathrm{C}$ & -10.06724552 & 3.31652351 & 1.35472178 \\
\hline $\mathrm{C}$ & -11.14403433 & 3.56323207 & 0.49504433 \\
\hline $\mathrm{C}$ & -11.43590342 & 2.64475580 & -0.52475877 \\
\hline $\mathrm{C}$ & -10.66249547 & 1.50364759 & -0.67764820 \\
\hline $\mathrm{C}$ & -4.61284452 & 5.09542442 & -0.43410928 \\
\hline
\end{tabular}




\begin{tabular}{|c|c|c|c|}
\hline $\mathrm{C}$ & -4.61276632 & -5.09552979 & 0.43405782 \\
\hline $\mathrm{C}$ & -5.43565208 & 6.21021231 & -0.67325709 \\
\hline $\mathrm{C}$ & -4.90241592 & 7.49644699 & -0.69028154 \\
\hline $\mathrm{C}$ & -3.54333380 & 7.69583775 & -0.47279862 \\
\hline $\mathrm{C}$ & -2.69337566 & 6.60210410 & -0.22311921 \\
\hline $\mathrm{C}$ & -3.24402668 & 5.30970710 & -0.21053515 \\
\hline $\mathrm{C}$ & -3.24395675 & -5.30979103 & 0.21041321 \\
\hline $\mathrm{C}$ & -2.69328634 & -6.60218014 & 0.22296285 \\
\hline $\mathrm{C}$ & -3.54321726 & -7.69592782 & 0.47267340 \\
\hline $\mathrm{C}$ & -4.90229108 & -7.49655785 & 0.69022804 \\
\hline $\mathrm{C}$ & -5.43554587 & -6.21033032 & 0.67324272 \\
\hline $\mathrm{C}$ & 6.25459107 & -3.39754725 & 1.27531857 \\
\hline $\mathrm{C}$ & 5.22133176 & -3.74180121 & 0.38637420 \\
\hline $\mathrm{C}$ & 4.84151614 & -2.78719621 & -0.57142896 \\
\hline $\mathrm{C}$ & 5.50772031 & -1.56714710 & -0.67460804 \\
\hline $\mathrm{C}$ & 6.59457142 & -1.25873753 & 0.16197682 \\
\hline $\mathrm{C}$ & 6.92277107 & -2.18528035 & 1.16732187 \\
\hline $\mathrm{C}$ & 7.37830351 & 0.00002392 & -0.00002268 \\
\hline $\mathrm{C}$ & 6.59454973 & 1.25876621 & -0.16208647 \\
\hline $\mathrm{C}$ & 8.75016008 & 0.00003812 & 0.00003949 \\
\hline $\mathrm{C}$ & 9.55766254 & 1.24542239 & 0.15853864 \\
\hline $\mathrm{C}$ & 9.55769080 & -1.24533933 & -0.15835915 \\
\hline $\mathrm{C}$ & 6.92274561 & 2.18527255 & -1.16746624 \\
\hline $\mathrm{C}$ & 6.25454652 & 3.39752359 & -1.27552224 \\
\hline $\mathrm{C}$ & 5.22127149 & 3.74179861 & -0.38660404 \\
\hline $\mathrm{C}$ & 4.84145476 & 2.78722269 & 0.57122800 \\
\hline $\mathrm{C}$ & 5.50767570 & 1.56718750 & 0.67446359 \\
\hline $\mathrm{C}$ & 10.66254961 & 1.50368646 & -0.67763683 \\
\hline $\mathrm{C}$ & 11.43599026 & 2.64476644 & -0.52470074 \\
\hline $\mathrm{C}$ & 11.14413567 & 3.56321898 & 0.49512771 \\
\hline $\mathrm{C}$ & 10.06732903 & 3.31651445 & 1.35478402 \\
\hline $\mathrm{C}$ & 9.29108099 & 2.17010289 & 1.17805030 \\
\hline $\mathrm{C}$ & 9.29121037 & -2.17004820 & -1.17787103 \\
\hline $\mathrm{C}$ & 10.06748889 & -3.31645478 & -1.35450657 \\
\hline $\mathrm{C}$ & 11.14422045 & -3.56312520 & -0.49474637 \\
\hline $\mathrm{C}$ & 11.43596957 & -2.64464643 & 0.52508880 \\
\hline $\mathrm{C}$ & 10.66250119 & -1.50357253 & 0.67792724 \\
\hline $\mathrm{C}$ & 4.61284867 & -5.09548785 & 0.43391493 \\
\hline $\mathrm{C}$ & 4.61277358 & 5.09547675 & -0.43419922 \\
\hline $\mathrm{C}$ & 5.43565118 & -6.21028370 & 0.67304189 \\
\hline $\mathrm{C}$ & 4.90241216 & -7.49651790 & 0.69002113 \\
\hline $\mathrm{C}$ & 3.54333173 & -7.69589882 & 0.47251790 \\
\hline $\mathrm{C}$ & 2.69337826 & -6.60215596 & 0.22286290 \\
\hline $\mathrm{C}$ & 3.24403300 & -5.30976012 & 0.21031849 \\
\hline
\end{tabular}




\begin{tabular}{|c|c|c|c|}
\hline $\mathrm{C}$ & 3.24395825 & 5.30974483 & -0.21059592 \\
\hline $\mathrm{C}$ & 2.69329120 & 6.60213501 & -0.22317735 \\
\hline $\mathrm{C}$ & 3.54323067 & 7.69587707 & -0.47288393 \\
\hline $\mathrm{C}$ & 4.90231005 & 7.49650075 & -0.69039733 \\
\hline $\mathrm{C}$ & 5.43556217 & 6.21027269 & -0.67337532 \\
\hline $\mathrm{C}$ & -0.70880498 & -7.96525374 & -0.61085167 \\
\hline $\mathrm{C}$ & 0.70887843 & -7.96524607 & -0.61088021 \\
\hline $\mathrm{C}$ & 1.26546037 & -6.86035128 & -0.00703981 \\
\hline S & 0.00004873 & -5.79155019 & 0.57054239 \\
\hline $\mathrm{C}$ & -1.26537462 & -6.86036318 & -0.00699211 \\
\hline $\mathrm{C}$ & -1.26545870 & 6.86031000 & 0.00677631 \\
\hline $\mathrm{S}$ & -0.00004124 & 5.79149203 & -0.57076202 \\
\hline $\mathrm{C}$ & 1.26537630 & 6.86032498 & 0.00674866 \\
\hline $\mathrm{C}$ & 0.70880034 & 7.96523088 & 0.61057444 \\
\hline $\mathrm{C}$ & -0.70888277 & 7.96522188 & 0.61059099 \\
\hline $\mathrm{O}$ & -11.96749912 & 4.64956236 & 0.56850664 \\
\hline $\mathrm{C}$ & -11.72638331 & 5.60687770 & 1.58690118 \\
\hline $\mathrm{O}$ & -11.96788753 & -4.64937183 & -0.56825956 \\
\hline $\mathrm{C}$ & -11.72693731 & -5.60664994 & -1.58672802 \\
\hline $\mathrm{O}$ & 11.96773846 & -4.64941884 & -0.56815414 \\
\hline $\mathrm{C}$ & 11.72674834 & -5.60673233 & -1.58657991 \\
\hline $\mathrm{O}$ & 11.96763326 & 4.64952132 & 0.56863667 \\
\hline $\mathrm{C}$ & 11.72653418 & 5.60681033 & 1.58705992 \\
\hline $\mathrm{H}$ & -6.53162801 & 4.08955469 & -2.06571857 \\
\hline $\mathrm{H}$ & -4.04801267 & 3.02182639 & 1.27600106 \\
\hline $\mathrm{H}$ & -5.20996848 & 0.85902867 & 1.44310790 \\
\hline $\mathrm{H}$ & -7.72512173 & 1.95256801 & -1.86012283 \\
\hline $\mathrm{H}$ & -5.21010520 & -0.85916856 & -1.44316369 \\
\hline $\mathrm{H}$ & -4.04810893 & -3.02195051 & -1.27612863 \\
\hline $\mathrm{H}$ & -6.53141142 & -4.08965608 & 2.06582902 \\
\hline $\mathrm{H}$ & -7.72493700 & -1.95268115 & 1.86031994 \\
\hline $\mathrm{H}$ & -10.90934206 & -0.79669658 & 1.46482729 \\
\hline $\mathrm{H}$ & -12.27812611 & -2.84668938 & 1.17991679 \\
\hline $\mathrm{H}$ & -9.82809863 & -3.99950321 & -2.16167231 \\
\hline $\mathrm{H}$ & -8.45924293 & -1.99074578 & -1.85167433 \\
\hline $\mathrm{H}$ & -8.45893521 & 1.99074567 & 1.85174518 \\
\hline $\mathrm{H}$ & -9.82761533 & 3.99961997 & 2.16176043 \\
\hline $\mathrm{H}$ & -12.27802509 & 2.84681271 & -1.17955090 \\
\hline $\mathrm{H}$ & -10.90941577 & 0.79670564 & -1.46447897 \\
\hline $\mathrm{H}$ & -6.50180284 & 6.06447552 & -0.81792144 \\
\hline $\mathrm{H}$ & -5.55011010 & 8.34882723 & -0.87810674 \\
\hline $\mathrm{H}$ & -3.12498051 & 8.69632867 & -0.52041765 \\
\hline $\mathrm{H}$ & -2.59589850 & 4.45748513 & -0.02346725 \\
\hline $\mathrm{H}$ & -2.59585113 & -4.45755805 & 0.02331690 \\
\hline
\end{tabular}


H

$\mathrm{H}$

$\mathrm{H}$

$\mathrm{H}$

$\mathrm{H}$

$\mathrm{H}$

$\mathrm{H}$

$\mathrm{H}$

$\mathrm{H}$

$\mathrm{H}$

$\mathrm{H}$

$\mathrm{H}$

$\mathrm{H}$

$\mathrm{H}$

$\mathrm{H}$

$\mathrm{H}$

$\mathrm{H}$

$\mathrm{H}$

$\mathrm{H}$

$\mathrm{H}$

$\mathrm{H}$

$\mathrm{H}$

$\mathrm{H}$

$\mathrm{H}$

$\mathrm{H}$

$\mathrm{H}$

$\mathrm{H}$

$\mathrm{H}$

$\mathrm{H}$

$\mathrm{H}$

$\mathrm{H}$

$\mathrm{H}$

$\mathrm{H}$

$\mathrm{H}$

$\mathrm{H}$

$\mathrm{H}$

$\mathrm{H}$

$\mathrm{H}$

$\mathrm{H}$

$\mathrm{H}$

$\mathrm{H}$

$\mathrm{H}$

$\mathrm{H}$

\begin{tabular}{rcc}
-3.12484723 & -8.69641308 & 0.52026409 \\
-5.54996352 & -8.34894889 & 0.87807943 \\
-6.50169087 & -6.06460813 & 0.81796467 \\
6.53156239 & -4.08965263 & 2.06562435 \\
4.04808884 & -3.02185789 & -1.27617808 \\
5.21004854 & -0.85905370 & -1.44318943 \\
7.72505246 & -1.95265327 & 1.86013526 \\
7.72504082 & 1.95263164 & -1.86025897 \\
6.53151725 & 4.08959982 & -2.06585356 \\
4.04801440 & 3.02189835 & 1.27595776 \\
5.21000176 & 0.85911828 & 1.44306660 \\
10.90945990 & 0.79676174 & -1.46448614 \\
12.27812749 & 2.84681813 & -1.17947453 \\
9.82771053 & 3.99959084 & 2.16184314 \\
8.45897394 & 1.99076865 & 1.85174543 \\
8.45916037 & -1.99073991 & -1.85164378 \\
9.82795221 & -3.99955355 & -2.16157124 \\
12.27804858 & -2.84667365 & 1.17994495 \\
10.90932973 & -0.79662684 & 1.46478343 \\
6.50179988 & -6.06455156 & 0.81772705 \\
5.55010195 & -8.34890529 & 0.87782907 \\
3.12497514 & -8.69638990 & 0.52010458 \\
2.59590969 & -4.45753053 & 0.02326803 \\
2.59584517 & 4.45751650 & -0.02350457 \\
3.12486450 & 8.69636265 & -0.52050112 \\
5.54998966 & 8.34888746 & -0.87824357 \\
6.50171150 & 6.06454786 & -0.81806156 \\
-1.30837579 & -8.74665645 & -1.06524983 \\
1.30843948 & -8.74664279 & -1.06530160 \\
1.30836615 & 8.74664597 & 1.06495773 \\
-1.30844812 & 8.74662967 & 1.06498748 \\
-12.48892889 & 6.37768522 & 1.46022608 \\
-11.82102243 & 5.16482435 & 2.58769380 \\
-10.73154681 & 6.06118753 & 1.48772267 \\
-12.48952993 & -6.37740645 & -1.46002532 \\
-11.82163416 & -5.16453515 & -2.58748800 \\
-10.73212647 & -6.06104111 & -1.48766489 \\
12.48931837 & -6.37750710 & -1.45985252 \\
10.73192443 & -6.06108852 & -1.48748767 \\
11.82144879 & -5.16466241 & -2.58735936 \\
12.48910500 & 6.37759864 & 1.46041984 \\
10.73171281 & 6.06115398 & 1.48788405 \\
11.82114721 & 5.16472099 & 2.58783910 \\
& & \\
\hline
\end{tabular}




\section{(M,M)-(out,out)-TPEMCS}

\begin{tabular}{|c|c|c|c|}
\hline $\mathrm{C}$ & -5.49779141 & 2.46459801 & 0.59510590 \\
\hline $\mathrm{C}$ & -5.85803538 & 3.54283122 & -0.23006982 \\
\hline $\mathrm{C}$ & -7.08689915 & 3.46449928 & -0.90619570 \\
\hline $\mathrm{C}$ & -7.91046590 & 2.34923155 & -0.77764717 \\
\hline $\mathrm{C}$ & -7.54674984 & 1.26433444 & 0.03935702 \\
\hline $\mathrm{C}$ & -6.31952493 & 1.35013212 & 0.72123111 \\
\hline $\mathrm{C}$ & -8.37290226 & 0.02147741 & 0.12462861 \\
\hline $\mathrm{C}$ & -7.58170835 & -1.24030474 & -0.00592573 \\
\hline $\mathrm{C}$ & -9.72833909 & 0.03560286 & 0.31416900 \\
\hline $\mathrm{C}$ & -10.57767831 & -1.17551042 & 0.12064427 \\
\hline $\mathrm{C}$ & -10.46789202 & 1.26206932 & 0.73233238 \\
\hline $\mathrm{C}$ & -7.71570142 & -2.29923566 & 0.90892307 \\
\hline $\mathrm{C}$ & -6.89896968 & -3.42438348 & 0.83599115 \\
\hline $\mathrm{C}$ & -5.90774756 & -3.54062503 & -0.15259420 \\
\hline $\mathrm{C}$ & -5.78779393 & -2.49320718 & -1.08121818 \\
\hline $\mathrm{C}$ & -6.59977535 & -1.36711758 & -1.00466461 \\
\hline $\mathrm{C}$ & -10.46447094 & -1.98308574 & -1.01961088 \\
\hline $\mathrm{C}$ & -11.27871883 & -3.09964132 & -1.21546511 \\
\hline $\mathrm{C}$ & -12.23834495 & -3.43483041 & -0.25305427 \\
\hline $\mathrm{C}$ & -12.37642846 & -2.63343277 & 0.89040993 \\
\hline $\mathrm{C}$ & -11.56748832 & -1.52020986 & 1.06341181 \\
\hline $\mathrm{C}$ & -11.66928493 & 1.63279360 & 0.09502561 \\
\hline $\mathrm{C}$ & -12.37673887 & 2.76128461 & 0.48229682 \\
\hline $\mathrm{C}$ & -11.91554624 & 3.55229739 & 1.54552033 \\
\hline $\mathrm{C}$ & -10.73788651 & 3.19104806 & 2.21037586 \\
\hline $\mathrm{C}$ & -10.03161750 & 2.05949296 & 1.79957183 \\
\hline $\mathrm{C}$ & -4.94975775 & 4.70531722 & -0.39791595 \\
\hline $\mathrm{C}$ & -4.98452254 & -4.70240202 & -0.19450009 \\
\hline $\mathrm{C}$ & -5.43498415 & 6.02373348 & -0.41832518 \\
\hline $\mathrm{C}$ & -4.55386943 & 7.09659966 & -0.55040400 \\
\hline $\mathrm{C}$ & -3.18283265 & 6.88062247 & -0.67852862 \\
\hline $\mathrm{C}$ & -2.67142446 & 5.57157946 & -0.67658744 \\
\hline $\mathrm{C}$ & -3.56930215 & 4.50239589 & -0.52567038 \\
\hline $\mathrm{C}$ & -3.62362199 & -4.50202661 & -0.45959261 \\
\hline $\mathrm{C}$ & -2.70572533 & -5.56536316 & -0.49631134 \\
\hline $\mathrm{C}$ & -3.18240220 & -6.86456729 & -0.24910880 \\
\hline $\mathrm{C}$ & -4.53139746 & -7.07676392 & 0.02918362 \\
\hline $\mathrm{C}$ & -5.43010022 & -6.01114631 & 0.05689303 \\
\hline $\mathrm{C}$ & 7.08689661 & -3.46448667 & -0.90627561 \\
\hline $\mathrm{C}$ & 5.85803432 & -3.54282711 & -0.23014794 \\
\hline $\mathrm{C}$ & 5.49779241 & -2.46460437 & 0.59504233 \\
\hline $\mathrm{C}$ & 6.31952652 & -1.35014036 & 0.72118018 \\
\hline $\mathrm{C}$ & 7.54675010 & -1.26433426 & 0.03930475 \\
\hline
\end{tabular}




\begin{tabular}{|c|c|c|c|}
\hline $\mathrm{C}$ & 7.91046400 & -2.34922086 & -0.77771442 \\
\hline $\mathrm{C}$ & 8.37290348 & -0.02147888 & 0.12459149 \\
\hline $\mathrm{C}$ & 7.58170962 & 1.24030560 & -0.00594116 \\
\hline $\mathrm{C}$ & 9.72834083 & -0.03560759 & 0.31412763 \\
\hline $\mathrm{C}$ & 10.57768011 & 1.17550800 & 0.12061829 \\
\hline $\mathrm{C}$ & 10.46789403 & -1.26208045 & 0.73227199 \\
\hline $\mathrm{C}$ & 6.59977472 & 1.36713512 & -1.00467597 \\
\hline $\mathrm{C}$ & 5.78779276 & 2.49322580 & -1.08120875 \\
\hline $\mathrm{C}$ & 5.90774798 & 3.54062794 & -0.15256722 \\
\hline $\mathrm{C}$ & 6.89897227 & 3.42436999 & 0.83601400 \\
\hline $\mathrm{C}$ & 7.71570444 & 2.29922110 & 0.90892519 \\
\hline $\mathrm{C}$ & 10.46446729 & 1.98310284 & -1.01962252 \\
\hline $\mathrm{C}$ & 11.27871529 & 3.09966086 & -1.21546230 \\
\hline $\mathrm{C}$ & 12.23834717 & 3.43483257 & -0.25305114 \\
\hline $\mathrm{C}$ & 12.37643613 & 2.63341542 & 0.89039872 \\
\hline $\mathrm{C}$ & 11.56749573 & 1.52019051 & 1.06338621 \\
\hline $\mathrm{C}$ & 10.03162295 & -2.05951624 & 1.79950372 \\
\hline $\mathrm{C}$ & 10.73789185 & -3.19107755 & 2.21029081 \\
\hline $\mathrm{C}$ & 11.91554788 & -3.55232090 & 1.54542554 \\
\hline $\mathrm{C}$ & 12.37673713 & -2.76129600 & 0.48220954 \\
\hline $\mathrm{C}$ & 11.66928334 & -1.63279900 & 0.09495524 \\
\hline $\mathrm{C}$ & 4.94975623 & -4.70531093 & -0.39800650 \\
\hline $\mathrm{C}$ & 4.98452235 & 4.70240525 & -0.19445127 \\
\hline $\mathrm{C}$ & 5.43498262 & -6.02372686 & -0.41843525 \\
\hline $\mathrm{C}$ & 4.55386749 & -7.09659135 & -0.55052501 \\
\hline $\mathrm{C}$ & 3.18283019 & -6.88061263 & -0.67864129 \\
\hline $\mathrm{C}$ & 2.67142193 & -5.57156962 & -0.67668057 \\
\hline $\mathrm{C}$ & 3.56930010 & -4.50238801 & -0.52575285 \\
\hline $\mathrm{C}$ & 3.62362085 & 4.50203337 & -0.45954155 \\
\hline $\mathrm{C}$ & 2.70572348 & 5.56537009 & -0.49623964 \\
\hline $\mathrm{C}$ & 3.18240080 & 6.86457046 & -0.24901844 \\
\hline $\mathrm{C}$ & 4.53139707 & 7.07676334 & 0.02927190 \\
\hline $\mathrm{C}$ & 5.43010043 & 6.01114573 & 0.05696089 \\
\hline $\mathrm{C}$ & -0.75232080 & -4.21156326 & -1.44105505 \\
\hline $\mathrm{C}$ & 0.66528683 & -4.21046015 & -1.48234775 \\
\hline $\mathrm{C}$ & 1.24068645 & -5.28611825 & -0.84452499 \\
\hline S & -0.00440868 & -6.31923627 & -0.17002321 \\
\hline $\mathrm{C}$ & -1.29070210 & -5.28525441 & -0.76681733 \\
\hline $\mathrm{C}$ & -1.24068971 & 5.28613019 & -0.84444155 \\
\hline S & 0.00440824 & 6.31923960 & -0.16993184 \\
\hline $\mathrm{C}$ & 1.29069919 & 5.28526507 & -0.76674400 \\
\hline $\mathrm{C}$ & 0.75231507 & 4.21158300 & -1.44099397 \\
\hline $\mathrm{C}$ & -0.66529271 & 4.21048058 & -1.48228103 \\
\hline $\mathrm{O}$ & -12.68412090 & 4.63667882 & 1.85549331 \\
\hline
\end{tabular}




\begin{tabular}{|c|c|c|c|}
\hline $\mathrm{C}$ & -12.26751441 & 5.47144891 & 2.92405415 \\
\hline $\mathrm{O}$ & -13.08499510 & -4.50210426 & -0.33524857 \\
\hline $\mathrm{C}$ & -12.99287487 & -5.34641475 & -1.47139736 \\
\hline $\mathrm{O}$ & 12.68412190 & -4.63670754 & 1.85538197 \\
\hline $\mathrm{C}$ & 12.26751965 & -5.47148839 & 2.92393604 \\
\hline $\mathrm{O}$ & 13.08499806 & 4.50210681 & -0.33523218 \\
\hline $\mathrm{C}$ & 12.99287196 & 5.34643692 & -1.47136592 \\
\hline $\mathrm{H}$ & -4.56796443 & 2.50821671 & 1.15570455 \\
\hline $\mathrm{H}$ & -7.38122256 & 4.26949015 & -1.57462628 \\
\hline $\mathrm{H}$ & -8.84243642 & 2.30691208 & -1.33248580 \\
\hline $\mathrm{H}$ & -6.00796384 & 0.52866178 & 1.35986550 \\
\hline $\mathrm{H}$ & -8.45305478 & -2.22514675 & 1.70217354 \\
\hline $\mathrm{H}$ & -6.99892883 & -4.20423413 & 1.58631092 \\
\hline $\mathrm{H}$ & -5.05306790 & -2.56811953 & -1.87839665 \\
\hline $\mathrm{H}$ & -6.47133927 & -0.56681757 & -1.72789406 \\
\hline $\mathrm{H}$ & -9.72641473 & -1.73311899 & -1.77527918 \\
\hline $\mathrm{H}$ & -11.15985658 & -3.69083013 & -2.11644223 \\
\hline $\mathrm{H}$ & -13.12880934 & -2.90308722 & 1.62519911 \\
\hline $\mathrm{H}$ & -11.69456325 & -0.90409250 & 1.94886216 \\
\hline $\mathrm{H}$ & -12.04578375 & 1.02568830 & -0.72323633 \\
\hline $\mathrm{H}$ & -13.29472291 & 3.05094484 & -0.01980032 \\
\hline $\mathrm{H}$ & -10.36543119 & 3.77305437 & 3.04581894 \\
\hline $\mathrm{H}$ & -9.12074243 & 1.79014952 & 2.32498988 \\
\hline $\mathrm{H}$ & -6.49927078 & 6.20558466 & -0.29829026 \\
\hline $\mathrm{H}$ & -4.93944158 & 8.11271851 & -0.55517909 \\
\hline $\mathrm{H}$ & -2.50908560 & 7.72331168 & -0.80683708 \\
\hline $\mathrm{H}$ & -3.18051287 & 3.48906392 & -0.50914913 \\
\hline $\mathrm{H}$ & -3.25968978 & -3.48777194 & -0.58721130 \\
\hline $\mathrm{H}$ & -2.49931463 & -7.70872364 & -0.28816554 \\
\hline $\mathrm{H}$ & -4.88790943 & -8.08687958 & 0.21284439 \\
\hline $\mathrm{H}$ & -6.48295683 & -6.19240677 & 0.25388769 \\
\hline $\mathrm{H}$ & 7.38121833 & -4.26946893 & -1.57471729 \\
\hline $\mathrm{H}$ & 4.56796667 & -2.50823003 & 1.15564249 \\
\hline $\mathrm{H}$ & 6.00796701 & -0.52867819 & 1.35982585 \\
\hline $\mathrm{H}$ & 8.84243330 & -2.30689454 & -1.33255453 \\
\hline $\mathrm{H}$ & 6.47133715 & 0.56684720 & -1.72791858 \\
\hline $\mathrm{H}$ & 5.05306501 & 2.56815127 & -1.87838441 \\
\hline $\mathrm{H}$ & 6.99893290 & 4.20420798 & 1.58634674 \\
\hline $\mathrm{H}$ & 8.45305938 & 2.22511888 & 1.7021729 \\
\hline $\mathrm{H}$ & 9.72640669 & 1.73314964 & -1.77529101 \\
\hline $\mathrm{H}$ & 11.15984864 & 3.69086498 & -2.11642881 \\
\hline $\mathrm{H}$ & 13.12882135 & 2.90305660 & 1.62518832 \\
\hline $\mathrm{H}$ & 11.69457484 & 0.90405809 & 1.9488254 \\
\hline $\mathrm{H}$ & 9.12075068 & -1.79017758 & 2.32492911 \\
\hline
\end{tabular}




$\begin{array}{lrrr}\mathrm{H} & 10.36543913 & -3.77309334 & 3.04572844 \\ \mathrm{H} & 13.29471836 & -3.05095173 & -0.01989526 \\ \mathrm{H} & 12.04577962 & -1.02568435 & -0.72330097 \\ \mathrm{H} & 6.49926972 & -6.20557963 & -0.29840679 \\ \mathrm{H} & 4.93943974 & -8.11271009 & -0.55531518 \\ \mathrm{H} & 2.50908266 & -7.72330014 & -0.80695826 \\ \mathrm{H} & 3.18051075 & -3.48905630 & -0.50921652 \\ \mathrm{H} & 3.25968877 & 3.48778051 & -0.58717491 \\ \mathrm{H} & 2.49931270 & 7.70872713 & -0.28805896 \\ \mathrm{H} & 4.88790925 & 8.08687629 & 0.21294728 \\ \mathrm{H} & 6.48295773 & 6.19240349 & 0.25395433 \\ \mathrm{H} & -1.36232518 & -3.45408163 & -1.92098801 \\ \mathrm{H} & 1.24967410 & -3.45526231 & -1.99701411 \\ \mathrm{H} & 1.36231742 & 3.45410751 & -1.92093919 \\ \mathrm{H} & -1.24968208 & 3.45528951 & -1.99695496 \\ \mathrm{H} & -13.01268657 & 6.26631859 & 2.99109655 \\ \mathrm{H} & -12.23523105 & 4.92435397 & 3.87561830 \\ \mathrm{H} & -11.28146399 & 5.91413050 & 2.73047132 \\ \mathrm{H} & -13.74571830 & -6.12387419 & -1.32864117 \\ \mathrm{H} & -13.20792149 & -4.80013614 & -2.39944016 \\ \mathrm{H} & -12.00132397 & -5.81169861 & -1.54993354 \\ \mathrm{H} & 13.01269097 & -6.26635987 & 2.99096619 \\ \mathrm{H} & 12.23524190 & -4.92440349 & 3.87550614 \\ \mathrm{H} & 11.28146765 & -5.91416653 & 2.73035357 \\ \mathrm{H} & 13.74571687 & 6.12389329 & -1.32860075 \\ \mathrm{H} & 13.20791271 & 4.80017406 & -2.39941934 \\ \mathrm{H} & 12.00132095 & 5.81172295 & -1.54988833\end{array}$

\section{(M,P)-(out,out)-TPEMCS}

$\begin{array}{lrrr}\text { C } & -5.69050236 & 2.54771647 & -0.72686925 \\ C & -5.89946520 & 3.52798305 & 0.25833357 \\ C & -6.98944401 & 3.35255537 & 1.12774104 \\ C & -7.82195847 & 2.24116694 & 1.02757962 \\ C & -7.59959771 & 1.25019382 & 0.05629899 \\ C & -6.51227462 & 1.43008441 & -0.81693124 \\ C & -8.41684143 & 0.00000042 & -0.00000089 \\ C & -7.59959650 & -1.25019200 & -0.05630409 \\ C & -9.78454941 & -0.00000059 & 0.00000248 \\ C & -10.58459466 & -1.23855913 & 0.22687282 \\ C & -10.58459815 & 1.23855640 & -0.22686343 \\ C & -7.82196064 & -2.24116608 & -1.02758299 \\ C & -6.98944597 & -3.35255410 & -1.12774690 \\ C & -5.89946361 & -3.52798064 & -0.25834365 \\ C & -5.69049728 & -2.54771296 & 0.72685740\end{array}$




\begin{tabular}{|c|c|c|c|}
\hline $\mathrm{C}$ & -6.51226960 & -1.43008118 & 0.81692166 \\
\hline $\mathrm{C}$ & -10.30404525 & -2.11223537 & 1.28674302 \\
\hline $\mathrm{C}$ & -11.06827044 & -3.25635283 & 1.52071427 \\
\hline $\mathrm{C}$ & -12.14723978 & -3.55264650 & 0.67964792 \\
\hline $\mathrm{C}$ & -12.45303755 & -2.68527266 & -0.38014781 \\
\hline $\mathrm{C}$ & -11.69074640 & -1.54578917 & -0.59099815 \\
\hline $\mathrm{C}$ & -11.69074725 & 1.54578276 & 0.59101258 \\
\hline $\mathrm{C}$ & -12.45304179 & 2.68526483 & 0.38016713 \\
\hline $\mathrm{C}$ & -12.14725042 & 3.55264088 & -0.67962863 \\
\hline $\mathrm{C}$ & -11.06828393 & 3.25635086 & -1.52069999 \\
\hline $\mathrm{C}$ & -10.30405509 & 2.11223482 & -1.28673353 \\
\hline $\mathrm{C}$ & -4.97572284 & 4.68211464 & 0.39365017 \\
\hline $\mathrm{C}$ & -4.97572152 & -4.68211222 & -0.39366224 \\
\hline $\mathrm{C}$ & -5.43488488 & 5.95477708 & 0.77481068 \\
\hline $\mathrm{C}$ & -4.53967874 & 7.01371759 & 0.91888231 \\
\hline $\mathrm{C}$ & -3.17881934 & 6.83422855 & 0.67817108 \\
\hline $\mathrm{C}$ & -2.68992395 & 5.57389170 & 0.29335791 \\
\hline $\mathrm{C}$ & -3.60272177 & 4.51341416 & 0.16757622 \\
\hline $\mathrm{C}$ & -3.60272012 & -4.51341205 & -0.16758994 \\
\hline $\mathrm{C}$ & -2.68992254 & -5.57388974 & -0.29337227 \\
\hline $\mathrm{C}$ & -3.17881839 & -6.83422643 & -0.67818525 \\
\hline $\mathrm{C}$ & -4.53967806 & -7.01371515 & -0.91889526 \\
\hline $\mathrm{C}$ & -5.43488398 & -5.95477464 & -0.77482241 \\
\hline $\mathrm{C}$ & 6.98944714 & -3.35255362 & -1.12774508 \\
\hline $\mathrm{C}$ & 5.89946488 & -3.52798015 & -0.25834173 \\
\hline $\mathrm{C}$ & 5.69049834 & -2.54771229 & 0.72685909 \\
\hline $\mathrm{C}$ & 6.51227028 & -1.43008022 & 0.81692295 \\
\hline $\mathrm{C}$ & 7.59959692 & -1.25019091 & -0.05630309 \\
\hline $\mathrm{C}$ & 7.82196149 & -2.24116526 & -1.02758159 \\
\hline $\mathrm{C}$ & 8.41684149 & 0.00000183 & -0.00000060 \\
\hline $\mathrm{C}$ & 7.59959734 & 1.25019493 & 0.05629953 \\
\hline $\mathrm{C}$ & 9.78454946 & 0.00000066 & 0.00000280 \\
\hline $\mathrm{C}$ & 10.58459878 & 1.23855714 & -0.22686406 \\
\hline $\mathrm{C}$ & 10.58459407 & -1.23855807 & 0.22687408 \\
\hline $\mathrm{C}$ & 6.51227388 & 1.43008518 & -0.81693034 \\
\hline $\mathrm{C}$ & 5.69050136 & 2.54771699 & -0.72686819 \\
\hline $\mathrm{C}$ & 5.89946408 & 3.52798371 & 0.25833451 \\
\hline $\mathrm{C}$ & 6.98944311 & 3.35255629 & 1.12774177 \\
\hline $\mathrm{C}$ & 7.82195789 & 2.24116814 & 1.02758020 \\
\hline $\mathrm{C}$ & 10.30405758 & 2.11223377 & -1.28673616 \\
\hline $\mathrm{C}$ & 11.06828700 & 3.25634918 & -1.52070354 \\
\hline $\mathrm{C}$ & 12.14725243 & 3.55264036 & -0.67963124 \\
\hline $\mathrm{C}$ & 12.45304210 & 2.68526607 & 0.38016643 \\
\hline $\mathrm{C}$ & 11.69074693 & 1.54578458 & 0.59101284 \\
\hline
\end{tabular}




\begin{tabular}{|c|c|c|c|}
\hline $\mathrm{C}$ & 10.30404335 & -2.11223396 & 1.28674421 \\
\hline $\mathrm{C}$ & 11.06826779 & -3.25635174 & 1.52071636 \\
\hline $\mathrm{C}$ & 12.14723756 & -3.55264620 & 0.67965084 \\
\hline $\mathrm{C}$ & 12.45303649 & -2.68527283 & -0.38014493 \\
\hline $\mathrm{C}$ & 11.69074619 & -1.54578896 & -0.59099610 \\
\hline $\mathrm{C}$ & 4.97572304 & -4.68211196 & -0.39366007 \\
\hline $\mathrm{C}$ & 4.97572150 & 4.68211514 & 0.39365115 \\
\hline $\mathrm{C}$ & 5.43488583 & -5.95477443 & -0.77481959 \\
\hline $\mathrm{C}$ & 4.53968010 & -7.01371515 & -0.91889243 \\
\hline $\mathrm{C}$ & 3.17882031 & -6.83422638 & -0.67818308 \\
\hline $\mathrm{C}$ & 2.68992415 & -5.57388963 & -0.29337076 \\
\hline $\mathrm{C}$ & 3.60272155 & -4.51341182 & -0.16758838 \\
\hline $\mathrm{C}$ & 3.60272045 & 4.51341457 & 0.16757696 \\
\hline $\mathrm{C}$ & 2.68992250 & 5.57389202 & 0.29335851 \\
\hline $\mathrm{C}$ & 3.17881766 & 6.83422887 & 0.67817180 \\
\hline $\mathrm{C}$ & 4.53967701 & 7.01371806 & 0.91888331 \\
\hline $\mathrm{C}$ & 5.43488329 & 5.95477766 & 0.77481181 \\
\hline $\mathrm{C}$ & -0.70907639 & -4.40889954 & 0.82637677 \\
\hline $\mathrm{C}$ & 0.70907734 & -4.40889951 & 0.82637716 \\
\hline $\mathrm{C}$ & 1.26630938 & -5.33238102 & -0.02974636 \\
\hline S & 0.00000097 & -6.21755629 & -0.85970099 \\
\hline $\mathrm{C}$ & -1.26630794 & -5.33238102 & -0.02974708 \\
\hline $\mathrm{C}$ & -1.26630934 & 5.33238298 & 0.02973263 \\
\hline S & -0.00000083 & 6.21755745 & 0.85968798 \\
\hline $\mathrm{C}$ & 1.26630798 & 5.33238309 & 0.02973291 \\
\hline $\mathrm{C}$ & 0.70907637 & 4.40890198 & -0.82639126 \\
\hline $\mathrm{C}$ & -0.70907743 & 4.40890187 & -0.82639140 \\
\hline $\mathrm{O}$ & -12.95959337 & 4.64175807 & -0.80875914 \\
\hline $\mathrm{C}$ & -12.70060673 & 5.55067225 & -1.86671073 \\
\hline $\mathrm{O}$ & -12.95957924 & -4.64176574 & 0.80878316 \\
\hline $\mathrm{C}$ & -12.70059022 & -5.55067394 & 1.86673930 \\
\hline $\mathrm{O}$ & 12.95957665 & -4.64176556 & 0.80878728 \\
\hline $\mathrm{C}$ & 12.70058233 & -5.55067685 & 1.86673948 \\
\hline $\mathrm{O}$ & 12.95959560 & 4.64175731 & -0.80876233 \\
\hline $\mathrm{C}$ & 12.70061355 & 5.55066722 & -1.86671873 \\
\hline $\mathrm{H}$ & -4.87798099 & 2.66834284 & -1.43802520 \\
\hline $\mathrm{H}$ & -7.16275713 & 4.07418710 & 1.92151599 \\
\hline $\mathrm{H}$ & -8.64400093 & 2.12334186 & 1.72689846 \\
\hline $\mathrm{H}$ & -6.31250360 & 0.68127660 & -1.57831187 \\
\hline $\mathrm{H}$ & -8.64400617 & -2.12334216 & -1.72689839 \\
\hline $\mathrm{H}$ & -7.16276205 & -4.07418649 & -1.92152060 \\
\hline $\mathrm{H}$ & -4.87797328 & -2.66833834 & 1.43801049 \\
\hline $\mathrm{H}$ & -6.31249582 & -0.68127266 & 1.57830087 \\
\hline $\mathrm{H}$ & -9.47217782 & -1.89329880 & 1.94878947 \\
\hline
\end{tabular}


$\mathrm{H}$

$\mathrm{H}$

$\mathrm{H}$

$\mathrm{H}$

$\mathrm{H}$

$\mathrm{H}$

$\mathrm{H}$

$\mathrm{H}$

$\mathrm{H}$

$\mathrm{H}$

$\mathrm{H}$

$\mathrm{H}$

$\mathrm{H}$

$\mathrm{H}$

$\mathrm{H}$

$\mathrm{H}$

$\mathrm{H}$

$\mathrm{H}$

$\mathrm{H}$

$\mathrm{H}$

$\mathrm{H}$

$\mathrm{H}$

$\mathrm{H}$

$\mathrm{H}$

$\mathrm{H}$

$\mathrm{H}$

$\mathrm{H}$

$\mathrm{H}$

$\mathrm{H}$

$\mathrm{H}$

$\mathrm{H}$

$\mathrm{H}$

$\mathrm{H}$

$\mathrm{H}$

$\mathrm{H}$

$\mathrm{H}$

$\mathrm{H}$

$\mathrm{H}$

$\mathrm{H}$

$\mathrm{H}$

$\mathrm{H}$

$\mathrm{H}$

$\mathrm{H}$

$\mathrm{H}$
$-10.81805131$

$-13.29638231$

$-11.94775424$

$-11.94775006$

$-13.29638437$

$-10.81807015$

$-9.47218994$

$-6.49622468$

$-4.90855323$

$-2.49620188$

$-3.22463905$

$-3.22463687$

$-2.49620117$

$-4.90855297$

$-6.49622399$

7.16276343

4.87797435

6.31249640

8.64400691

6.31250291

4.87797985

7.16275618

8.64400040

9.47219333

10.81807443

13.29638395

11.94774853

9.47217549

10.81804787

13.29638151

11.94775488

6.49622595

4.90855523

2.49620321

3.22463813

3.22463781

2.49620009

4.90855136

6.49622304

$-1.30649405$

1.30649463

1.30649399

$-1.30649483$

$-13.45609832$
$-3.89897073$

$-2.92543639$

$-0.87875171$

0.87874358

2.92542581

3.89897037

1.89330099

6.11724248

7.99499885

7.67467690

3.52246868

$-3.52246666$

$-7.67467495$

$-7.99499631$

$-6.11724021$

$-4.07418627$

$-2.66833775$

$-0.68127149$

$-2.12334126$

0.68127736

2.66834306

4.07418800

2.12334334

1.89329899

3.89896737

2.92542786

0.87874674

$-1.89329679$

$-3.89896915$

$-2.92543724$

$-0.87875193$

$-6.11723989$

$-7.99499636$

$-7.67467500$

$-3.52246638$

3.52246908

7.67467722

7.99499938

6.11724328

$-3.76807851$

$-3.76807844$

3.76808133

3.76808114

6.33385071
2.35713040

$-1.02032416$

$-1.40884559$

1.40886018

1.02034738

$-2.35711647$

$-1.94878372$

0.93896070

1.20556836

0.76892292

$-0.06178977$

0.06177546

$-0.76893780$

$-1.20558118$

$-0.93897103$

$-1.92151851$

1.43801221

1.57830192

$-1.72689705$

$-1.57831096$

$-1.43802408$

1.92151674

1.72689894

$-1.94878719$

$-2.35712140$

1.02034735

1.40886191

1.94878996

2.35713262

$-1.02032070$

$-1.40884361$

$-0.93896780$

$-1.20557785$

$-0.76893565$

0.06177652

$-0.06178910$

0.76892356

1.20556946

0.93896191

1.46570267

1.46570337

$-1.46571759$

$-1.46571785$

$-1.78041586$ 


$\begin{array}{lrrr}\mathrm{H} & -11.70189886 & 5.99871244 & -1.77927106 \\ \mathrm{H} & -12.79213523 & 5.06554773 & -2.84762251 \\ \mathrm{H} & -13.45608255 & -6.33385241 & 1.78045110 \\ \mathrm{H} & -11.70188288 & -5.99871541 & 1.77929940 \\ \mathrm{H} & -12.79211550 & -5.06554362 & 2.84764847 \\ \mathrm{H} & 13.45607228 & -6.33385749 & 1.78045007 \\ \mathrm{H} & 12.79210708 & -5.06555051 & 2.84765070 \\ \mathrm{H} & 11.70187368 & -5.99871456 & 1.77929571 \\ \mathrm{H} & 13.45610673 & 6.33384436 & -1.78042562 \\ \mathrm{H} & 12.79214328 & 5.06553794 & -2.84762802 \\ \mathrm{H} & 11.70190654 & 5.99871011 & -1.77928353\end{array}$

\section{(M,M)-(in,out)-TPEMCS}

\begin{tabular}{|c|c|c|c|}
\hline $\mathrm{C}$ & -4.67726278 & -2.07054195 & 0.81417006 \\
\hline $\mathrm{C}$ & -5.22383831 & -3.05585682 & 1.65377347 \\
\hline $\mathrm{C}$ & -6.62111120 & -3.06359848 & 1.81352505 \\
\hline $\mathrm{C}$ & -7.42816937 & -2.11648404 & 1.19329466 \\
\hline $\mathrm{C}$ & -6.88092553 & -1.11715342 & 0.37019309 \\
\hline $\mathrm{C}$ & -5.48533727 & -1.12348141 & 0.19208001 \\
\hline $\mathrm{C}$ & -7.73005250 & -0.02834010 & -0.20429252 \\
\hline $\mathrm{C}$ & -7.16129448 & 1.34249971 & -0.03129414 \\
\hline $\mathrm{C}$ & -8.92708144 & -0.27003969 & -0.82539203 \\
\hline $\mathrm{C}$ & -9.91936027 & 0.80826033 & -1.10184481 \\
\hline $\mathrm{C}$ & -9.33517838 & -1.63288822 & -1.27243915 \\
\hline $\mathrm{C}$ & -7.13148413 & 2.27483084 & -1.08404058 \\
\hline $\mathrm{C}$ & -6.48376996 & 3.49958711 & -0.94657807 \\
\hline $\mathrm{C}$ & -5.82337760 & 3.84007092 & 0.24491932 \\
\hline $\mathrm{C}$ & -5.88445201 & 2.92805136 & 1.31073898 \\
\hline $\mathrm{C}$ & -6.53705636 & 1.70808085 & 1.17521076 \\
\hline $\mathrm{C}$ & -10.29003518 & 1.73548764 & -0.11773530 \\
\hline $\mathrm{C}$ & -11.24035973 & 2.72805933 & -0.36090625 \\
\hline $\mathrm{C}$ & -11.84789453 & 2.81244929 & -1.61927794 \\
\hline $\mathrm{C}$ & -11.50135974 & 1.88640398 & -2.61482507 \\
\hline $\mathrm{C}$ & -10.56366824 & 0.89837585 & -2.35246512 \\
\hline $\mathrm{C}$ & -10.63540927 & -2.11863094 & -1.02592717 \\
\hline $\mathrm{C}$ & -11.02993605 & -3.37951245 & -1.44861972 \\
\hline $\mathrm{C}$ & -10.13775411 & -4.19665224 & -2.15954690 \\
\hline $\mathrm{C}$ & -8.84906309 & -3.72663938 & -2.43850066 \\
\hline $\mathrm{C}$ & -8.46450792 & -2.46047257 & -1.99548688 \\
\hline $\mathrm{C}$ & -4.36581792 & -4.02765381 & 2.37870169 \\
\hline $\mathrm{C}$ & -4.99903269 & 5.07023161 & 0.35177426 \\
\hline $\mathrm{C}$ & -4.74924940 & -4.50488654 & 3.64475471 \\
\hline $\mathrm{C}$ & -3.95649262 & -5.42143547 & 4.33122712 \\
\hline $\mathrm{C}$ & -2.76105217 & -5.87233438 & 3.78006982 \\
\hline
\end{tabular}




\begin{tabular}{|c|c|c|c|}
\hline $\mathrm{C}$ & -2.34610777 & -5.41319433 & 2.51682658 \\
\hline $\mathrm{C}$ & -3.15893432 & -4.49481501 & 1.83269379 \\
\hline $\mathrm{C}$ & -3.70083092 & 4.96703665 & 0.86508520 \\
\hline $\mathrm{C}$ & -2.83610827 & 6.07098271 & 0.93780119 \\
\hline $\mathrm{C}$ & -3.30938208 & 7.31701431 & 0.49271609 \\
\hline $\mathrm{C}$ & -4.60063512 & 7.43369302 & -0.02094247 \\
\hline $\mathrm{C}$ & -5.44224833 & 6.32357007 & -0.10038455 \\
\hline $\mathrm{C}$ & 6.70257660 & 3.41351347 & 1.25796637 \\
\hline $\mathrm{C}$ & 5.43417578 & 3.66466992 & 0.70879042 \\
\hline $\mathrm{C}$ & 4.90485446 & 2.70877800 & -0.17432218 \\
\hline $\mathrm{C}$ & 5.61399503 & 1.55778532 & -0.49958179 \\
\hline $\mathrm{C}$ & 6.89371294 & 1.31445800 & 0.03015889 \\
\hline $\mathrm{C}$ & 7.41291012 & 2.26266424 & 0.93052590 \\
\hline $\mathrm{C}$ & 7.63521811 & 0.07007323 & -0.32673657 \\
\hline $\mathrm{C}$ & 6.80254046 & -1.17114122 & -0.36983158 \\
\hline $\mathrm{C}$ & 8.97197028 & 0.05677641 & -0.62640956 \\
\hline $\mathrm{C}$ & 9.76213510 & -1.20621674 & -0.70163007 \\
\hline $\mathrm{C}$ & 9.74603255 & 1.30003799 & -0.91277104 \\
\hline $\mathrm{C}$ & 5.95637853 & -1.52851745 & 0.69408867 \\
\hline $\mathrm{C}$ & 5.17968729 & -2.68338358 & 0.64351810 \\
\hline $\mathrm{C}$ & 5.18960236 & -3.51928508 & -0.48657416 \\
\hline $\mathrm{C}$ & 6.00365189 & -3.13660271 & -1.56706004 \\
\hline $\mathrm{C}$ & 6.79415147 & -1.99443646 & -1.50830811 \\
\hline $\mathrm{C}$ & 9.67960080 & -2.18633833 & 0.29772975 \\
\hline $\mathrm{C}$ & 10.43983859 & -3.35537026 & 0.24866998 \\
\hline $\mathrm{C}$ & 11.31332369 & -3.56966125 & -0.82378788 \\
\hline $\mathrm{C}$ & 11.42151933 & -2.59617143 & -1.82855925 \\
\hline $\mathrm{C}$ & 10.66754203 & -1.43399440 & -1.75786624 \\
\hline $\mathrm{C}$ & 9.27851234 & 2.26076942 & -1.82017054 \\
\hline $\mathrm{C}$ & 10.01281493 & 3.40859078 & -2.12152077 \\
\hline $\mathrm{C}$ & 11.25230289 & 3.61943588 & -1.50605405 \\
\hline $\mathrm{C}$ & 11.74503358 & 2.66413001 & -0.60410841 \\
\hline $\mathrm{C}$ & 11.00786967 & 1.52234615 & -0.32559651 \\
\hline $\mathrm{C}$ & 4.66567608 & 4.89137599 & 1.03680963 \\
\hline $\mathrm{C}$ & 4.39653669 & -4.77467619 & -0.53956124 \\
\hline $\mathrm{C}$ & 5.28310299 & 6.15124757 & 1.11424523 \\
\hline $\mathrm{C}$ & 4.52393853 & 7.29710116 & 1.35203114 \\
\hline $\mathrm{C}$ & 3.14238843 & 7.21400281 & 1.52319152 \\
\hline $\mathrm{C}$ & 2.50455206 & 5.96417632 & 1.47364940 \\
\hline $\mathrm{C}$ & 3.27988515 & 4.82033623 & 1.22963385 \\
\hline $\mathrm{C}$ & 3.15477345 & -4.88973445 & 0.10560179 \\
\hline $\mathrm{C}$ & 2.42150735 & -6.08784854 & 0.08908541 \\
\hline $\mathrm{C}$ & 2.94383515 & -7.18672717 & -0.61739169 \\
\hline $\mathrm{C}$ & 4.17342024 & -7.08695662 & -1.26089286 \\
\hline
\end{tabular}




\begin{tabular}{|c|c|c|c|}
\hline $\mathrm{C}$ & 4.89785354 & -5.89787010 & -1.22085675 \\
\hline $\mathrm{C}$ & -1.00721914 & 4.87306532 & 2.25883220 \\
\hline $\mathrm{C}$ & 0.40523110 & 4.83771215 & 2.39136327 \\
\hline $\mathrm{C}$ & 1.05391703 & 5.79948345 & 1.65343099 \\
\hline S & -0.10809932 & 6.77501573 & 0.77682087 \\
\hline $\mathrm{C}$ & -1.46251224 & 5.85587083 & 1.40880841 \\
\hline $\mathrm{C}$ & -1.09210535 & -5.92738385 & 1.94690649 \\
\hline $\mathrm{S}$ & 0.00819064 & -4.92308983 & 1.02003500 \\
\hline $\mathrm{C}$ & 1.15105429 & -6.23813435 & 0.81311433 \\
\hline $\mathrm{C}$ & 0.67614616 & -7.37346953 & 1.43039516 \\
\hline $\mathrm{C}$ & -0.57895205 & -7.19831430 & 2.06850928 \\
\hline $\mathrm{O}$ & -10.62102666 & -5.41544682 & -2.53831998 \\
\hline $\mathrm{C}$ & -9.76109437 & -6.28347426 & -3.25923996 \\
\hline $\mathrm{O}$ & -12.78443322 & 3.74061424 & -1.97151163 \\
\hline $\mathrm{C}$ & -13.17332029 & 4.70250803 & -1.00415711 \\
\hline $\mathrm{O}$ & 12.05331584 & 4.70372941 & -1.71987158 \\
\hline $\mathrm{C}$ & 11.60762414 & 5.69806822 & -2.62813946 \\
\hline $\mathrm{O}$ & 12.10014967 & -4.67414538 & -0.97867623 \\
\hline $\mathrm{C}$ & 12.03164080 & -5.69046754 & 0.00870535 \\
\hline $\mathrm{H}$ & -3.60073593 & -2.01663572 & 0.67802209 \\
\hline $\mathrm{H}$ & -7.08674622 & -3.82736827 & 2.42963478 \\
\hline $\mathrm{H}$ & -8.50142296 & -2.14449265 & 1.35224372 \\
\hline $\mathrm{H}$ & -5.02507773 & -0.35774840 & -0.42553671 \\
\hline $\mathrm{H}$ & -7.59393774 & 2.02014241 & -2.03226082 \\
\hline $\mathrm{H}$ & -6.43500368 & 4.17632115 & -1.79577297 \\
\hline $\mathrm{H}$ & -5.40901192 & 3.17981535 & 2.25503541 \\
\hline $\mathrm{H}$ & -6.54994040 & 1.01579643 & 2.01178400 \\
\hline $\mathrm{H}$ & -9.82934855 & 1.67947640 & 0.86362204 \\
\hline $\mathrm{H}$ & -11.50032467 & 3.41855134 & 0.43341902 \\
\hline $\mathrm{H}$ & -11.98548538 & 1.96066576 & -3.58375605 \\
\hline $\mathrm{H}$ & -10.31448830 & 0.18295811 & -3.13089076 \\
\hline $\mathrm{H}$ & -11.34183994 & -1.49516838 & -0.48540319 \\
\hline $\mathrm{H}$ & -12.02777987 & -3.75555459 & -1.24456145 \\
\hline $\mathrm{H}$ & -8.14310588 & -4.32830307 & -2.99957476 \\
\hline $\mathrm{H}$ & -7.46317677 & -2.10738477 & -2.22148280 \\
\hline $\mathrm{H}$ & -5.65840755 & -4.13254731 & 4.10639250 \\
\hline $\mathrm{H}$ & -4.26185974 & -5.76867258 & 5.31480768 \\
\hline $\mathrm{H}$ & -2.12847492 & -6.55983416 & 4.33322229 \\
\hline $\mathrm{H}$ & -2.86636163 & -4.17562176 & 0.83645314 \\
\hline $\mathrm{H}$ & -3.32883298 & 3.98756075 & 1.14675784 \\
\hline $\mathrm{H}$ & -2.66818671 & 8.19250981 & 0.55196755 \\
\hline $\mathrm{H}$ & -4.95590749 & 8.40341822 & -0.35956026 \\
\hline $\mathrm{H}$ & -6.44720143 & 6.42738030 & -0.50035802 \\
\hline $\mathrm{H}$ & 7.12405078 & 4.11454468 & 1.97379567 \\
\hline
\end{tabular}




$\begin{array}{lrrr}\mathrm{H} & 3.93592637 & 2.88448447 & -0.63338984 \\ \mathrm{H} & 5.17852930 & 0.84122243 & -1.18991058 \\ \mathrm{H} & 8.38480938 & 2.08933106 & 1.38113296 \\ \mathrm{H} & 5.92550363 & -0.90334733 & 1.58244506 \\ \mathrm{H} & 4.57917702 & -2.95523534 & 1.50731374 \\ \mathrm{H} & 6.00089339 & -3.73028922 & -2.47672299 \\ \mathrm{H} & 7.41292933 & -1.72682074 & -2.35929010 \\ \mathrm{H} & 9.00913143 & -2.03314693 & 1.13737708 \\ \mathrm{H} & 10.34669805 & -4.08115413 & 1.04851836 \\ \mathrm{H} & 12.10739093 & -2.77336934 & -2.65131167 \\ \mathrm{H} & 10.77174512 & -0.68612925 & -2.53867847 \\ \mathrm{H} & 8.31925861 & 2.10890053 & -2.30528436 \\ \mathrm{H} & 9.61362145 & 4.12024681 & -2.83532722 \\ \mathrm{H} & 12.71083440 & 2.83857622 & -0.13985021 \\ \mathrm{H} & 11.40917949 & 0.78667083 & 0.36563687 \\ \mathrm{H} & 6.35361654 & 6.23486255 & 0.94864503 \\ \mathrm{H} & 5.01329406 & 8.26650250 & 1.39725162 \\ \mathrm{H} & 2.55914388 & 8.11003711 & 1.71666493 \\ \mathrm{H} & 2.78393554 & 3.85619644 & 1.17659883 \\ \mathrm{H} & 2.75312891 & -4.03316099 & 0.63973217 \\ \mathrm{H} & 2.37353535 & -8.10895981 & -0.67092443 \\ \mathrm{H} & 4.57243686 & -7.94420593 & -1.79683551 \\ \mathrm{H} & 5.87183489 & -5.84579921 & -1.69755498 \\ \mathrm{H} & -1.67341568 & 4.19531368 & 2.78154856 \\ \mathrm{H} & 0.93516676 & 4.13217179 & 3.02250175 \\ \mathrm{H} & 1.23181445 & -8.30483451 & 1.44807619 \\ \mathrm{H} & -1.10268982 & -7.99173478 & 2.59094768 \\ \mathrm{H} & -10.34135601 & -7.18783315 & -3.45160429 \\ \mathrm{H} & -9.45090103 & -5.84194913 & -4.21562435 \\ \mathrm{H} & -8.86748481 & -6.54279021 & -2.67623126 \\ \mathrm{H} & -13.91295344 & 5.33817721 & -1.49450479 \\ \mathrm{H} & -13.62902916 & 4.22955564 & -0.12404478 \\ \mathrm{H} & -12.32294705 & 5.31776525 & -0.68154864 \\ \mathrm{H} & 12.38708894 & 6.46214863 & -2.64185794 \\ \mathrm{H} & 1.48014910 & 5.29250757 & -3.64061466 \\ \mathrm{H} & 6.66212780 & 6.15025886 & -2.30057386 \\ \mathrm{H} & -6.46852659 & -0.31375967 \\ \mathrm{H} & -5.31747616 & 0.99495166 \\ \mathrm{H} & -6.11405498 & 0.08330241\end{array}$

\section{(M,P)-(in,out)-TPEMCS}

$\begin{array}{cccc}\text { C } & -5.11980945 & 2.48887174 & -0.41842616 \\ \mathrm{C} & -5.45742116 & 3.47550986 & 0.52302637 \\ \mathrm{C} & -6.56554293 & 3.22688813 & 1.35284427\end{array}$




\begin{tabular}{|c|c|c|c|}
\hline $\mathrm{C}$ & -7.33823088 & 2.08172446 & 1.20454840 \\
\hline $\mathrm{C}$ & -7.03688163 & 1.12214864 & 0.22323830 \\
\hline $\mathrm{C}$ & -5.88486440 & 1.33213434 & -0.55539805 \\
\hline $\mathrm{C}$ & -7.89571668 & -0.08653155 & 0.03760343 \\
\hline $\mathrm{C}$ & -7.16291424 & -1.38485312 & -0.01366467 \\
\hline $\mathrm{C}$ & -9.25762656 & -0.00184505 & -0.08217075 \\
\hline $\mathrm{C}$ & -10.14861145 & -1.19374924 & 0.02547012 \\
\hline $\mathrm{C}$ & -9.96343370 & 1.28817762 & -0.33610645 \\
\hline $\mathrm{C}$ & -7.40301027 & -2.32715588 & -1.02884005 \\
\hline $\mathrm{C}$ & -6.68042276 & -3.51364735 & -1.09747757 \\
\hline $\mathrm{C}$ & -5.68382404 & -3.81354167 & -0.15359605 \\
\hline $\mathrm{C}$ & -5.44734563 & -2.87428279 & 0.86474640 \\
\hline $\mathrm{C}$ & -6.16018770 & -1.68193166 & 0.92537507 \\
\hline $\mathrm{C}$ & -10.02186895 & -2.11588941 & 1.07368131 \\
\hline $\mathrm{C}$ & -10.87411266 & -3.21423264 & 1.19615344 \\
\hline $\mathrm{C}$ & -11.88755758 & -3.41399570 & 0.25150419 \\
\hline $\mathrm{C}$ & -12.04001360 & -2.49653825 & -0.79919288 \\
\hline $\mathrm{C}$ & -11.19207442 & -1.40335289 & -0.89886037 \\
\hline $\mathrm{C}$ & -11.11990343 & 1.63821087 & 0.38968683 \\
\hline $\mathrm{C}$ & -11.79795067 & 2.82349416 & 0.14500119 \\
\hline $\mathrm{C}$ & -11.35243169 & 3.69691659 & -0.85875052 \\
\hline $\mathrm{C}$ & -10.21976314 & 3.36024420 & -1.60900098 \\
\hline $\mathrm{C}$ & -9.54256729 & 2.16980625 & -1.34142698 \\
\hline $\mathrm{C}$ & -4.73357726 & 4.77104518 & 0.60386176 \\
\hline $\mathrm{C}$ & -4.87123638 & -5.05063109 & -0.25469110 \\
\hline $\mathrm{C}$ & -5.46789337 & 5.94379787 & 0.85551339 \\
\hline $\mathrm{C}$ & -4.83896087 & 7.18445276 & 0.89661384 \\
\hline $\mathrm{C}$ & -3.46911182 & 7.28138084 & 0.67988937 \\
\hline $\mathrm{C}$ & -2.70417048 & 6.13036412 & 0.41066598 \\
\hline $\mathrm{C}$ & -3.34881544 & 4.88204625 & 0.38813965 \\
\hline $\mathrm{C}$ & -3.50846443 & -5.01478111 & 0.06601374 \\
\hline $\mathrm{C}$ & -2.68529601 & -6.14608942 & -0.04650133 \\
\hline $\mathrm{C}$ & -3.25166494 & -7.34562105 & -0.50811630 \\
\hline $\mathrm{C}$ & -4.60636635 & -7.39558485 & -0.83447065 \\
\hline $\mathrm{C}$ & -5.41369609 & -6.26541857 & -0.70842918 \\
\hline $\mathrm{C}$ & 6.68042292 & -3.51364758 & -1.09747669 \\
\hline $\mathrm{C}$ & 5.68382420 & -3.81354184 & -0.15359519 \\
\hline $\mathrm{C}$ & 5.44734573 & -2.87428290 & 0.86474718 \\
\hline $\mathrm{C}$ & 6.16018776 & -1.68193175 & 0.92537578 \\
\hline $\mathrm{C}$ & 7.16291435 & -1.38485324 & -0.01366396 \\
\hline $\mathrm{C}$ & 7.40301038 & -2.32715609 & -1.02883925 \\
\hline $\mathrm{C}$ & 7.89571668 & -0.08653161 & 0.03760395 \\
\hline $\mathrm{C}$ & 7.03688158 & 1.12214858 & 0.22323846 \\
\hline $\mathrm{C}$ & 9.25762651 & -0.00184507 & -0.08217037 \\
\hline
\end{tabular}




\begin{tabular}{|c|c|c|c|}
\hline $\mathrm{C}$ & 9.96343349 & 1.28817762 & -0.33610643 \\
\hline $\mathrm{C}$ & 10.14861155 & -1.19374919 & 0.02547059 \\
\hline $\mathrm{C}$ & 5.88486408 & 1.33213375 & -0.55539764 \\
\hline $\mathrm{C}$ & 5.11980916 & 2.48887122 & -0.41842625 \\
\hline $\mathrm{C}$ & 5.45742116 & 3.47550997 & 0.52302549 \\
\hline $\mathrm{C}$ & 6.56554325 & 3.22688880 & 1.35284322 \\
\hline $\mathrm{C}$ & 7.33823114 & 2.08172502 & 1.20454787 \\
\hline $\mathrm{C}$ & 9.54256655 & 2.16980618 & -1.34142681 \\
\hline $\mathrm{C}$ & 10.21976213 & 3.36024420 & -1.60900112 \\
\hline $\mathrm{C}$ & 11.35243100 & 3.69691673 & -0.85875114 \\
\hline $\mathrm{C}$ & 11.79795051 & 2.82349440 & 0.14500041 \\
\hline $\mathrm{C}$ & 11.11990348 & 1.63821104 & 0.38968638 \\
\hline $\mathrm{C}$ & 10.02186926 & -2.11588920 & 1.07368195 \\
\hline $\mathrm{C}$ & 10.87411303 & -3.21423237 & 1.19615410 \\
\hline $\mathrm{C}$ & 11.88755779 & -3.41399554 & 0.25150470 \\
\hline $\mathrm{C}$ & 12.04001360 & -2.49653825 & -0.79919254 \\
\hline $\mathrm{C}$ & 11.19207432 & -1.40335295 & -0.89886006 \\
\hline $\mathrm{C}$ & 4.87123654 & -5.05063128 & -0.25469023 \\
\hline $\mathrm{C}$ & 4.73357743 & 4.77104541 & 0.60386018 \\
\hline $\mathrm{C}$ & 5.41369630 & -6.26541878 & -0.70842812 \\
\hline $\mathrm{C}$ & 4.60636658 & -7.39558507 & -0.83446962 \\
\hline $\mathrm{C}$ & 3.25166510 & -7.34562126 & -0.50811552 \\
\hline $\mathrm{C}$ & 2.68529612 & -6.14608958 & -0.04650075 \\
\hline $\mathrm{C}$ & 3.50846453 & -5.01478125 & 0.06601439 \\
\hline $\mathrm{C}$ & 3.34881551 & 4.88204644 & 0.38813873 \\
\hline $\mathrm{C}$ & 2.70417068 & 6.13036439 & 0.41066440 \\
\hline $\mathrm{C}$ & 3.46911233 & 7.28138126 & 0.67988641 \\
\hline $\mathrm{C}$ & 4.83896148 & 7.18445319 & 0.89661019 \\
\hline $\mathrm{C}$ & 5.46789384 & 5.94379824 & 0.85551043 \\
\hline $\mathrm{C}$ & -0.70903606 & -5.20963926 & 1.26408299 \\
\hline $\mathrm{C}$ & 0.70903592 & -5.20963930 & 1.26408313 \\
\hline $\mathrm{C}$ & 1.26350110 & -6.01043843 & 0.29182737 \\
\hline S & 0.00000010 & -6.79149124 & -0.63869573 \\
\hline $\mathrm{C}$ & -1.26350107 & -6.01043832 & 0.29182708 \\
\hline $\mathrm{C}$ & -1.26696918 & 6.31676845 & 0.14709932 \\
\hline S & 0.00000029 & 5.29250440 & 0.80423103 \\
\hline $\mathrm{C}$ & 1.26696924 & 6.31676866 & 0.14709857 \\
\hline $\mathrm{C}$ & 0.70853752 & 7.36617197 & -0.54614700 \\
\hline $\mathrm{C}$ & -0.70853803 & 7.36617186 & -0.54614656 \\
\hline $\mathrm{O}$ & -12.08988416 & 4.83285700 & -1.02946282 \\
\hline $\mathrm{C}$ & -11.68948844 & 5.74645603 & -2.03805722 \\
\hline $\mathrm{O}$ & -12.77423571 & -4.45147720 & 0.26793653 \\
\hline $\mathrm{C}$ & -12.66871856 & -5.40749869 & 1.31057667 \\
\hline $\mathrm{O}$ & 12.77423592 & -4.45147699 & 0.2679370 \\
\hline
\end{tabular}




\begin{tabular}{|c|c|c|c|}
\hline $\mathrm{C}$ & 12.66871903 & -5.40749826 & 1.31057739 \\
\hline $\mathrm{O}$ & 12.08988326 & 4.83285726 & -1.02946374 \\
\hline $\mathrm{C}$ & 11.68948717 & 5.74645608 & -2.03805817 \\
\hline $\mathrm{H}$ & -4.27481281 & 2.64848128 & -1.08297419 \\
\hline $\mathrm{H}$ & -6.82098954 & 3.94022065 & 2.13120504 \\
\hline $\mathrm{H}$ & -8.19641858 & 1.92679892 & 1.85103956 \\
\hline $\mathrm{H}$ & -5.60793118 & 0.59543902 & -1.30456701 \\
\hline $\mathrm{H}$ & -8.15546005 & -2.11422582 & -1.78155742 \\
\hline $\mathrm{H}$ & -6.86261785 & -4.19995680 & -1.91995797 \\
\hline $\mathrm{H}$ & -4.70282318 & -3.08769382 & 1.62665280 \\
\hline $\mathrm{H}$ & -5.94707305 & -0.97087323 & 1.71872137 \\
\hline $\mathrm{H}$ & -9.24146818 & -1.97261068 & 1.81450494 \\
\hline $\mathrm{H}$ & -10.74133812 & -3.89653502 & 2.02816435 \\
\hline $\mathrm{H}$ & -12.83417217 & -2.66135856 & -1.52091545 \\
\hline $\mathrm{H}$ & -11.33119507 & -0.69684993 & -1.71214296 \\
\hline $\mathrm{H}$ & -11.48537975 & 0.96803509 & 1.16254105 \\
\hline $\mathrm{H}$ & -12.68121979 & 3.09518826 & 0.71491122 \\
\hline $\mathrm{H}$ & -9.85986646 & 4.00684738 & -2.40124890 \\
\hline $\mathrm{H}$ & -8.66702734 & 1.92093331 & -1.93278889 \\
\hline $\mathrm{H}$ & -6.54336913 & 5.88120514 & 0.98870061 \\
\hline $\mathrm{H}$ & -5.42014851 & 8.08082468 & 1.09667543 \\
\hline $\mathrm{H}$ & -2.97437195 & 8.24574107 & 0.73615021 \\
\hline $\mathrm{H}$ & -2.76501175 & 3.98673139 & 0.19080175 \\
\hline $\mathrm{H}$ & -3.05708533 & -4.07031299 & 0.35090842 \\
\hline $\mathrm{H}$ & -2.63606877 & -8.23687868 & -0.59458421 \\
\hline $\mathrm{H}$ & -5.04052811 & -8.33023443 & -1.17968433 \\
\hline $\mathrm{H}$ & -6.47136816 & -6.32672894 & -0.94846136 \\
\hline $\mathrm{H}$ & 6.86261806 & -4.19995710 & -1.91995703 \\
\hline $\mathrm{H}$ & 4.70282323 & -3.08769388 & 1.62665357 \\
\hline $\mathrm{H}$ & 5.94707300 & -0.97087325 & 1.71872202 \\
\hline $\mathrm{H}$ & 8.15546021 & -2.11422606 & -1.78155661 \\
\hline $\mathrm{H}$ & 5.60793060 & 0.59543796 & -1.30456606 \\
\hline $\mathrm{H}$ & 4.27481229 & 2.64848034 & -1.08297411 \\
\hline $\mathrm{H}$ & 6.82099012 & 3.94022187 & 2.13120339 \\
\hline $\mathrm{H}$ & 8.19641900 & 1.92679991 & 1.85103885 \\
\hline $\mathrm{H}$ & 8.66702638 & 1.92093315 & -1.93278831 \\
\hline $\mathrm{H}$ & 9.85986509 & 4.00684733 & -2.40124889 \\
\hline $\mathrm{H}$ & 12.68121979 & 3.09518862 & 0.71491009 \\
\hline $\mathrm{H}$ & 11.48538017 & 0.96803535 & 1.16254052 \\
\hline $\mathrm{H}$ & 9.24146861 & -1.97261040 & 1.81450569 \\
\hline $\mathrm{H}$ & 10.74133865 & -3.89653464 & 2.02816514 \\
\hline $\mathrm{H}$ & 12.83417202 & -2.66135865 & -1.52091523 \\
\hline $\mathrm{H}$ & 11.33119480 & -0.69685012 & -1.71214279 \\
\hline $\mathrm{H}$ & 6.47136843 & -6.32672920 & -0.94846011 \\
\hline
\end{tabular}




$\begin{array}{lrrc}\mathrm{H} & 5.04052838 & -8.33023470 & -1.17968314 \\ \mathrm{H} & 2.63606893 & -8.23687884 & -0.59458346 \\ \mathrm{H} & 3.05708539 & -4.07031310 & 0.35090892 \\ \mathrm{H} & 2.76501166 & 3.98673146 & 0.19080183 \\ \mathrm{H} & 2.97437262 & 8.24574160 & 0.73614675 \\ \mathrm{H} & 5.42014936 & 8.08082526 & 1.09667069 \\ \mathrm{H} & 6.54336966 & 5.88120546 & 0.98869716 \\ \mathrm{H} & -1.31067595 & -4.64815110 & 1.97070959 \\ \mathrm{H} & 1.31067569 & -4.64815115 & 1.97070986 \\ \mathrm{H} & 1.30994773 & 8.11082702 & -1.05641710 \\ \mathrm{H} & -1.30994867 & 8.11082686 & -1.05641624 \\ \mathrm{H} & -12.40699580 & 6.56824618 & -1.99980584 \\ \mathrm{H} & -10.68018989 & 6.13719903 & -1.85231936 \\ \mathrm{H} & -11.71853461 & 5.28622584 & -3.03463714 \\ \mathrm{H} & -13.46018743 & -6.13707451 & 1.12916842 \\ \mathrm{H} & -11.69585725 & -5.91649206 & 1.29536928 \\ \mathrm{H} & -12.81862680 & -4.94828293 & 2.29674881 \\ \mathrm{H} & 13.46018796 & -6.13707414 & 1.12916911 \\ \mathrm{H} & 12.81862743 & -4.94828235 & 2.29674941 \\ \mathrm{H} & 11.69585778 & -5.91649169 & 1.29537028 \\ \mathrm{H} & 12.40699448 & 6.56824628 & -1.99980714 \\ \mathrm{H} & 11.71853308 & 5.28622572 & -3.03463802 \\ \mathrm{H} & 10.68018867 & 6.13719903 & -1.85232008\end{array}$

\section{Reference:}

[1] Gaussian 09, Revision C.01, M. J. Frisch, G. W. Trucks, H. B. Schlegel, G. E. Scuseria, M. A. Robb, J. R. Cheeseman, G. Scalmani, V. Barone, B. Mennucci, G. A. Petersson, H. Nakatsuji, M. Caricato, X. Li, H. P. Hratchian, A. F. Izmaylov, J. Bloino, G. Zheng, J. L. Sonnenberg, M. Hada, M. Ehara, K. Toyota, R. Fukuda, J. Hasegawa, M. Ishida, T. Nakajima, Y. Honda, O. Kitao, H. Nakai, T. Vreven, J. A. Montgomery, Jr., J. E. Peralta, F. Ogliaro, M. Bearpark, J. J. Heyd, E. Brothers, K. N. Kudin, V. N. Staroverov, T. Keith, R. Kobayashi, J. Normand, K. Raghavachari, A. Rendell, J. C. Burant, S. S. Iyengar, J. Tomasi, M. Cossi, N. Rega, J. M. Millam, M. Klene, J. E. Knox, J. B. Cross, V. Bakken, C. Adamo, J. Jaramillo, R. Gomperts, R. E. Stratmann, O. Yazyev, A. J. Austin, R. Cammi, C. Pomelli, J. W. Ochterski, R. L. Martin, K. Morokuma, V. G. Zakrzewski, G. A. Voth, P. Salvador, J. J. Dannenberg, S. Dapprich, A. D. Daniels, O. Farkas, J. B. Foresman, J. V. Ortiz, J. Cioslowski, and D. J. Fox, Gaussian, Inc., Wallingford CT, 2010. 